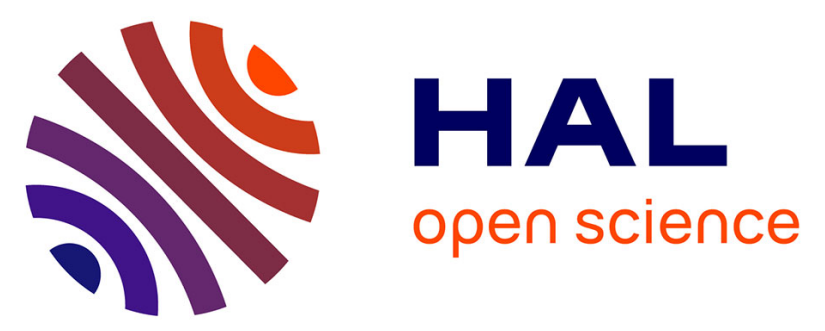

\title{
Fishing Anti-Inflammatories from Known Drugs: In Silico Repurposing, Design, Synthesis and Biological Evaluation of Bisacodyl Analogues
}

Maité Sylla-Iyarreta, Dany Siverio, Vanessa Lerari, Marta Marin, Rosa M. Giner, Liliana Vicet Muro, Yankier Rivero Guerra, Françoise Dumas, Clotilde Ferroud, Peter A. M. de Witte, et al.

\section{To cite this version:}

Maité Sylla-Iyarreta, Dany Siverio, Vanessa Lerari, Marta Marin, Rosa M. Giner, et al.. Fishing Anti-Inflammatories from Known Drugs: In Silico Repurposing, Design, Synthesis and Biological Evaluation of Bisacodyl Analogues. Current Topics in Medicinal Chemistry, 2017, 17 (25), 10.2174/1568026617666170817161953 . hal-01599101

\section{HAL Id: hal-01599101 \\ https://hal.science/hal-01599101}

Submitted on 25 May 2021

HAL is a multi-disciplinary open access archive for the deposit and dissemination of scientific research documents, whether they are published or not. The documents may come from teaching and research institutions in France or abroad, or from public or private research centers.
L'archive ouverte pluridisciplinaire HAL, est destinée au dépôt et à la diffusion de documents scientifiques de niveau recherche, publiés ou non, émanant des établissements d'enseignement et de recherche français ou étrangers, des laboratoires publics ou privés. 


\title{
Fishing Anti-Inflammatories from Known Drugs: In Silico Repurposing, Design, Synthesis and Biological Evaluation of Bisacodyl Analogues
}

\author{
Maité Sylla-Iyarreta Veitía ${ }^{a^{*}}$, Dany Siverio Mota ${ }^{\mathrm{b}, \mathrm{c}, \mathrm{d}}$, Vanessa Lerari ${ }^{2 \mathrm{a}}$, Marta Marín ${ }^{2 \mathrm{~d}}$, Rosa M. Giner ${ }^{3 \mathrm{~d}}$, \\ Liliana Vicet Muro ${ }^{2 b}$, Yankier Rivero Guerra ${ }^{3 \mathrm{~b}}$, Françoise Dumas ${ }^{\mathrm{e}}$, Clotilde Ferroud ${ }^{3 \mathrm{a}}$, \\ Peter A. M. de Witte ${ }^{2 \mathrm{c}}$, Alexander D. Crawford ${ }^{3 \mathrm{c}}$, Vicente J. Arán ${ }^{\mathrm{f}}$, and Yovani Marrero Ponce, ${ }^{\mathrm{g}, \mathrm{h}}$
}

\begin{abstract}
${ }^{a}$ Equipe de chimie moléculaire, Laboratoire Chimie Moléculaire, génie des procédés chimiques et énergétiques (CMGPCE), EA 7341-Conservatoire National des Arts et Métiers, 2 rue Conté, 75003, Paris, France; ${ }^{b}$ Unit of Computer-Aided Molecular "Biosilico" Discovery and Bioinformatic Research (CAMD-BIR Unit), Faculty of ChemistryPharmacy, Universidad Central "Marta Abreu" de Las Villas, Santa Clara, 54830, Villa Clara, Cuba; ${ }^{\circ}$ Laboratory for Molecular Biodiscovery, Department of Pharmaceutical and Pharmacological Sciences, University of Leuven, Herestraat 49, 3000 Leuven, Belgium; 'Department of Pharmacology, Faculty of Pharmacy, Universitat de València, València, Spain; ${ }^{e}$ Laboratoire BioCIS, CNRS UMR 8076, IPSIT, Faculté de Pharmacie, Université Paris Sud, Université Paris Saclay, 92296 Châtenay-Malabry Cedex, France, Instituto de Química Médica, CSIC, c/Juan de la Cierva 3, 28006 Madrid, Spain; ${ }^{g}$ Grupo de Investigación Ambiental (GIA), Programas Ambientales, Facultad de Ingenierías, Fundación Universitaria Tecnológico de Comfenalco (COMFENALCO), Cartagena de Indias, Bolivar, Colombia. ${ }^{\text {hUniversidad }}$ San Francisco de Quito (USFQ), Grupo de Medicina Molecular y Traslacional (MeM\&T), Colegio de Ciencias de la Salud (COCSA), Escuela de Medicina, Edificio de Especialidades Médicas, Av. Interoceánica Km 12 1/2 —Cumbayá. and Instituto de Simulación Computacional (ISC-USFQ), Diego de Robles y vía Interoceánica, Quito 170157, Ecuador.
\end{abstract}

\begin{abstract}
Herein is described in silico repositioning, design, synthesis, biological evaluation and structure-activity relationship (SAR) of an original class of anti-inflammatory agents based on a polyaromatic pharmacophore structurally related to bisacodyl (BSL) drug used in therapeutic as laxative. We describe the potential of TOMOCOMD-CARDD methods to find out new anti-inflammatory drug-like agents from a diverse series of compounds using the total and local atom based bilinear indices as molecular descriptors. The models obtained were validated by biological studies, identifying BSL as the first anti-inflammatory lead-like using in silico repurposing from commercially available drugs. Several biological in vitro and in vivo assays were performed in order to understand its mechanism of action. A set of analogues of BSL was prepared using low-cost synthetic procedures and further biologically investigated in zebrafish models. Compound $\mathbf{5 c}$ and $\mathbf{7 e}$ exhibited the best antiinflammatory activities and represent new promising anti-inflammatory agents for further preclinical development.
\end{abstract}

Keywords: TOMOCOMD-CARDD Software, Atom-based bilinear indices, Anti-inflammatory database, Bisacodyl, Repurposing, Diarylmethylpyridines, Anti-inflammatory assay.

\section{INTRODUCTION}

The discovery and development of new drugs is a lengthy, expensive and financially risky process. Computerassisted drug design methods and virtual screening techniques are powerful tools to reduce costs and time of research. Currently, pharmaceutical companies implement new approaches from existing drugs, in order to accelerate the discovery of interesting leads with relatively low costs and decrease risks. Drug repositioning allows the development of

*Address correspondence to this author at the Equipe de chimie moléculaire, Laboratoire Chimie Moléculaire, génie des procédés chimiques et énergétiques (CMGPCE), EA 7341- Conservatoire National des Arts et Métiers, 2 rue Conté, 75003, Paris, France; Tel: +33-1-58 8084 82;

E-mail: maite.sylla@lecnam.net new indications for existing drugs with identified pharmacokinetic profiles, known safety profile, already resolved manufacturing issues [1,2]. More and more companies are screening off the existing pharmacopoeia for repositioning candidates, and the number of repositioning success stories is growing [3].

The inflammatory process appears in presence of many phenomena and circumstances. In acute inflammation, the oedema appears following physical and chemical damage, infections by microorganisms and others, disappearing within some days after injury is repaired; however in chronic illness, the inflammation is persistent and needs a permanent treatment. At present, anti-inflammatory drugs remain one of the most used worldwide therapeutic classes. Despite their widespread use and their undeniable commercial success 
anti-inflammatory drugs show many limitations, including low efficiency, low selectivity and toxicity. It is recognized that Non-Steroidal Anti-Inflammatory Drugs (NSAIDs) cause gastrointestinal ulcers and nephrotoxicity and steroidal anti-inflammatory drugs (SAIDs) can induce osteoporosis, suppression of hypothalamus-pituitary-adrenal axis, Cushing syndrome, and reduced rate of bone growth in children [4-7]. There is therefore a medical need to discover better therapeutic alternatives for the treatment of inflammatory diseases.

One strategy to identify novel anti-inflammatory compounds is to screen libraries of diverse drug-like compounds. In this process, the identification of an suitable lead molecule (and its structural optimization) is the most perilous stage in this phase $[8,9]$. Beginning with libraries of drug compounds is an ideal starting point because substantial pharmacokinetic and toxicological data should be available for each compound series [10]. The strategy is economically interesting when compared with the cost of drug development based on de novo drug discovery and development [3]. In this sense, pharmaceutical companies make an effort to increase efficiency through novel discovery technologies have fallen short of achieving the expected results.

The most effective way to passage from target identification to the clinic is to distinguish previously approved drugs with the potential for activating or inhibiting unintended targets. This is frequently accomplished by high throughput screening (HTS) of chemicals, transcriptome matching, or in silico ligand screening [11]. The appearance of virtual screening (either ligand- or structure-based) approaches to identify a reduced number of compounds with a high potential for bioactivity to be experimentally evaluated appear both as a complementary and alternative method to primary source, HTS [12-15].

The ligand-based (LB) approaches are founded in the principle of similarity [16] and serve to model the complex phenomena of molecular recognition [17-20]. The wide structural variability of the dataset used here assures adequate generalization.

In this report, the atom-based bilinear indices implemented in the TOpological MOlecular COMputational De-signComputer-Aided Rational Drug Design (TOMOCOMDCARDD) method [21] and linear discriminant analysis (LDA) [22] have been used in order to parameterize every molecule in the database and to develop classification functions, respectively. LDA is one of the most important and simple pattern recognition methods that could be used to define which variables discriminate between two or more naturally occurring groups [23].

Here, we will investigate the potential of TOMOCOMDCARDD Molecular Descriptor (MDs) to develop antiinflammatory drug-like compounds from a varied series of molecules. Initially, we selected a wide-spectrum database of anti-inflammatory drugs. Next, the above-mentioned MDs (particularly, the total and local Non-Stochastic (NS) and Simple Stochastic (SS) bilinear indices bilinear indices) were calculated for this vast series of active/non-active compounds, and LDA was afterwards used to fit every individual classification function. Later, the models were used for the identification the starting anti-inflammatory leads compounds by using Ligand-Based Virtual Screening (LBVS) of commercially drugs. After that, some drugs were tested in in vivo and in vitro anti-inflammatory assays obtaining the best results with a drug used in therapeutics as cathartic. Finally, some analogues of that drug were synthesized and tested in Lipopolysaccharide (LPS)-enhanced leukocyte migration assay in zebrafish in order to evaluate their antiinflammatory potential and to establish Structure-Activity Relationships (SAR).

\section{MATERIALS AND METHOD}

\subsection{Computational Methods}

\subsubsection{Dataset Construction}

A total of 1213 biologically active molecules, which displayed an important structural variation, were used to integrate the general data set, from which 587 compounds have described anti-inflammatory properties and the remainder were reported for other pharmacological actions. The database of active compounds includes Glucocorticoids (GCs), cyclooxygenase-2 (COX-2) NSAIDs, and so on.

The other 626 compounds having dissimilar clinical uses such as sedative/hypnotics, anticonvulsants, antivirals, diuretics, antihelminthics, hemostatics, oral hypoglycemics, anti-hypertensives, cathartics, and anticancer were selected like "inactive" compounds through random selection, guaranteeing great structural variability as well. The classification of these organic compounds as "inactive" (non-antiinflammatory compounds) does not guarantee that some of them have unidentified anti-inflammatory activity.

All compounds were taken from the Merck Index [24] and Negwer Handbook [25] The chemical structures of all active compounds used in the database are listed in Table SI1 (See Supporting Information).

\subsubsection{Representation of Chemical Structures}

Numerous types of representations are usually used in this regard, all well-known like MDs or molecular indices. These parameters are numbers that illustrate a specific feature of the molecular structure [26-28].

In this work, a 2D TOMOCOMD-CARDD MD family, namely atom, atom-type, and total bilinear indices were used to codify the chemical structure of the molecules in the dataset. The MDs are based on the calculation of bilinear maps in $\mathfrak{R}^{n}$ in canonical basis sets [29-34]. The computation of the NS and SS bilinear indices is develop by using the $k^{\text {th }}$ "nonstochastic and stochastic graph-theoretical electronicdensity matrices" $\mathrm{M}^{k}$ and $\mathrm{S}^{k}$, correspondingly, as matrices of the mathematical forms. The theoretical scaffold of these atom-based MDs and its use to represent small-to-medium size organic chemicals, as well as QSAR and drug design studies, have been described elsewhere [29-34]. All MDs [total and local (both atom and atom-type)] NS and SS bilinear indices were calculated with TOMOCOMD-CARDD software [29].

\subsubsection{Chemometric Studies}

The STATISTICA software [22] was used to establish the LDA models. The LDA is one of the most widely used 
programs nowadays; furthermore, its use in drug discovery has been extensively outlined [35-41].

The objective of LDA, a heuristics algorithm able to differentiating among two or more categories of objects, is to find a linear function allowing one to discriminate among active and inactive compounds [23]. The LDA was chosen between several statistical methods to get classification functions, owing to its straightforwardness. The quality of the models was evaluated by examining Wilks' $\lambda$ parameter $(U$ statistic), the square Mahalanobis distance $\left(D^{2}\right)$, the Fisher ratio $(F)$, and the corresponding $p$ level $[p(F)]$, as well as the percentage of good classification (accuracy) in the training and test sets. The classification of cases was performed by means of the posterior classification probabilities.

The biological activity was coded by a dummy variable "Class". This variable designates the presence of either an active compound (Class $=1$ ) or an inactive compound (Class $=-1$ ). By using the models, one compound can then be classified as active if $\% \Delta \mathrm{P}>0$, where $\% \Delta \mathrm{P}=[\mathrm{P}$ (Active)-P (Inactive) $] x 100$. The $\mathrm{P}$ (Active) and $\mathrm{P}$ (Inactive) are the probabilities with which the equations classify a compound as active or inactive, correspondingly.

For the assessment of the obtained models, the specificity (also known as "hit rate"), the false positive rate (also known as "false alarm rate"), the sensibility and Matthews' correlation coefficient $(C)$, were calculated and examined in the training and test sets [42].

Lastly, leverage approach [43] was used to estimate the applicability domain (AD) of QSAR models. Using this method, it is possible to confirm whether a novel chemical will lie within the structural model domain. The impact on the model is measured by the leverage $h$. Therefore is, leverage used as a quantitative measure of the model AD is appropriate for evaluating the degree of extrapolation, which means a sort of compound "distance" from the model experimental space. Leverage values can be calculated for both training compounds and new compounds. In the first case, they are suitable for finding training compounds that influence model parameters to a marked extent, resulting in an unstable model. In the second case, they are useful for checking the AD of the model [44].

Finally, an ensemble classifier $C_{\mathrm{E}}$ is used in order to make predictions. The algorithm of this multi-agent classifier is presented in the supporting information (see SI6). Here, the final predicted result should be determined by a fusion approach through the following voting mechanism.

\subsection{Materials and Chemical Methods}

All reagents were obtained from commercial sources unless otherwise noted, and used as received. Heated experiments were conducted using thermostatically controlled oil baths and were performed when necessary under inert atmosphere in oven-dried glassware. All reactions were monitored by analytical thin layer chromatography (TLC) performed on aluminium sheets precoated silica gel plates $(60$ $\mathrm{F}_{254}$, Merck). TLC plates were visualized using irradiation with light at $254 \mathrm{~nm}$, ninhydrine sprays or in an iodine chamber as appropriate. Flash column chromatography (FCC) were carried out when necessary using silica gel 60 (particle size 0.040-0.063 mm, Merck).

Melting Points (Mp) were determined on a Leica VMHB system Kofler apparatus. The structure of the products prepared was checked by comparison of their NMR, IR and MS data and by the TLC behaviour. ${ }^{1} \mathrm{H}$ and ${ }^{13} \mathrm{C}$-NMR spectra were acquired on a Bruker BioSpin $\mathrm{GmbH}$ spectrometer 400 $\mathrm{MHz}$, at room temperature (RT). Chemical shifts are reported in $\delta$ units, Parts Per Million (ppm). Coupling constants $(J)$ are measured in hertz $(\mathrm{Hz})$. Splitting patterns are designed as follows: s, singlet; d, doublet; dd, doublet of doublets; ddd, doublet of doublet of doublets; m, multiplet; br, broad. Various 2D techniques and DEPT experiments were used to establish the structures and to assign the signals. For the assignments of the NMR signals, we use the conventional numbering presented in Scheme 1. GC-MS analyses were performed with an Agilent $6890 \mathrm{~N}$ instrument equipped with a $12 \mathrm{~m} \times 0.20 \mathrm{~mm}$ dimethyl polysiloxane capillary column and an Agilent 5973N MS detector-column temperature gradient $100-300{ }^{\circ} \mathrm{C}(\operatorname{method} 100): 100{ }^{\circ} \mathrm{C}(1 \mathrm{~min}) ; 100{ }^{\circ} \mathrm{C}$ to $220{ }^{\circ} \mathrm{C}\left(10{ }^{\circ} \mathrm{C} / \mathrm{min}\right) ; 200{ }^{\circ} \mathrm{C}(2 \mathrm{~min}) ;$ gradient $160-280{ }^{\circ} \mathrm{C}$ (method 160): $160{ }^{\circ} \mathrm{C}(1 \mathrm{~min}) ; 160{ }^{\circ} \mathrm{C}$ to $280{ }^{\circ} \mathrm{C}(10$ $\left.{ }^{\circ} \mathrm{C} / \mathrm{min}\right) ; 280{ }^{\circ} \mathrm{C}(2 \mathrm{~min})$; gradient $180-300{ }^{\circ} \mathrm{C}(\operatorname{method} 180)$ : $180{ }^{\circ} \mathrm{C}(1 \mathrm{~min}) ; 180{ }^{\circ} \mathrm{C}$ to $300{ }^{\circ} \mathrm{C}\left(10{ }^{\circ} \mathrm{C} / \mathrm{min}\right) ; 300{ }^{\circ} \mathrm{C}(2$ min); gradient $200-320{ }^{\circ} \mathrm{C}(\operatorname{method} 220): 200{ }^{\circ} \mathrm{C}(1 \mathrm{~min})$; $200{ }^{\circ} \mathrm{C}$ to $320{ }^{\circ} \mathrm{C}\left(10{ }^{\circ} \mathrm{C} / \mathrm{min}\right) ; 320{ }^{\circ} \mathrm{C}$ (2 min), lowresolution mass spectra (LRMS) resulting from ionization by electronic impact. Infrared spectra were recorded over the $400-4000 \mathrm{~cm}^{-1}$ range with an Agilent Technologies Cary 630 FTIR/ATR/ZnSe spectrometer. LRMS were also performed from ionization by electrospray (ESI-LRMS) on a Waters Micromass ZQ2000 (mass) spectrometer. The samples were prepared in $\mathrm{MeOH}$ or $\mathrm{MeCN}$ and the analyses were performed in $\mathrm{MeOH} / \mathrm{HCOOH} 0.05 \%$. The cone voltage $(\mathrm{CV})$ and the $\mathrm{m} / \mathrm{z}$ resulting from fragmentation processes are indicated, and sometimes assigned; the corresponding ionic abundances were reported in percentage relative to the more abundance. High-resolution mass spectra (HRMS) analyses were acquired on a Thermo Scientific LTQ Orbitrap mass spectrometer. The HPLC analyses were carried out on a normal phase column Hypersil Si (length: $150 \mathrm{~mm}$, diameter: $4.6 \mathrm{~mm}$, stationary phase: $5 \mu \mathrm{m}$ ) and a reverse phase column Hypersil ODS C18 (length: $150 \mathrm{~mm}$, diameter: 4.6 $\mathrm{mm}$, stationary phase: $5 \mu \mathrm{m}$ ) using a Water 2998 Photodiode Array Detector $(260-370 \mathrm{~nm})$ and an isocratic system of elution. The retention times $R_{t}$ are expressed in minutes in the decimal system. HPLC purity were determined on the Hypersil Si column, using heptane/AcOEt $7 / 3$ with a flow rate of $0.8 \mathrm{~mL}$ per min and UV detection at $\lambda=262 \mathrm{~nm}$, unless otherwise notified.

\section{EXPERIMENTAL}

\subsection{Chemical Synthesis}

\subsubsection{General Procedure for the Preparation of Diarylme- thylpyridines by Friedel-Crafts Reaction}

Method A: To a solution of 2-pyridinecarboxaldehyde 2 ( 1 equiv) in the corresponding aromatic compound 3 (2-6.6 equiv) was added dropwise a mixture of Triflic Acid (trifluo- 
romethanesulfonic acid, TFSA $)(0.44 \mathrm{~mL} / \mathrm{mmol})$ and trifluoroacetic acid (TFA) $(1.2 \mathrm{~mL} / \mathrm{mmol})$ at $0{ }^{\circ} \mathrm{C}$. The reaction mixture was stirred at RT (monitoring by TLC, cyclohexane $(\mathrm{Cy}) /$ EtOAc 1:1). After reaction completion, the reaction mixture was cooled then neutralized with saturated $\mathrm{Na}_{2} \mathrm{CO}_{3}$ solution ( $\mathrm{pH} 7-8)$ and extracted with dichloromethane three times. The combined organic phases were washed with water then with brine, dried over anhydrous $\mathrm{Na}_{2} \mathrm{SO}_{4}$, filtered and concentrated. The crude residue was purified by FCC on silica gel to afford the corresponding diarylmethylpyridines 4: Yields: 4a, 78\%; 4b, 72\%; 4c, 50\%; 4d, 37\%; In some cases the $o, p$-regioisomers $\mathbf{5}$ were also isolated. Yields: 5a, $4 \% ; \mathbf{5 b}, 16 \% ; \mathbf{5 c}, 13 \%, \mathbf{5 d}, 8 \%$.

Method B: To a solution of 2-pyridinecarboxaldehyde 2 (1 equiv) in the corresponding aromatic compound 3 (2 equiv) was added dropwise concentrated sulfuric acid at RT. The reaction mixture was stirred at RT (monitoring by TLC, $\mathrm{Cy} /$ EtOAc 1:1). After reaction completion the reaction mixture was cooled then neutralized with $9 \mathrm{M} \mathrm{NaOH}$ solution ( $\mathrm{pH} 7-8$ ). The yellow precipitate formed was dissolved in diethyl ether. The organic phase was dried over anhydrous $\mathrm{Na}_{2} \mathrm{SO}_{4}$, filtered and concentrated. The crude residue was purified by crystallisation or by FCC on silica gel to afford the corresponding diarylmethylpyridines 4: Yields: 4e, 34\%; 4f, $60 \%$.

\subsubsection{2-[bis(4-fluorophenyl)-methyl]pyridine 4a[45]}

From 2-pyridinecarboxaldehyde 2 (607 mg, $5.7 \mathrm{mmol})$ and fluorobenzene ( $3.13 \mathrm{~g}, 32.6 \mathrm{mmol}, 5.7$ equiv). Method A. Reaction time: 2 h. FCC: Cy/EtOAc 9/1. 4a: $R_{f}=0.37$, $\mathrm{SiO}_{2}$, (Cy/EtOAc 8:2); yellow oil, Yield: $1.25 \mathrm{~g}, 78 \%$ (and 5a $65 \mathrm{mg}$, 4\%, yellow oil). HPLC purity: 93\% $\left(R_{t}=3.86\right.$ min). IR (ATR): $v$ 3052, $3012\left({ }_{\text {Csp2-H }}\right), 2921\left(_{\text {Csp3-H }}\right), 1603$, 1588, 1504, $1471\left(v_{\mathrm{C}=\mathrm{C}}\right), 1220\left(\mathrm{v}_{\mathrm{C}-\mathrm{F}}\right), 820,805\left(\delta_{\mathrm{Csp} 2-\mathrm{H}}\right.$ p-disubst). LRMS (ESI, CV = 35): 282.26 for $\mathrm{C}_{18} \mathrm{H}_{13} \mathrm{~F}_{2} \mathrm{~N}$ $[\mathrm{M}+\mathrm{H}]^{+}$(100); HRMS: calcd. for $\mathrm{C}_{18} \mathrm{H}_{13} \mathrm{~F}_{2} \mathrm{~N} \quad[\mathrm{M}+\mathrm{H}]^{+}$ (282.10888); found (282.10927). NMR data were in agreement with those previously reported (see SI). 2',4fluorophenyl-2-methylpyridine $\quad \mathbf{5 a} \quad R_{f}=0.44, \quad \mathrm{SiO}_{2}$, (Cy/EtOAc 8:2); ${ }^{1} \mathrm{H}$ NMR $\left(\mathrm{CDCl}_{3}, 400 \mathrm{MHz}\right) \delta=5.95(\mathrm{~s}$, $\left.1 \mathrm{H}, \mathrm{H}_{1}\right) ; 6.99\left(\mathrm{dd}, 2 \mathrm{H}, J_{\text {ortho }}=J_{\mathrm{H}-\mathrm{F}}=8.7 \mathrm{~Hz}, \mathrm{H}_{4}, \mathrm{H}_{6}\right.$ ) $)$ 7.02$7.08\left(\mathrm{~m}, 3 \mathrm{H}, \mathrm{H}_{5}, \mathrm{H}_{4}, \mathrm{H}_{6}\right) ; 7.08-7.13\left(\mathrm{dm}, 1 \mathrm{H}, J_{9-10}=7.7 \mathrm{~Hz}\right.$, $\left.\mathrm{H}_{9}\right)$; 7.13-7.19 (m, 3H, $\left.\mathrm{H}_{3}, \mathrm{H}_{7}, \mathrm{H}_{3}\right)$; 7.20-7.25 (m, 1H, $\left.\mathrm{H}_{11}\right)$; $7.62\left(\mathrm{ddd}, 1 \mathrm{H}, J_{10-9}=J_{10-11}=7.7 \mathrm{~Hz}, J_{10-12}=1.8 \mathrm{~Hz}, \mathrm{H}_{10}\right)$; $8.61\left(\mathrm{~d}, 1 \mathrm{H}, J_{11-12}=4.0 \mathrm{~Hz}, \mathrm{H}_{12}\right) .{ }^{13} \mathrm{C} \mathrm{NMR}\left(\mathrm{CDCl}_{3}, 100\right.$ $\mathrm{MHz}): \delta$ ppm: $51.2\left(\mathrm{C}_{1}\right) ; 115.44\left(\mathrm{~d}, 2 \mathrm{C}, \mathrm{J}_{\mathrm{C}-\mathrm{F}}=21.3 \mathrm{~Hz}, \mathrm{C}_{4}\right.$, $\left.\mathrm{C}_{6},\right) ; 115.55\left(\mathrm{~d}, 1 \mathrm{C}, \mathrm{J}_{\mathrm{C}-\mathrm{F}}=22.0 \mathrm{~Hz}, \mathrm{C}_{6}\right) ; 121.85\left(\mathrm{C}_{11}\right)$; $123.68\left(\mathrm{C}_{9}\right) ; 124.17\left(\mathrm{~d}, 1 \mathrm{C}, \mathrm{J}_{\mathrm{C}-\mathrm{F}}=3.4 \mathrm{~Hz}, \mathrm{C}_{4}\right) ; 128.67(\mathrm{~d}$, $\left.1 \mathrm{C}, \mathrm{J}_{\mathrm{C}-\mathrm{F}}=8.11 \mathrm{~Hz}, \mathrm{C}_{5}\right) ; 128.67\left(\mathrm{~d}, 1 \mathrm{C}, \mathrm{J}_{\mathrm{C}-\mathrm{F}}=14.1 \mathrm{~Hz}, \mathrm{C}_{2}\right)$; $130.75\left(\mathrm{~d}, 1 \mathrm{C}, \mathrm{J}_{\mathrm{C}-\mathrm{F}}=3.8 \mathrm{~Hz}, \mathrm{C}_{3}\right), 130.87\left(\mathrm{~d}, 2 \mathrm{C}, \mathrm{J}_{\mathrm{C}-\mathrm{F}}=7.9\right.$, $\mathrm{J}_{\mathrm{C}-\mathrm{F}}=3.8 \mathrm{~Hz}, \mathrm{~J}_{\mathrm{C}-\mathrm{F}}=3.8 \mathrm{~Hz}, \mathrm{C}_{3}, \mathrm{C}_{7}, 136.9\left(\mathrm{C}_{10}\right) ; 137.19(\mathrm{~d}$, $\left.2 \mathrm{C}, \mathrm{J}_{\mathrm{C}-\mathrm{F}}=3.2 \mathrm{~Hz}, \mathrm{C}_{2}\right) ; 149.49\left(\mathrm{C}_{12}\right) ; 160.77\left(\mathrm{~d}, 1 \mathrm{C}, \mathrm{J}_{\mathrm{C}-\mathrm{F}}=\right.$ $\left.245.4 \mathrm{~Hz}, \mathrm{C}_{7}\right) ; 161.76\left(\mathrm{C}_{8}\right) ; 161.79\left(\mathrm{~d}, 1 \mathrm{C}, \mathrm{J}_{\mathrm{C}-\mathrm{F}}=243.9 \mathrm{~Hz}\right.$, $\mathrm{C}_{5}$ ). IR (ATR): $v$ 3055, $3010\left(_{\mathrm{Csp} 2-\mathrm{H}}\right), 2925,2960\left(_{\mathrm{Csp} 3-\mathrm{H}}\right)$, $1605,1587,1500,1478\left(v_{\mathrm{C}=\mathrm{C}}\right), 1225\left(\mathrm{v}_{\mathrm{C}-\mathrm{F}}\right)$. LRMS (ESI, $\mathrm{CV}=35): 282.26 \quad[\mathrm{M}+\mathrm{H}]^{+} \quad(100) ;$ HRMS: calcd. for $\mathrm{C}_{18} \mathrm{H}_{13} \mathrm{~F}_{2} \mathrm{~N}[\mathrm{M}+\mathrm{H}]^{+}$(282.10888); found (282.10925).

\subsubsection{2-[bis(4-chlorophenyl)-methyl]pyridine 4b[45]}

From 2-pyridinecarboxaldehyde 2 (656 mg, $6.1 \mathrm{mmol})$ and chlorobenzene (4.07 g, $36.2 \mathrm{mmol}, 5.9$ equiv). Method
A. Reaction time $24 \mathrm{~h}$. After workup, the crude product was precipitated with petroleum ether. FCC: Cy/EtOAc 95/5. 4b White solid, $\mathrm{Mp}=76-77{ }^{\circ} \mathrm{C}, R_{f}=0.41, \mathrm{SiO}_{2},(\mathrm{Cy} / \mathrm{EtOAc}$ 8/2), Yield: $1.38 \mathrm{~g}, 72 \%$ (and 5b: $310 \mathrm{mg}, 16 \%$, white solid). HPLC purity: 93\%, $\left(R_{t}=3.61 \mathrm{~min}\right)$. IR (ATR): $v$ 3083, 3065, 3047, $3004\left(v_{\mathrm{Csp} 2-\mathrm{H}}\right), 2912$ and $2851\left(\mathrm{v}_{\mathrm{Csp} 3-\mathrm{H}}\right), 1586,1561$, $1482,1463\left(v_{\mathrm{C}=\mathrm{C}}\right), 879\left(\delta_{\mathrm{Csp} 2-\mathrm{H}}\right.$ p-disubst $)$. LRMS (ESI, CV $=$ 30): 314.22 for $\mathrm{C}_{18} \mathrm{H}_{13} \mathrm{Cl}_{2} \mathrm{~N}$ [M+H] $]^{+}$(100); HRMS: calcd. for $\mathrm{C}_{18} \mathrm{H}_{13} \mathrm{Cl}_{2} \mathrm{~N}$ [M+H] $]^{+}$(314.04978); found (314.05014). NMR data were in agreement with those previously reported (see SI). 2',4-chlorophenyl-2-methylpyridine 5b $\mathrm{Mp}=66-68$ ${ }^{\circ} \mathrm{C}, R_{f}=0.51, \mathrm{SiO}_{2}$, (Cy/EtOAc 8/2); ${ }^{1} \mathrm{H}$ NMR (DMSO-d6, $400 \mathrm{MHz}) \delta=5.98\left(\mathrm{~s}, 1 \mathrm{H}, \mathrm{H}_{1}\right) ; 7.03\left(\mathrm{dd}, 1 \mathrm{H}, J_{3-4}=5.7 \mathrm{~Hz}\right.$, $\left.J_{3-5}=3.6 \mathrm{~Hz}, \mathrm{H}_{3}\right) ; 7.13\left(\mathrm{~d}, 2 \mathrm{H}, J_{\text {ortho }}=8.6 \mathrm{~Hz}, \mathrm{H}_{3}, \mathrm{H}_{7}\right.$ ); 7.17 $\left(\mathrm{dm}, 1 \mathrm{H}, J_{9-10}=7.7 \mathrm{~Hz}, \mathrm{H}_{9}\right)$; 7.24-7.29 (m, 3H, $\left.\mathrm{H}_{11}, \mathrm{H}_{3}, \mathrm{H}_{4}\right)$; $7.36\left(\mathrm{~d}, 2 \mathrm{H}, J_{\text {ortho }}=8.5 \mathrm{~Hz}, \mathrm{H}_{4}, \mathrm{H}_{6}\right) ; 7.44\left(\mathrm{dd}, 1 \mathrm{H}, J_{6-5}=7.4\right.$ $\left.\mathrm{Hz}, J_{6-4}=3.5 \mathrm{~Hz}, \mathrm{H}_{6}\right) ; 7.74\left(\mathrm{ddd}, 1 \mathrm{H}, J_{10-12}=1.8 \mathrm{~Hz}, J_{10-9}=\right.$ $\left.J_{10-11}=7.7 \mathrm{~Hz}, \mathrm{H}_{10}\right) ; 8.53\left(\mathrm{ddd}, 1 \mathrm{H}, J_{12-11}=4.7 \mathrm{~Hz}, J_{12-10}=\right.$ $\left.1.6 \mathrm{~Hz}, J_{12-9}=0.6 \mathrm{~Hz}, \mathrm{H}_{12}\right) .{ }^{13} \mathrm{C} \mathrm{NMR}\left(\mathrm{CDCl}_{3}, 100 \mathrm{MHz}\right): \delta$ $=55.3\left(\mathrm{C}_{1}\right) ; 121.8\left(\mathrm{C}_{11}\right) ; 124.1\left(\mathrm{C}_{9}\right) ; 126.9\left(\mathrm{C}_{4}\right) ; 128.3\left(\mathrm{C}_{5}\right)$; $128.8\left(\mathrm{C}_{4}, \mathrm{C}_{6}\right) ; 129.9\left(\mathrm{C}_{6}\right) ; 130.9\left(\mathrm{C}_{3}, \mathrm{C}_{7}\right) ; 131.0\left(\mathrm{C}_{3}\right)$; $132.8\left(\mathrm{C}_{5}\right) ; 134.7\left(\mathrm{C}_{7}\right) ; 136.8\left(\mathrm{C}_{10}\right) ; 140.1$ and $140.2\left(\mathrm{C}_{2}\right.$, $\left.\mathrm{C}_{2}\right) ; 149.9\left(\mathrm{C}_{12}\right) ; 161.6\left(\mathrm{C}_{8}\right)$. HPLC purity: $92 \%\left(R_{t}=3.11\right.$ min). IR (ATR): $v$ 3059, $3011\left(v_{\mathrm{Csp} 2-\mathrm{H}}\right), 2917\left(v_{\mathrm{Csp} 3-\mathrm{H}}\right), 1586$, $1571,1489,1465\left(v_{\mathrm{C}=\mathrm{C}}\right), 819\left(\delta_{\mathrm{Csp} 2-\mathrm{H}}\right.$ p-disubst $), 750\left(\delta_{\mathrm{Csp} 2-\mathrm{H}}\right.$ o-disubst). LRMS (ESI, CV = 30): 314.16 for $\mathrm{C}_{18} \mathrm{H}_{13} \mathrm{Cl}_{2} \mathrm{~N}$ $[\mathrm{M}+\mathrm{H}]^{+}$(100); HRMS: calcd. for $\mathrm{C}_{18} \mathrm{H}_{13} \mathrm{Cl}_{2} \mathrm{~N} \quad[\mathrm{M}+]^{+}$ (315.21701); found (315.21701).

\subsubsection{2-[bis(4-bromophenyl)-methyl]pyridine 4c[45]}

From 2-pyridinecarboxaldehyde $2(710 \mathrm{mg}, 6.6 \mathrm{mmol})$ and bromobenzene ( $6.85 \mathrm{~g}, 43.6 \mathrm{mmol}, 6.6$ equiv). Method A. Reaction time: $2 \mathrm{~h}$ at $50{ }^{\circ} \mathrm{C}$. FCC: Cy/EtOAc 9/1. 4c: $R_{f}=$ $0.40, \mathrm{SiO}_{2},\left(\mathrm{Cy} /\right.$ EtOAc 8/2); white solid, $\mathrm{Mp}=102-103{ }^{\circ} \mathrm{C}$, Yield: $1.33 \mathrm{~g}, 50 \%$ (and 5c, yield: $360 \mathrm{mg}, 13 \%$, yellow oil). HPLC purity: $96 \%\left(R_{t}=3.56 \mathrm{~min}\right)$. GC/MS: method $160 ; R_{t}$ $=10.50 \mathrm{~min} m / z: 403[\mathrm{M}]^{+}(47), 402[\mathrm{M}-\mathrm{H}]^{+}(63), 323[\mathrm{M}-$ $\mathrm{Br}]^{+}(25), 165\left[\mathrm{Ph}_{2} \mathrm{CH}\right]^{+}(100)$, IR (ATR): v 3077, 3045, 3000 $\left(v_{\mathrm{Csp} 2-\mathrm{H}}\right), 2916\left(\mathrm{v}_{\mathrm{Csp} 3-\mathrm{H}}\right), 1584,1561,1487,1465\left(\mathrm{v}_{\mathrm{C}=\mathrm{C}}\right), 819$ $\left(\delta_{\mathrm{Csp} 2 \mathrm{H}}\right.$ p-disubst $)$. LRMS (ESI, $\left.\mathrm{CV}=35\right): 404.12$ for $\mathrm{C}_{18} \mathrm{H}_{13} \mathrm{Br}_{2} \mathrm{~N}$ [M+H] ${ }^{+}$(100); HRMS: calcd. for $\mathrm{C}_{18} \mathrm{H}_{13} \mathrm{Br}_{2} \mathrm{~N}$ $[\mathrm{M}+\mathrm{H}]^{+}$(403.94670); found (403.94729). NMR data were in agreement with those previously reported (see SI). 2',4bromophenyl-2-methylpyridine $5 \mathrm{c} \quad R_{f}=0.52, \quad \mathrm{SiO}_{2}$, (Cy/EtOAc 8/2); ${ }^{1} \mathrm{H}$ NMR $\left(\mathrm{CDCl}_{3}, 400 \mathrm{MHz}\right) \delta=6.07(\mathrm{~s}$, $\left.1 \mathrm{H}, \mathrm{H}_{1}\right) ; 7.01\left(\mathrm{~d}, 2 \mathrm{H}, J_{\text {ortho }}=8.3 \mathrm{~Hz}, \mathrm{H}_{3}, \mathrm{H}_{7}\right) ; 7.00-7.03(\mathrm{~m}$, $\left.1 \mathrm{H}, \mathrm{H}_{3}\right) ; 7.06\left(\mathrm{dm}, 1 \mathrm{H}, J_{9-10}=7.9 \mathrm{~Hz}, \mathrm{H}_{9}\right) ; 7.11(\mathrm{ddd}, 1 \mathrm{H}$, $\left.J_{5-3}=1.7 \mathrm{~Hz}, J_{5-4}=J_{5-6}=7.7 \mathrm{~Hz}, \mathrm{H}_{5}\right) ; 7.19\left(\mathrm{ddd}, 1 \mathrm{H}, J_{11-10}=\right.$ $\left.7.9 \mathrm{~Hz}, J_{11-12}=4.9 \mathrm{~Hz}, J_{11-9}=1.1 \mathrm{~Hz}, \mathrm{H}_{11}\right) ; 7.23\left(\mathrm{ddd}, 1 \mathrm{H}, J_{4-}\right.$ $\left.{ }_{3}=J_{4-5}=7.6 \mathrm{~Hz}, J_{4-6}=1.4 \mathrm{~Hz}, \mathrm{H}_{4}\right) ; 7.43\left(\mathrm{~d}, 2 \mathrm{H}, J_{\text {ortho }}=8.5\right.$ $\left.\mathrm{Hz}, \mathrm{H}_{4}, \mathrm{H}_{6}\right) ; 7.59\left(\mathrm{dd}, 1 \mathrm{H}, J_{6-5}=8.0 \mathrm{~Hz}, J_{6-4}=1.3 \mathrm{~Hz}, \mathrm{H}_{6}\right)$; $7.65\left(\mathrm{ddd}, 1 \mathrm{H}, J_{10-9}=J_{10-11}=7.7 \mathrm{~Hz}, J_{10-12}=1.8 \mathrm{~Hz}, \mathrm{H}_{10}\right)$; $8.62\left(\mathrm{ddd}, 1 \mathrm{H}, J_{12-11}=4.9 \mathrm{~Hz}, J_{12-10}=1.8 \mathrm{~Hz}, J_{12-9}=0.9 \mathrm{~Hz}\right.$, $\left.\mathrm{H}_{12}\right) .{ }^{13} \mathrm{C} \mathrm{NMR}\left(\mathrm{CDCl}_{3}, 100 \mathrm{MHz}\right): \delta=57.6\left(\mathrm{C}_{1}\right) ; 121.0$ $\left(\mathrm{C}_{5},\right) ; 122.0\left(\mathrm{C}_{11}\right) ; 124.3\left(\mathrm{C}_{9}\right) ; 125.6\left(\mathrm{C}_{7}\right) ; 127.6\left(\mathrm{C}_{4}\right) ; 128.6$ $\left(\mathrm{C}_{5}\right) ; 131.3\left(\mathrm{C}_{3}\right) ; 131.4\left(\mathrm{C}_{3}, \mathrm{C}_{7}\right) ; 131.8\left(\mathrm{C}_{4}, \mathrm{C}_{6}\right) ; 133.4\left(\mathrm{C}_{6}\right)$; $137.1\left(\mathrm{C}_{10}\right) ; 140.6\left(\mathrm{C}_{2}\right) ; 141.5\left(\mathrm{C}_{2}\right) ; 149.5\left(\mathrm{C}_{12}\right) ; 161.3\left(\mathrm{C}_{8}\right)$. HPLC purity: $92 \%\left(R_{t}=3.86 \mathrm{~min}\right)$. GC/MS: method $160 ; R_{t}$ $=10.50 \mathrm{~min} \mathrm{~m} / z: 403[\mathrm{M}]^{+}(47), 402[\mathrm{M}-\mathrm{H}]^{+}(63), 323[\mathrm{M}-$ $\mathrm{Br}]^{+}(25), 165\left[\mathrm{Ph}_{2} \mathrm{CH}\right]^{+}(100) .(\mathrm{ATR}): v 3055,3008\left(\mathrm{v}_{\mathrm{Csp} 2-\mathrm{H}}\right)$, $2960,2923\left(v_{\mathrm{Csp} 3-\mathrm{H}}\right), 1585,1574,1487,1463\left(\mathrm{v}_{\mathrm{C}=\mathrm{C}}\right), 809$ 
$\left(\delta_{\text {Csp2-H p-disubst }}\right), 778\left(\delta_{\text {Csp2-H o-disubst }}\right)$. LRMS (ESI, CV=30): $404.12[\mathrm{M}+\mathrm{H}]^{+}$(100); HRMS: calcd. for $\mathrm{C}_{18} \mathrm{H}_{13} \mathrm{Br}_{2} \mathrm{~N}$ $[\mathrm{M}+\mathrm{H}]^{+}$(403.94670); found (403.94727).

\subsubsection{2-[bis(4-iodophenyl)-methyl]pyridine 4d}

From 2-pyridinecarboxaldehyde 2 (650 mg, $6.1 \mathrm{mmol}$ ) and iodobenzene (4.96 g, $24.3 \mathrm{mmol}, 4$ equiv). Method A, Reaction time: $4 \mathrm{~h}$. FCC (Cy/EtOAc 95/5) 4d white solid, $\mathrm{Mp}=100-101{ }^{\circ} \mathrm{C}$; Yield: $1.11 \mathrm{~g}, 37 \%$ (and 5d Yield: 250 $\mathrm{mg}, 8 \%) ., \quad R_{f}=0.49, \mathrm{SiO}_{2},(\mathrm{Cy} /$ EtOAc $8 / 2) ;{ }^{1} \mathrm{H} \mathrm{NMR}$ $\left(\mathrm{CDCl}_{3}, 400 \mathrm{MHz}\right) \delta=5.58\left(\mathrm{~s}, 1 \mathrm{H}, \mathrm{H}_{1}\right) ; 6.90\left(\mathrm{~d}, 4 \mathrm{H}, J_{\text {ortho }}=\right.$ $8.2 \mathrm{~Hz}, \mathrm{H}_{3}, \mathrm{H}_{3}, \mathrm{H}_{7}, \mathrm{H}_{7}$ ); 7.09 (dm, $\left.1 \mathrm{H}, J_{9-10}=7.7 \mathrm{~Hz}, \mathrm{H}_{9}\right)$; $7.18\left(\mathrm{ddd}, 1 \mathrm{H}, J_{11-10}=7.7 \mathrm{~Hz}, J_{11-12}=4.9 \mathrm{~Hz}, J_{11-9}=1.1 \mathrm{~Hz}\right.$, $\left.\mathrm{H}_{11}\right) ; 7.62\left(\mathrm{~d}, 4 \mathrm{H}, J_{\text {ortho }}=8.2 \mathrm{~Hz}, \mathrm{H}_{4}, \mathrm{H}_{4}, \mathrm{H}_{6}, \mathrm{H}_{6}\right.$ ) $; 7.64$ (ddd, $\left.1 \mathrm{H}, J_{10-9}=J_{10-11}=7.7 \mathrm{~Hz}, J_{10-12}=1.8 \mathrm{~Hz}, \mathrm{H}_{10}\right) ; 8.59(\mathrm{ddd}$, $\left.1 \mathrm{H}, J_{12-11}=4.9 \mathrm{~Hz}, J_{12-10}=1.8 \mathrm{~Hz}, J_{12-9}=0.8 \mathrm{~Hz}, \mathrm{H}_{12}\right) .{ }^{13} \mathrm{C}$ $\operatorname{NMR}\left(\mathrm{CDCl}_{3}, 100 \mathrm{MHz}\right): \delta=58.1\left(\mathrm{C}_{1}\right) ; 92.6\left(\mathrm{C}_{5}\right.$ and $\left.\overline{\mathrm{C}}_{5},\right)$; $122.1\left(\mathrm{C}_{11}\right) ; 123.9\left(\mathrm{C}_{9}\right) ; 131.4\left(\mathrm{C}_{3}, \mathrm{C}_{3}, \mathrm{C}_{7}, \mathrm{C}_{7}\right) ; 137.2\left(\mathrm{C}_{10}\right)$; $137.7\left(\mathrm{C}_{4}, \mathrm{C}_{4}, \mathrm{C}_{6}, \mathrm{C}_{6}\right) ; 141.8\left(\mathrm{C}_{2}, \mathrm{C}_{2}\right) ; 149.5\left(\mathrm{C}_{12}\right) ; 161.8$ $\left(\mathrm{C}_{8}\right)$. HPLC purity: $96 \%,\left(R_{t}=2.99 \mathrm{~min}\right)$. GC/MS: method 200; $R_{t}=8.41 \mathrm{~min} \mathrm{~m} / \mathrm{z}: 497[\mathrm{M}]^{+}(88), 369\left[\mathrm{C}_{18} \mathrm{H}_{13} \mathrm{NI}\right]^{+}(25)$, $242\left[\mathrm{C}_{18} \mathrm{H}_{13} \mathrm{~N}\right]^{+}(88), 165\left[\mathrm{Ph}_{2} \mathrm{CH}\right]^{+}(100)$. IR (ATR): v 3061, $3042,3003\left(v_{\mathrm{Csp} 2-\mathrm{H}}\right), 2919\left(\mathrm{v}_{\mathrm{Csp} 3-\mathrm{H}}\right), 1585,1565,1475,1462$ $\left(v_{\mathrm{C}=\mathrm{C}}\right), 814\left(\delta_{\mathrm{Csp} 2-\mathrm{H} \text { p-disubst }}\right)$. LRMS (ESI, CV $\left.=35\right): 498.08$ $[\mathrm{M}+\mathrm{H}]^{+}$(100); HRMS: calcd. for $\mathrm{C}_{18} \mathrm{H}_{13} \mathrm{I}_{2} \mathrm{~N} \quad[\mathrm{M}+\mathrm{H}]^{+}$ (497.92101); found (497.92080). 2',4-iodophenyl-2methylpyridine $5 \mathbf{d}$ white solid, $\mathrm{Mp}=102-104{ }^{\circ} \mathrm{C}, R_{f}=0.56$, $\mathrm{SiO}_{2},\left(\mathrm{Cy} /\right.$ EtOAc 8/2); ${ }^{1} \mathrm{H}$ NMR $\left(\mathrm{CDCl}_{3}, 400 \mathrm{MHz}\right) \delta=5.95$ $\left(\mathrm{s}, 1 \mathrm{H}, \mathrm{H}_{1}\right) ; 6.86\left(\mathrm{~d}, 2 \mathrm{H}, J_{\text {ortho }}=8.2 \mathrm{~Hz}, \mathrm{H}_{3}, \mathrm{H}_{7}\right.$, ); 6.94 (ddd, $\left.1 \mathrm{H}, J_{5-3}=1.7 \mathrm{~Hz}, J_{5-4}=J_{5-6}=7.6 \mathrm{~Hz}, \mathrm{H}_{5}\right) ; 6.99\left(\mathrm{dd}, 1 \mathrm{H}, J_{3-5}=\right.$ $\left.1.7 \mathrm{~Hz}, J_{3-4}=7.8 \mathrm{~Hz}, \mathrm{H}_{3}\right) ; 7.03-7.07\left(\mathrm{dm}, 1 \mathrm{H}, J_{9-10}=7.7 \mathrm{~Hz}\right.$, $\left.\mathrm{H}_{9}\right) ; 7.19\left(\mathrm{ddd}, 1 \mathrm{H}, J_{11-10}=7.7 \mathrm{~Hz}, J_{11-12}=4.9 \mathrm{~Hz}, J_{11-9}=1.0\right.$ $\mathrm{Hz}, \mathrm{H}_{11}$ ); 7.27 (ddd, $1 \mathrm{H}, J_{4-3}=J_{4-5}=8.8 \mathrm{~Hz}, J_{4-6}=1.3 \mathrm{~Hz}$, $\left.\mathrm{H}_{4}\right) ; 7.60\left(\mathrm{~d}, 2 \mathrm{H}, J_{\text {ortho }}=8.4 \mathrm{~Hz}, \mathrm{H}_{4}, \mathrm{H}_{6}\right) ; 7.65(\mathrm{ddd}, 1 \mathrm{H}$, $\left.J_{10-9}=J_{10-11}=7.7 \mathrm{~Hz}, J_{10-12}=1.8 \mathrm{~Hz}, \mathrm{H}_{10}\right) ; 7.88\left(\mathrm{dd}, 1 \mathrm{H}, J_{6-5}\right.$ $\left.=7.8 \mathrm{~Hz}, J_{6-4}=1.3 \mathrm{~Hz}, \mathrm{H}_{6}\right) ; 8.62\left(\mathrm{ddd}, 1 \mathrm{H}, J_{12-11}=4.9 \mathrm{~Hz}\right.$, $\left.J_{12-10}=1.8 \mathrm{~Hz}, J_{12-9}=0.8 \mathrm{~Hz}, \mathrm{H}_{12}\right) .{ }^{13} \mathrm{C} \mathrm{NMR}\left(\mathrm{CDCl}_{3}, 100\right.$ $\mathrm{MHz}): \delta=62.3\left(\mathrm{C}_{1}\right) ; 92.6\left(\mathrm{C}_{5},\right) ; 102.5\left(\mathrm{C}_{7}\right) ; 122.0\left(\mathrm{C}_{11}\right)$; $124.6\left(\mathrm{C}_{9}\right) ; 128.4\left(\mathrm{C}_{4}\right) ; 128.8\left(\mathrm{C}_{5}\right) ; 130.9\left(\mathrm{C}_{3}\right) ; 131.8\left(\mathrm{C}_{3}\right.$, $\left.\mathrm{C}_{7}\right)$; $137.2\left(\mathrm{C}_{10}\right) ; 137.7\left(\mathrm{C} 4, \mathrm{C}_{6}\right) ; 140.2\left(\mathrm{C}_{6}\right) ; 141.4\left(\mathrm{C}_{2}\right)$; $144.5\left(\mathrm{C}_{2}\right) ; 149.4\left(\mathrm{C}_{12}\right) ; 161.3\left(\mathrm{C}_{8}\right)$. HPLC purity: $99 \%,\left(R_{t}=\right.$ $3.54 \mathrm{~min})$. GC/MS: method 200; $R_{t}=8.41 \mathrm{~min}, \mathrm{~m} / \mathrm{z}: 497$ $[\mathrm{M}]^{+}(88), 369\left[\mathrm{C}_{18} \mathrm{H}_{13} \mathrm{NI}\right]^{+}(25), 242\left[\mathrm{C}_{18} \mathrm{H}_{13} \mathrm{~N}\right]^{+}$(88), 165 $\left[\mathrm{Ph}_{2} \mathrm{CH}\right]^{+}(100)$. IR(ATR): v 3071, 3047, $3006\left(\mathrm{v}_{\mathrm{Csp} 2-\mathrm{H}}\right), 2923$ $\left(v_{\mathrm{Csp} 3-\mathrm{H}}\right), 1583,1565,1479,1462\left(\mathrm{v}_{\mathrm{C}=\mathrm{C}}\right), 1003\left(\delta_{\mathrm{Csp} 2-\mathrm{H}}\right.$ p-disubst $), 790\left(\delta_{\text {Csp2-H o-disubst }}\right)$. LRMS (ESI, CV $\left.=30\right): 498.08$ for $\mathrm{C}_{18} \mathrm{H}_{13} \mathrm{I}_{2} \mathrm{~N}[\mathrm{M}+\mathrm{H}]^{+}$(100); HRMS: calcd. for $\mathrm{C}_{18} \mathrm{H}_{13} \mathrm{I}_{2} \mathrm{~N}$ $[\mathrm{M}+\mathrm{H}]^{+}$(497.92101); found (497.92081).

\subsubsection{2-[bis(4-methylphenyl)-methyl]pyridine 4e[45]}

From 2-pyridinecarboxaldehyde 2 (1.57 g, $14.7 \mathrm{mmol})$ and toluene (2.70 g, $29.3 \mathrm{mmol}, 2$ equiv) Method B, reaction $4 \mathrm{~h}$. FCC (Cy/EtOAc 8/2 to 1/1). 4e yellow oil, Yield: $1.3 \mathrm{~g}$, $32 \% . R_{f}=0.50, \mathrm{SiO}_{2},(\mathrm{Cy} / \mathrm{EtOAc} 8 / 2)$; HPLC purity: $94 \%$ $\left(R_{t}=3.39 \mathrm{~min}\right) . \mathrm{GC} / \mathrm{MS}:$ method $160 ; R_{t}=7.23 \mathrm{~min} \mathrm{~m} / \mathrm{z}: 273$ $[\mathrm{M}]^{+}(83), 272[\mathrm{M}-\mathrm{H}]^{+}(100), 195\left[\left(\mathrm{CH}_{3} \mathrm{Ph}\right)_{2} \mathrm{CH}\right]^{+}(15), 180$ $\left[\mathrm{C}_{14} \mathrm{H}_{12}\right]^{+}$(33), $165\left[\mathrm{C}_{13} \mathrm{H}_{9}\right]^{+}$(30), IR (ATR): v 3048, 3004 $\left(v_{\mathrm{Csp} 2-\mathrm{H}}\right), 2920,2859\left(\mathrm{v}_{\mathrm{Csp} 3-\mathrm{H}}\right), 1587,1569,1511,1462$ $\left(v_{\mathrm{C}=\mathrm{C}}\right), 808\left(\delta_{\mathrm{Csp2-H}}\right.$ p-disubst $)$. HRMS: calcd. for $\mathrm{C}_{20} \mathrm{H}_{19} \mathrm{~N}$ $[\mathrm{M}+\mathrm{H}]^{+}$(274.38113); found (274.15935). NMR data were in agreement with those previously reported (see SI).

\subsubsection{2-[bis(4- tert-butylphenyl)-methyl]pyridine 4f[46]}

From 2-pyridinecarboxaldehyde 2 (1,18 g, $11.02 \mathrm{mmol})$ and tert-butylbenzene ( $3 \mathrm{~g}, 22.4 \mathrm{mmol}, 2$ equiv). Method B, reaction time $4 \mathrm{~h}$. The crude product was purified by crystallization in methanol. 4f: yellow solid $\mathrm{Mp}=103-104{ }^{\circ} \mathrm{C}$ (lit. 105-107 $\left.{ }^{\circ} \mathrm{C}\right)$, Yield: $2.4 \mathrm{~g}, 60 \% . R_{f}=0.45, \mathrm{SiO}_{2},(\mathrm{Cy} / \mathrm{EtOAc}$ $8 / 2) ;{ }^{1} \mathrm{H}$ NMR data are in agreement with those previously reported. ${ }^{13} \mathrm{C}$ NMR (DMSO-d6, $\left.100 \mathrm{MHz}\right): \delta=31.14\left(\mathrm{C}_{14}\right.$ and $\left.\mathrm{C}_{14}\right) ; 34.07\left(\mathrm{C}_{13}\right.$ and $\left.\mathrm{C}_{13}\right)$; $57.33\left(\mathrm{C}_{1}\right) ; 121.57\left(\mathrm{C}_{9}\right.$ and/or $\left.\mathrm{C}_{11}\right) ; 123.55\left(\mathrm{C}_{11}\right.$ and/or $\left.\mathrm{C}_{9}\right) ; 124.98\left(\mathrm{C}_{4}, \mathrm{C}_{4}, \mathrm{C}_{6}, \mathrm{C}_{6}\right) ; 128.65$ $\left(\mathrm{C}_{3}, \mathrm{C}_{3}, \mathrm{C}_{7}, \mathrm{C}_{7}\right) ; 136.77\left(\mathrm{C}_{10}\right) ; 140.13\left(\mathrm{C}_{2}\right.$ and $\left.\mathrm{C}_{2}\right) ; 148.40$ $\left(\mathrm{C}_{5}\right.$ and $\left.\mathrm{C}_{5}\right) ; 149.05\left(\mathrm{C}_{12}\right) ; 162.65\left(\mathrm{C}_{8}\right)$. HPLC purity: $95 \%$ $\left(\lambda=258 \mathrm{~nm}, R_{t}=2.76 \mathrm{~min}\right) . \mathrm{GC} / \mathrm{MS}:$ method $180 ; R_{t}=8.23$ $\min m / z: 357[\mathrm{M}]^{+}(100), 342\left[\mathrm{M}-\mathrm{CH}_{3}\right]^{+}(25), 300\left[\mathrm{M}^{+}\right.$

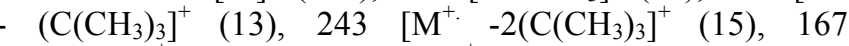
$[\mathrm{PheCHPy}]^{+}(38), 57\left[\left(\mathrm{C}\left(\mathrm{CH}_{3}\right)_{3}\right]^{+}(25)\right.$, IR $(\mathrm{ATR}): v 3050$, $3025\left(v_{\mathrm{Csp} 2-\mathrm{H}}\right), 2959,2901,2866\left(v_{\mathrm{Csp} 3-\mathrm{H}}\right), 1586,1568,1511$, 151, $1467\left(v_{\mathrm{C}=\mathrm{C}}\right), 821\left(\delta_{\mathrm{Csp} 2-\mathrm{H} \text { p-disubst }}\right)$. HRMS: calcd. for $\mathrm{C}_{26} \mathrm{H}_{31} \mathrm{~N}[\mathrm{M}+\mathrm{H}]^{+}$(358.25293); found (358.25323).

\subsubsection{Preparation of 2-[bis(4-aminophenyl)-methyl] pyri- dine $4 \mathrm{~g}$ [47]}

To a solution of 2-pyridinecarboxaldehyde 2 (4.27 g, 40 mmol, 1 equiv) in aniline (7.84 g, $84.2 \mathrm{mmol}, 2$ equiv) was added dropwise concentrated hydrochloric acid at RT. The reaction mixture was stirred at $150{ }^{\circ} \mathrm{C}$ for $24 \mathrm{~h}$ and the reaction progress monitored by TLC (Cy/EtOAc 1/1). The reaction mixture was cooled then neutralized with a concentrated ammonia solution ( $\mathrm{pH}$ 7-8) and extracted with dichloromethane three times. The combined organic phases were washed with water then with brine, dried over $\mathrm{Na}_{2} \mathrm{SO}_{4}$, filtered and concentrated. FCC on silica gel (Cy/EtOAc 8/2) afforded the $\mathbf{4 g}$ as a white solid, $\mathrm{Mp}=171-172{ }^{\circ} \mathrm{C}$, Yield $6.94 \mathrm{~g}, 63 \% . R_{f}=0.57, \mathrm{SiO}_{2}$, (EtOAc); HPLC purity: $99 \%$, (Heptane/ AcOEt 3/7, $\lambda=260 \mathrm{~nm}, R_{t}=9.72 \mathrm{~min}$ ). GC/MS: method 200; $R_{t}=6.99 \mathrm{~min} \mathrm{~m} / z: 275[\mathrm{M}]^{+}(59), 197[\mathrm{M}-\mathrm{Py}]^{+}$ (100), IR (ATR): 3459, 3426, 3354, $3328\left(v_{\mathrm{NH} 2}\right), v$ 3052, $3027\left(v_{\text {Csp2-H }}\right), 2998,2919,\left(v_{\text {Csp3-H }}\right), 1620,1587,1514,1462$ $\left(v_{\mathrm{C}=\mathrm{C}}\right), 815\left(\delta_{\mathrm{Csp} 2-\mathrm{H} \text { p-disubst }}\right)$. LRMS (ESI, CV $\left.=25\right): 276.31$ for $\mathrm{C}_{18} \mathrm{H}_{17} \mathrm{~N}_{3}[\mathrm{M}+\mathrm{H}]^{+}$(100); HRMS: calcd. for $\mathrm{C}_{18} \mathrm{H}_{17} \mathrm{~N}_{3}$ $[\mathrm{M}+\mathrm{H}]^{+}(276.14952)$; found $(276.14997)$. NMR data are in agreement with those previously reported (see SI).

\subsubsection{Preparation of 2-[bis(4- acetylaminophenyl)- methyl/pyridine 6}

To a solution of 2-[bis(4-aminophenyl)-methyl]pyridine $4 \mathrm{~g}$ (2 g, $7.26 \mathrm{mmol}, 1$ equiv) in dichloromethane $(73 \mathrm{~mL})$ was added acetic anhydride (1.56 g, $15.25 \mathrm{mmol}, 2.1$ equiv). The reaction mixture was stirred at $\mathrm{RT}$ and the reaction progress may be monitored by TLC (8:2 Cy/EtOAc). After 30 minutes the reaction mixture was concentrated then neutralized with saturated $\mathrm{Na}_{2} \mathrm{CO}_{3}$ solution $(\mathrm{pH}$ 7-8) and washed with water. The organic phase was concentrated and ovendried. 6 white solid $\mathrm{Mp}=229-230{ }^{\circ} \mathrm{C}$, Yield $2.6 \mathrm{~g}, 99 \% . R_{f}=$ $0.24, \mathrm{SiO}_{2}$, (EtOAc); ${ }^{1} \mathrm{H} \mathrm{NMR}\left(\mathrm{CD}_{3} \mathrm{OD}, 400 \mathrm{MHz}\right) \delta=2.09$ $\left(\mathrm{s}, 6 \mathrm{H}, \mathrm{CH}_{3}\right) ; 5.60\left(\mathrm{~s}, 1 \mathrm{H}, \mathrm{H}_{1}\right) ; 7.02\left(\mathrm{~d}, 4 \mathrm{H}, \mathrm{J}_{\text {ortho }}=8.5 \mathrm{~Hz}\right.$, $\left.\mathrm{H}_{3}, \mathrm{H}_{3}, \mathrm{H}_{7}, \mathrm{H}_{7}\right) ; 7.11\left(\mathrm{dm}, 1 \mathrm{H}, \mathrm{J}_{9-10}=7.9 \mathrm{~Hz}, \mathrm{H}_{9}\right) ; 7.24$ $\left(\mathrm{ddd}, 1 \mathrm{H}, \mathrm{J}_{11-9}=1.0 \mathrm{~Hz}, \mathrm{~J}_{11-10}=7.5 \mathrm{~Hz}, \mathrm{~J}_{11-12}=5.0 \mathrm{~Hz}, \mathrm{H}_{11}\right)$; $7.46\left(\mathrm{~d}, 4 \mathrm{H}, \mathrm{J}_{\text {ortho }}=8.6 \mathrm{~Hz}, \mathrm{H}_{4}, \mathrm{H}_{4}, \mathrm{H}_{6}, \mathrm{H}_{6}\right) ; 7.71(\mathrm{ddd}, 1 \mathrm{H}$, $\left.\mathrm{J}_{10-12}=1.7 \mathrm{~Hz}, \mathrm{~J}_{10-11}=\mathrm{J}_{10-9}=7.7 \mathrm{~Hz}, \mathrm{H}_{10}\right) ; 8.45(\mathrm{ddd}, 1 \mathrm{H}$, $\left.\mathrm{J}_{12-11}=5.0 \mathrm{~Hz}, \mathrm{~J}_{12-10}=1.7 \mathrm{~Hz}, \mathrm{~J}_{12-9}=0.8 \mathrm{~Hz}, \mathrm{H}_{12}\right) ;{ }^{13} \mathrm{C} \mathrm{NMR}$ 
$\left(\mathrm{CD}_{3} \mathrm{OD}, 100 \mathrm{MHz}\right): \delta=23.8\left(\mathrm{CH}_{3}\right) ; 58.6\left(\mathrm{C}_{1}\right) ; 120.9\left(\mathrm{C}_{4}\right.$, $\left.\mathrm{C}_{4}, \mathrm{C}_{6}, \mathrm{C}_{6}\right) ; 121.8\left(\mathrm{C}_{11}\right) ; 125.1\left(\mathrm{C}_{9}\right) ; 130.3\left(\mathrm{C}_{3}, \mathrm{C}_{3}, \mathrm{C}_{7}\right.$, $\left.\mathrm{C}_{7}\right) ; 137.9\left(\mathrm{C}_{2}, \mathrm{C}_{2}\right) ; 138.4\left(\mathrm{C}_{10}\right) ; 138.9\left(\mathrm{C}_{5}, \mathrm{C}_{5},\right) ; 149.3$ $\left(\mathrm{C}_{12}\right)$; $163.7\left(\mathrm{C}_{8}\right) ; 171.1\left(\mathrm{C}_{14}\right)$. HPLC purity: $95 \%$, (heptane/AcOEt $\left.3 / 70 /, \lambda=260 \mathrm{~nm}, R_{t}=9.72 \mathrm{~min}\right)$. IR (ATR): 3298, 3261 $\left(v_{\mathrm{NH}}\right), 3184,3122,3050\left(v_{\mathrm{Csp} 2 \mathrm{H}}\right), 2926,2869$ $\left(v_{\mathrm{Csp} 3-\mathrm{H}}\right), 1671\left(v_{\mathrm{c}=\mathrm{o}}\right), 1603,1536,1505,1440\left(\mathrm{v}_{\mathrm{C}=\mathrm{C}}\right), 818$ $\left(\delta_{\text {Csp2-H p-disubst }}\right)$. LRMS (ESI, CV $\left.=25\right): 360.34[\mathrm{M}+\mathrm{H}]^{+}$ (100), $382.28[\mathrm{M}+\mathrm{Na}]^{+}$(36); HRMS: calcd. for $\mathrm{C}_{22} \mathrm{H}_{21} \mathrm{~N}_{3} \mathrm{O}_{2}$ $[\mathrm{M}+\mathrm{H}]^{+}(360.17065)$; found (360.17082).

\subsubsection{General Procedure for the Preparation of Diarylme- thylpyridines $\mathrm{N}$-oxides 7 and $8 \mathrm{~b}$}

To a solution of the diarylmethylpyridines 4 or 5 (1 equiv) in acetic acid $(1.1 \mathrm{~mL} / \mathrm{mmol})$ was added $35 \% \mathrm{H}_{2} \mathrm{O}_{2}(5$ equiv). The reaction mixture was stirred at $80{ }^{\circ} \mathrm{C}$ and the reaction progress monitored by TLC (Cy/EtOAc). After reaction completion, the reaction mixture was cooled to RT and excess $\mathrm{H}_{2} \mathrm{O}_{2}$ was reduced using a saturated solution of sodium thiosulfate pentahydrate. The reaction mixture was neutralized with saturated $\mathrm{NaHCO}_{3} / \mathrm{Na}_{2} \mathrm{CO}_{3}$ solution $(1 / 1)$ and extracted with dichloromethane three times. The combined organic phases were dried over anhydrous $\mathrm{Na}_{2} \mathrm{SO}_{4}$, filtered and concentrated. If required, the crude residue was purified by FCC on silica gel to afford the corresponding diarylmethylpyridines $N$-oxides 7 or $\mathbf{8 b}$ : Yields: 7a, $80 \%$; 7b, 93\%; 7c, 70\%; 7d, 40\%, 7e, 69\%; 7f, 98\%; 7g, 12\%; 7h, $13 \% ; 8 \mathbf{b}, 84 \%$.

\subsubsection{2-(bis(4-fluorophenyl)methyl)pyridine 1-oxide 7 a}

From $4 \mathbf{a}(293 \mathrm{mg}, 1 \mathrm{mmol})$. Reaction time $3 \mathrm{~h}$. Yield 7a: $247 \mathrm{mg}, 80 \%$, yellow solid, $\mathrm{Mp}=76-77^{\circ} \mathrm{C}, R_{f}=0.22, \mathrm{SiO}_{2}$, (EtOAc). ${ }^{1} \mathrm{H}$ NMR $\left(\mathrm{CDCl}_{3}, 400 \mathrm{MHz}\right) \delta=6.3\left(\mathrm{~s}, 1 \mathrm{H}, \mathrm{H}_{1}\right)$; 6.90-6.96 (m, 1H, H $)$; 6.97-7.08 (m, 8H, H4, $\mathrm{H}_{4}, \mathrm{H}_{6}, \mathrm{H}_{6}$, $\left.\mathrm{H}_{3}, \mathrm{H}_{3}, \mathrm{H}_{7}, \mathrm{H}_{7}\right)$; 7.18-7.22 (m, 2H, $\left.\mathrm{H}_{10}, \mathrm{H}_{11}\right) ; 8.25-8.31(\mathrm{~m}$, $\left.1 \mathrm{H}, \mathrm{H}_{12}\right) ;{ }^{13} \mathrm{C} \mathrm{NMR}\left(\mathrm{CDCl}_{3}, 100 \mathrm{MHz}\right): \delta=49.1\left(\mathrm{C}_{1}\right) ; 115.8$ $\left(\mathrm{d}, 4 \mathrm{C}, J_{\mathrm{C}-\mathrm{F}}=21.4 \mathrm{~Hz}, \mathrm{C}_{4}, \mathrm{C}_{4}, \mathrm{C}_{6}, \mathrm{C}_{6}\right) ; 124.2\left(\mathrm{C}_{11}\right) ; 125.5$ $\left(\mathrm{C}_{9}\right) ; 126.7\left(\mathrm{C}_{10}\right) ; 130.7\left(\mathrm{~d}, 4 \mathrm{C}, J_{\mathrm{C}-\mathrm{F}}=7.9 \mathrm{~Hz}, \mathrm{C}_{3}, \mathrm{C}_{3}, \mathrm{C}_{7}\right.$, $\left.\mathrm{C}_{7}\right) ; 135.8\left(\mathrm{~d}, 2 \mathrm{C}, J_{\mathrm{C}-\mathrm{F}}=3.1 \mathrm{~Hz}, \mathrm{C}_{2}, \mathrm{C}_{2}\right)$ ); $139.9\left(\mathrm{C}_{12}\right) ; 154.1$ $\left(\mathrm{C}_{8}\right) ; 160.5\left(\mathrm{~d}, 2 \mathrm{C}, J_{\mathrm{C}-\mathrm{F}}=244.9 \mathrm{~Hz}, \mathrm{C}_{5}, \mathrm{C}_{5}\right.$ ). HPLC purity: $98 \%$, (heptane/AcOEt $\left.2 / 8, \lambda=274 \mathrm{~nm}, R_{t}=23.9 \mathrm{~min}\right)$. IR (ATR): 3069, 3038, ( $\left.v_{\mathrm{Csp} 2-\mathrm{H}}\right), 2922\left(v_{\mathrm{Csp} 3-\mathrm{H}}\right), 1603,1506$, $1485\left(\mathrm{v}_{\mathrm{C}=\mathrm{C}}\right), 1219\left(\mathrm{v}_{\mathrm{N}}^{+}{ }_{-\mathrm{O}}^{-}\right), 1157\left(\mathrm{v}_{\mathrm{C}-\mathrm{F}}\right), 822\left(\delta_{\mathrm{Csp} 2-\mathrm{H}}\right.$ p-disubst $)$. LRMS (ESI, $\mathrm{CV}=55): 298.28[\mathrm{M}+\mathrm{H}]^{+}$(89), 320.22 $[\mathrm{M}+\mathrm{Na}]^{+}$(100); HRMS: calcd. for $\mathrm{C}_{18} \mathrm{H}_{13} \mathrm{~F}_{2} \mathrm{NO}[\mathrm{M}+\mathrm{Na}]^{+}$ (320.08574); found (320.08569).

\subsubsection{2-(bis(4-chlorophenyl)methyl)pyridine 1-oxide $7 b$}

From 4b (316 mg, $1 \mathrm{mmol})$ Reaction time $3 \mathrm{~h}$. Yield $\mathbf{7 b}$ : $308 \mathrm{mg}, 93 \%$, yellow solid, $\mathrm{Mp}=140-141^{\circ} \mathrm{C}, R_{f}=0.27$, $\mathrm{SiO}_{2}$, (EtOAc); ${ }^{1} \mathrm{H} \mathrm{NMR}\left(\mathrm{CDCl}_{3}, 400 \mathrm{MHz}\right) \delta=6.24(\mathrm{~s}, 1 \mathrm{H}$, $\left.\mathrm{H}_{1}\right)$; 6.92-6.94 (m, $\left.1 \mathrm{H}, \mathrm{H}_{9}\right) ; 7.03\left(\mathrm{~d}, 4 \mathrm{H}, J_{\text {ortho }}=8.5 \mathrm{~Hz}, \mathrm{H}_{3}\right.$, $\mathrm{H}_{3}, \mathrm{H}_{7}, \mathrm{H}_{7}$ ); 7.18-7.23 (m, 2H, $\mathrm{H}_{10}$ and $\left.\mathrm{H}_{11}\right) ; 7.30(\mathrm{~d}, 4 \mathrm{H}$, $J_{\text {ortho }}=8.4 \mathrm{~Hz}, \mathrm{H}_{4}, \mathrm{H}_{4}, \mathrm{H}_{6}, \mathrm{H}_{6}$ ) $; 8.26-8.28\left(\mathrm{~m}, 1 \mathrm{H}, \mathrm{H}_{12}\right) ;{ }^{13} \mathrm{C}$ NMR $\left(\mathrm{CDCl}_{3}, 100 \mathrm{MHz},\right): \delta=49.5\left(\mathrm{C}_{1}\right) ; 124.4\left(\mathrm{C}_{11}\right) ; 125.5$ $\left(\mathrm{C}_{9}\right) ; 126.8\left(\mathrm{C}_{10}\right) ; 129.1\left(\mathrm{C}_{3}, \mathrm{C}_{3}, \mathrm{C}_{7}, \mathrm{C}_{7}\right) ; 130.6\left(\mathrm{C}_{4}, \mathrm{C}_{4}, \mathrm{C}_{6}\right.$, $\left.\mathrm{C}_{6}\right) ; 133.4\left(\mathrm{C}_{5}\right.$ and $\left.\mathrm{C}_{5}\right) ; 138.3\left(\mathrm{C}_{2}, \mathrm{C}_{2}\right) ; 140.0\left(\mathrm{C}_{12}\right) ; 153.5$ $\left(\mathrm{C}_{8}\right)$. IR (ATR): 3113, 3090, $3049\left(\mathrm{v}_{\mathrm{Csp} 2-\mathrm{H}}\right), 2920\left(\mathrm{v}_{\mathrm{Csp} 3-\mathrm{H}}\right)$, $1487,1430\left(v_{\mathrm{C}=\mathrm{C}}\right), 1238\left(v_{\mathrm{N}}^{+}{ }_{-0}^{-}\right), 810\left(\delta_{\mathrm{Csp} 2-\mathrm{H} \text { p-disubst }}\right)$. LRMS $\left(\mathrm{ESI}, \mathrm{CV}=4\right.$ 5): $330.18[\mathrm{M}+\mathrm{H}]^{+}(63), 352.13[\mathrm{M}-\mathrm{H}+\mathrm{Na}]^{+}$ (100); HRMS: calcd. for $\mathrm{C}_{18} \mathrm{H}_{13} \mathrm{Cl}_{2} \mathrm{NO} \quad[\mathrm{M}+\mathrm{Na}]^{+}$ (352.02664); found (352.02674).

\subsubsection{2-(bis(4-bromophenyl)methyl)pyridine 1-oxide 7c}

From 4c (402 mg, $1 \mathrm{mmol})$. Reaction time $4 \mathrm{~h}$. Yield 7c: $292 \mathrm{mg}, 70 \%$, yellow solid, $\mathrm{Mp}=152-153^{\circ} \mathrm{C}, R_{f}=0.28$, $\mathrm{SiO}_{2},\left(\right.$ EtOAc); ${ }^{1} \mathrm{H} \mathrm{NMR}\left(\mathrm{CDCl}_{3}, 400 \mathrm{MHz}\right) \delta=6.2(\mathrm{~s}, 1 \mathrm{H}$, $\left.\mathrm{H}_{1}\right) ; 6.95\left(\mathrm{~d}, 4 \mathrm{H}, J_{\text {ortho }}=8.4 \mathrm{~Hz}, \mathrm{H}_{3}, \mathrm{H}_{3}, \mathrm{H}_{7}, \mathrm{H}_{7}\right.$ ); 6.92-6.99 $\left(\mathrm{m}, 1 \mathrm{H}, \mathrm{H}_{9}\right)$; 7.24-7.32 (m, 2H, $\left.\mathrm{H}_{11}, \mathrm{H}_{10}\right) ; 7.44\left(\mathrm{~d}, 4 \mathrm{H}, J_{\text {ortho }}=\right.$ $\left.8.4 \mathrm{~Hz}, \mathrm{H}_{4}, \mathrm{H}_{4}, \mathrm{H}_{6}, \mathrm{H}_{6}\right)$; 8.36-8.38 (m, $\left.1 \mathrm{H}, \mathrm{H}_{12}\right) ;{ }^{13} \mathrm{C} \mathrm{NMR}$ $\left(\mathrm{CDCl}_{3}, 100 \mathrm{MHz}\right): \delta=49.6\left(\mathrm{C}_{1}\right) ; 121.6\left(\mathrm{C}_{5}, \mathrm{C}_{5},\right) ; 124.5$ $\left(\mathrm{C}_{11}\right) ; 126.9\left(\mathrm{C}_{9}\right) ; 127.4\left(\mathrm{C}_{10}\right) ; 131.0\left(\mathrm{C}_{3}, \mathrm{C}_{3}, \mathrm{C}_{7}, \mathrm{C}_{7}\right) ; 132.1$ $\left(\mathrm{C}_{4}, \mathrm{C}_{4}, \mathrm{C}_{6}, \mathrm{C}_{6}\right) ; 138.5\left(\mathrm{C}_{2}, \mathrm{C}_{2}\right) ; 140.3\left(\mathrm{C}_{12}\right) ; 175.2\left(\mathrm{C}_{8}\right)$. HPLC purity: 97\%, (heptane/AcOEt $2 / 8,1 \mathrm{~mL} \mathrm{~min}^{-1}, \lambda=$ $\left.276 \mathrm{~nm}, R_{t}=14.32 \mathrm{~min}\right)$. IR (ATR): 3108, 3081, 3039, 3014 $\left(v_{\mathrm{Csp} 2-\mathrm{H}}\right), 2965,2853\left(v_{\mathrm{Csp} 3-\mathrm{H}}\right), 1483,1426\left(v_{\mathrm{C}=\mathrm{C}}\right), 1237,\left(v_{\mathrm{N}}^{+}{ }_{-}\right.$ $\left.\mathrm{o}^{-}\right), 870\left(\delta_{\mathrm{Csp} 2-\mathrm{H}}\right.$ p-disubst $)$. LRMS (ESI, CV $\left.=45\right): 420.05$ $[\mathrm{M}+\mathrm{H}]^{+}(42), 442.06[\mathrm{M}+\mathrm{Na}]^{+}(100)$; HRMS: calcd. for $\mathrm{C}_{18} \mathrm{H}_{13} \mathrm{Br}_{2} \mathrm{NO}[\mathrm{M}+\mathrm{Na}]^{+}$(441.92356); found (441.92366)

\subsubsection{2-(bis(4-iodophenyl)methyl)pyridine 1-oxide 7d}

From $4 d(500 \mathrm{mg}, 1 \mathrm{mmol})$ in acetic acid $(1.4 \mathrm{~mL})$ was added $\mathrm{H}_{2} \mathrm{O}_{2} 35 \%(0.47 \mathrm{~mL}, 5 \mathrm{mmol}, 5$ equiv $)$. Reaction time $24 \mathrm{~h}$. The crude was purified by FCC on silica gel (Cy/EtOAc 3/7). Yield 7d: $207 \mathrm{mg}, 40 \%$, white solid, $\mathrm{Mp}=$ 207-208 ${ }^{\circ} \mathrm{C}, R_{f}=0.38, \mathrm{SiO}_{2},(\mathrm{EtOAc}) ;{ }^{1} \mathrm{H} \mathrm{NMR}\left(\mathrm{CDCl}_{3}, 400\right.$ $\mathrm{MHz}) \delta=6.17\left(\mathrm{~s}, 1 \mathrm{H}, \mathrm{H}_{1}\right) ; 6.83\left(\mathrm{~d}, 4 \mathrm{H}, J_{\text {ortho }}=8.4 \mathrm{~Hz}, \mathrm{H}_{3}\right.$, $\mathrm{H}_{3}, \mathrm{H}_{7}, \mathrm{H}_{7}$ ) ; $6.92\left(\mathrm{dd}, 1 \mathrm{H}, J_{9-10}=7.0 \mathrm{~Hz}, J_{9-11}=2.8 \mathrm{~Hz}, \mathrm{H}_{9}\right.$ ); 7.15-7.24 (m, $2 \mathrm{H}, \mathrm{H}_{10}$ and $\left.\mathrm{H}_{11}\right) ; 7.64\left(\mathrm{~d}, 4 \mathrm{H}, J_{\text {ortho }}=8.4 \mathrm{~Hz}\right.$, $\left.\mathrm{H}_{4}, \mathrm{H}_{4}, \mathrm{H}_{6}, \mathrm{H}_{6}\right) ; 8.27\left(\mathrm{dd}, 1 \mathrm{H}, J_{12-11}=5.5 \mathrm{~Hz}, J_{12-10}=2.0\right.$ $\left.\mathrm{Hz}, \mathrm{H}_{12}\right) ;{ }^{13} \mathrm{C} \mathrm{NMR}\left(\mathrm{CDCl}_{3}, 100 \mathrm{MHz}\right): \delta=49.5\left(\mathrm{C}_{1}\right) ; 92.8$ $\left(\mathrm{C}_{5}, \mathrm{C}_{5}\right) ; 124.1$ and $125.3\left(\mathrm{C}_{10}\right.$ and $\left.\mathrm{C}_{11}\right) ; 126.5\left(\mathrm{C}_{9}\right) ; 130.9$ $\left(\mathrm{C}_{3}, \mathrm{C}_{3}, \mathrm{C}_{7}, \mathrm{C}_{7}\right)$; $137.7\left(\mathrm{C}_{4}, \mathrm{C}_{4}, \mathrm{C}_{6}, \mathrm{C}_{6}\right) ; 139.0\left(\mathrm{C}_{2}, \mathrm{C}_{2},\right)$; $139.6\left(\mathrm{C}_{12}\right) ; 152.9\left(\mathrm{C}_{8}\right)$. HPLC purity: $99 \%$ ( heptane/AcOEt

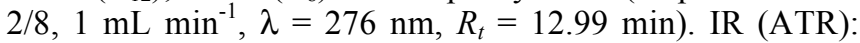
3100, 3074, 3035, 3009 ( $\left.v_{\mathrm{Csp} 2-\mathrm{H}}\right), 2958,2825\left(v_{\mathrm{Csp} 3-\mathrm{H}}\right), 1481$, $1423\left(v_{\mathrm{C}=\mathrm{C}}\right), 1239,\left(v_{\mathrm{N}}^{+}{ }_{-\mathrm{O}}^{-}\right), 841\left(\delta_{\mathrm{Csp} 2-\mathrm{H} \text {-disubst }}\right)$. LRMS (ESI, $\mathrm{CV}=45): 514.04(100),[\mathrm{M}+\mathrm{H}]^{+},=535.99(22)\left[\mathrm{M}+\mathrm{Na}^{+}\right]$, HRMS: calcd. for $\mathrm{C}_{18} \mathrm{H}_{13} \mathrm{I}_{2} \mathrm{NO}[\mathrm{M}+\mathrm{Na}]^{+}$(535.89787); found (535.89769).

\subsubsection{2-(bis(4-methylphenyl)methyl)pyridine 1-oxide 7e}

From 4e (500 mg, $1.83 \mathrm{mmol})$. Reaction time $3.5 \mathrm{~h}$. The crude was purified by $\mathrm{FCC}$ on silica gel $\left(\mathrm{CH}_{2} \mathrm{Cl}_{2} / \mathrm{MeOH}\right.$ 96/4) Yield 7e: $367 \mathrm{mg}, 69 \%$, white solid, $\mathrm{Mp}=142-143{ }^{\circ} \mathrm{C}$, $R_{f}=0.33, \mathrm{SiO}_{2},\left(\mathrm{CH}_{2} \mathrm{Cl}_{2} / \mathrm{MeOH}, 96 / 4\right) ;{ }^{1} \mathrm{H}$ NMR (DMSOd6, $400 \mathrm{MHz}): \delta=2.30\left(\mathrm{~s}, 6 \mathrm{H}, \mathrm{CH}_{3}\right) ; 6.12\left(\mathrm{~s}, 1 \mathrm{H}, \mathrm{H}_{1}\right) ; 6.97$ $\left(\mathrm{d}, 4 \mathrm{H}, J_{\text {ortho }}=8.1 \mathrm{~Hz}, \mathrm{H}_{3}, \mathrm{H}_{3}, \mathrm{H}_{7}, \mathrm{H}_{7}\right) ; 7.15\left(\mathrm{~d}, 4 \mathrm{H}, J_{\text {ortho }}=8\right.$ $\mathrm{Hz}, \mathrm{H}_{4}, \mathrm{H}_{4}, \mathrm{H}_{6}, \mathrm{H}_{6}$ ); 7.17-7.25 (m, 1H, H $\left.\mathrm{H}_{11}\right) ; 7.26$ (ddd, $1 \mathrm{H}$, $\left.J_{9-12}=1.2 \mathrm{~Hz}, J_{9-11}=7.8 \mathrm{~Hz}, J_{9-10}=8.7 \mathrm{~Hz}, \mathrm{H}_{9}\right) ; 7.35(\mathrm{ddd}$, $\left.1 \mathrm{H}, J_{10-12}=1.6 \mathrm{~Hz}, J_{10-11}=7.6 \mathrm{~Hz}, J_{10-9}=8.7 \mathrm{~Hz}, \mathrm{H}_{10}\right) ; 8.30$ $\left(\mathrm{dd}, 1 \mathrm{H}, J_{12-11}=6.5 \mathrm{~Hz}, J_{12-10}=1.6 \mathrm{~Hz}, \mathrm{H}_{12}\right) ;{ }^{13} \mathrm{C} \mathrm{NMR}$ (DMSO-d6, $100 \mathrm{MHz}): \delta=20.58\left(\mathrm{CH}_{3}\right) ; 48.93\left(\mathrm{C}_{1}\right) ; 124.45$ $\left(\mathrm{C}_{9}\right.$ and/or $\left.\mathrm{C}_{11}\right) ; 126.52\left(\mathrm{C}_{11}\right.$ and/or $\left.\mathrm{C}_{9}\right) ; 128.80\left(\mathrm{C}_{3}, \mathrm{C}_{3}, \mathrm{C}_{7}\right.$, $\left.\mathrm{C}_{7}\right) ; 129.09\left(\mathrm{C}_{4}, \mathrm{C}_{4}, \mathrm{C}_{6}, \mathrm{C}_{6}\right) ; 135.79\left(\mathrm{C}_{5}\right.$ and $\left.\mathrm{C}_{5}\right) ; 137.73$ $\left(\mathrm{C}_{2}\right.$ and $\left.\mathrm{C}_{2}\right) ; 139.12\left(\mathrm{C}_{10}\right) ; 139.24\left(\mathrm{C}_{12}\right) ; 152.91\left(\mathrm{C}_{8}\right)$. HPLC purity: $95 \%$, (heptane/AcOEt 2/8, $1 \mathrm{~mL} \cdot \mathrm{min}^{-1}, \lambda=276 \mathrm{~nm}$, $\left.R_{t}=15.61 \mathrm{~min}\right)$. IR (ATR): 3090 and $3082\left(\mathrm{v}_{\mathrm{Csp} 2-\mathrm{H}}\right), 2918$ and $2870\left(v_{\mathrm{Csp} 3-\mathrm{H}}\right), 1512,1482,1427\left(\mathrm{v}_{\mathrm{C}=\mathrm{C}}\right), 1239,\left(\mathrm{v}_{\mathrm{N}}^{+}{ }_{-}^{-}\right)$, $841\left(\delta_{\text {Csp2-H p-disubst }}\right)$. HRMS: calcd. for $\mathrm{C}_{20} \mathrm{H}_{19} \mathrm{NO}[\mathrm{M}+\mathrm{Na}]^{+}$ (312.13589); found (312.13583).

\subsubsection{2-(bis(4-tert-butylphenyl)methyl)pyridine 1-oxide $7 f$}

From 4 f $(500 \mathrm{mg}, 1.40 \mathrm{mmol}$ Reaction time $3.5 \mathrm{~h}$. Yield 7f: $0.51 \mathrm{~g}, 98 \%$, yellow solid, $\mathrm{Mp}=167-168^{\circ} \mathrm{C}, R_{f}=0.34$, 
$\mathrm{SiO}_{2}$, $\left(\mathrm{CH}_{2} \mathrm{Cl}_{2} / \mathrm{MeOH}, 95 / 5\right) ;{ }^{1} \mathrm{H}$ NMR (DMSO-d6, 400 MHz) $\delta=1.29\left(\mathrm{~s}, 18 \mathrm{H}, \mathrm{H}_{14}\right) ; 6.12\left(\mathrm{~s}, 1 \mathrm{H}, \mathrm{H}_{1}\right) ; 7.02(\mathrm{~d}, 4 \mathrm{H}$, $\left.\mathrm{J}_{\text {ortho }}=7.8 \mathrm{~Hz}, \mathrm{H}_{3}, \mathrm{H}_{3}, \mathrm{H}_{7}, \mathrm{H}_{7}\right)$; 7.27-7.32 (m, $3 \mathrm{H}, \mathrm{H}_{9}, \mathrm{H}_{10}$, $\left.\mathrm{H}_{11}\right) ; 7.36\left(\mathrm{~d}, 4 \mathrm{H}, \mathrm{J}_{\text {ortho }}=7.9 \mathrm{~Hz}, \mathrm{H}_{4}, \mathrm{H}_{4}, \mathrm{H}_{6}, \mathrm{H}_{6}\right) ; 8.29(\mathrm{~d}$, $\left.1 \mathrm{H}, \mathrm{J}_{12-11}=6.3 \mathrm{~Hz}, \mathrm{H}_{12}\right) ;{ }^{13} \mathrm{C}$ NMR (DMSO-d6, $100 \mathrm{MHz}$ ): $\delta=31.11\left(\mathrm{C}_{14}\right.$ and $\left.\mathrm{C}_{14}\right) ; 34.13\left(\mathrm{C}_{13}\right.$ and $\left.\mathrm{C}_{13}\right) ; 48.76\left(\mathrm{C}_{1}\right)$; $124.45\left(\mathrm{C}_{9}\right.$ and/or $\left.\mathrm{C}_{11}\right) ; 124.55\left(\mathrm{C}_{11}\right.$ and/or $\left.\mathrm{C}_{9}\right) ; 125.29\left(\mathrm{C}_{4}\right.$, $\left.\mathrm{C}_{4}, \mathrm{C}_{6}, \mathrm{C}_{6}\right) ; 126.47\left(\mathrm{C}_{10}\right) ; 128.59\left(\mathrm{C}_{3}, \mathrm{C}_{3}, \mathrm{C}_{7}, \mathrm{C}_{7}\right) ; 137.68$ $\left(\mathrm{C}_{2}\right.$ and $\left.\mathrm{C}_{2}\right) ; 139.13\left(\mathrm{C}_{12}\right) ; 148.91\left(\mathrm{C}_{5}\right.$ and $\left.\mathrm{C}_{5}\right) ; 152.82$ $\left(\mathrm{C}_{8}\right)$ HPLC purity: 90\%, Hypersil Si, Heptane/ AcOEt 20:80,

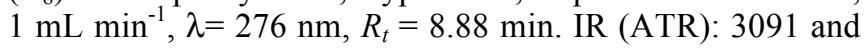
$3050\left(v_{\text {Csp2-H }}\right), 2959$ and $2866\left(v_{\text {Csp3-H }}\right), 1509,1482,1459$, $1423\left(v_{\mathrm{C}=\mathrm{C}}\right), 1238,\left(\mathrm{v}_{\mathrm{N}}^{+}{ }^{+}{ }^{-}\right), 845\left(\delta_{\mathrm{Csp} 2-\mathrm{H} \text {-disubstitution }}\right)$. LRMS (ESI, CV=45): 374.34 (100), $[\mathrm{M}+\mathrm{H}]^{+}, 396.28$ (90) $\left[\mathrm{M}+\mathrm{Na}^{+}\right]$, HRMS: calcd. for $\mathrm{C}_{26} \mathrm{H}_{31} \mathrm{NO} \quad[\mathrm{M}+\mathrm{Na}]^{+}$ (396.22979); found (396.22990).

\subsubsection{2-(bis(4-acetoxyphenyl)methyl)pyridine 1-oxide $7 \mathrm{~g}$ and 2-((4-acetoxyphenyl)(4-hydroxyphenyl)methyl)pyri- dine 1-oxide $7 \mathrm{~h}$}

From BSL 1 ( $1 \mathrm{~g}, 2.76 \mathrm{mmol})$. Reaction time $1.5 \mathrm{~h}$. The crude product was purified by FCC on silica gel $\left(\mathrm{CH}_{2} \mathrm{Cl}_{2} / \mathrm{MeOH} 96 / 4\right)$ Yield7g: $129 \mathrm{mg}, 12 \%$, orange solid (and 2-((4-acetoxyphenyl)(4-hydroxyphenyl)methyl)pyridine 1-oxide $7 \mathrm{~h}, 108 \mathrm{mg}, 12 \%$, orange solid) $\mathrm{Mp}=204-205{ }^{\circ} \mathrm{C}$, $R_{f}=0.30, \mathrm{SiO}_{2},\left(\mathrm{CH}_{2} \mathrm{Cl}_{2} / \mathrm{MeOH}, 96 / 4\right) ;{ }^{1} \mathrm{H}$ NMR DMSO-d6, $400 \mathrm{MHz},) \delta=2.28\left(\mathrm{~s}, 6 \mathrm{H}, \mathrm{CH}_{3}\right) ; 6.22\left(\mathrm{~s}, 1 \mathrm{H}, \mathrm{H}_{1}\right) ; 7.01-7.08$ (m, $\left.1 \mathrm{H}, \mathrm{H}_{9}\right) ; 7.10-7.16\left(\mathrm{~m}, 8 \mathrm{H}, \mathrm{H}_{3}, \mathrm{H}_{3}, \mathrm{H}_{4}, \mathrm{H}_{4}, \mathrm{H}_{6}, \mathrm{H}_{6}, \mathrm{H}_{7}\right.$, $\mathrm{H}_{7}$ ); 7.32-7.41 (m, $2 \mathrm{H}, \mathrm{H}_{10}$ and $\left.\mathrm{H}_{11}\right) ; 8.31-8.33(\mathrm{dm}, 1 \mathrm{H}$, $\left.J_{12-11}=5.2 \mathrm{~Hz}, \mathrm{H}_{12}\right) ;{ }^{13} \mathrm{C}$ NMR DMSO-d6, $\left.100 \mathrm{MHz},\right): \delta=$ $20.84\left(\mathrm{CH}_{3}\right) ; 48.63\left(\mathrm{C}_{1}\right) ; 121.97\left(\mathrm{C}_{4}, \mathrm{C}_{4}, \mathrm{C}_{6}, \mathrm{C}_{6}\right) ; 124.78$ $\left(\mathrm{C}_{10}\right.$ and $\left.\mathrm{C}_{11}\right) ; 126.52\left(\mathrm{C}_{9}\right) ; 129.88\left(\mathrm{C}_{3}, \mathrm{C}_{3}, \mathrm{C}_{7}, \mathrm{C}_{7}\right) ; 137.85$ $\left(\mathrm{C}_{2}\right.$ and $\left.\mathrm{C}_{2}\right) ; 139.25\left(\mathrm{C}_{12}\right) ; 149.27\left(\mathrm{C}_{5}\right.$ and $\left.\mathrm{C}_{5}\right) ; 152.28\left(\mathrm{C}_{8}\right)$; $169.18\left(\mathrm{C}_{13}\right.$ and $\left.\mathrm{C}_{13}\right)$. HPLC purity: $99 \%$, (Hypersil ODS C18, methanol $\left./ \mathrm{H}_{2} \mathrm{O} 9 / 1, \lambda=263 \mathrm{~nm}, R_{t}=2.22 \mathrm{~min}\right)$. IR (ATR): 3100, $3115\left(v_{\text {Csp2-H }}\right), 2962,2915,2848\left(v_{\text {Csp3-H }}\right)$, $1751\left(v_{\mathrm{c}=\mathrm{o}}\right), 1507,1484,1426\left(v_{\mathrm{C}=\mathrm{C}}\right), 1202\left(\mathrm{v}_{\mathrm{N}}{ }^{+}-\mathrm{O}^{-}\right), 853$

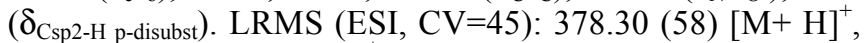
$400.27(100),[\mathrm{M}+\mathrm{Na}]^{+}$. HRMS: calcd. for $\mathrm{C}_{22} \mathrm{H}_{19} \mathrm{NO}_{5}$ $[\mathrm{M}+\mathrm{Na}]^{+} \quad$ (400.11554); found (400.11571). 2-((4acetoxyphenyl)(4-hydroxyphenyl)methyl)pyridine 1oxide $7 \mathbf{h}: \mathrm{Mp}=208-209^{\circ} \mathrm{C}, R_{f}=0.23, \mathrm{SiO}_{2},\left(\mathrm{CH}_{2} \mathrm{Cl}_{2} / \mathrm{MeOH}\right.$, 96:4); ${ }^{1} \mathrm{H}$ NMR (DMSO-d6, $400 \mathrm{MHz}$,) $\delta=2.29$ (s, 3H, $\left.\mathrm{CH}_{3}\right) ; 6.09\left(\mathrm{~s}, 1 \mathrm{H}, \mathrm{H}_{1}\right) ; 6.77\left(\mathrm{~d}, 2 \mathrm{H}, J_{16-17}=J_{19-20}=8.4 \mathrm{~Hz}\right.$, $\mathrm{H}_{17}$ and $\left.\mathrm{H}_{19}\right) ; 6.91\left(\mathrm{~d}, 2 \mathrm{H}, J_{16-17}=J_{19-20}=8.4 \mathrm{~Hz}, \mathrm{H}_{16}\right.$ and $\left.\mathrm{H}_{20}\right) ; 7.00\left(\mathrm{dd}, 1 \mathrm{H}, \mathrm{J}_{9-11}=2.1 \mathrm{~Hz}, J_{9-10}=7.7 \mathrm{~Hz}, \mathrm{H}_{9}\right) ; 7.10(\mathrm{~m}$, $\left.4 \mathrm{H}, \mathrm{H}_{3}, \mathrm{H}_{4}, \mathrm{H}_{6}, \mathrm{H}_{7}\right) ; 7.30-7.40\left(\mathrm{~m}, 2 \mathrm{H}, \mathrm{H}_{10}\right.$ and $\left.\mathrm{H}_{11}\right) ; 8.29$ (d, $\left.1 \mathrm{H}, J_{12-11}=6.2 \mathrm{~Hz}, \mathrm{H}_{12}\right) ; 9.46\left(\mathrm{~s}_{\mathrm{br}}, 1 \mathrm{H}, \mathrm{OH}\right) ;{ }^{13} \mathrm{C} \mathrm{NMR}$ (DMSO-d6, $100 \mathrm{MHz}$ ): $\delta=20.85\left(\mathrm{CH}_{3}\right) ; 48.37\left(\mathrm{C}_{1}\right) ; 115.41$ $\left(\mathrm{C}_{17}\right.$ and $\left.\mathrm{C}_{19}\right) ; 121.79\left(\mathrm{C}_{4}\right.$ and $\left.\mathrm{C}_{6}\right) ; 124.52\left(\mathrm{C}_{10}\right.$ and/or $\left.\mathrm{C}_{11}\right)$; $124.65\left(\mathrm{C}_{11}\right.$ and/or $\left.\mathrm{C}_{10}\right) ; 126.51\left(\mathrm{C}_{9}\right) ; 129.70$ and $130.08\left(\mathrm{C}_{3}\right.$, $\left.\mathrm{C}_{7}, \mathrm{C}_{16}, \mathrm{C}_{20}\right) ; 130.53\left(\mathrm{C}_{2}\right.$ and/or $\left.\mathrm{C}_{15}\right) ; 138.54\left(\mathrm{C}_{15}\right.$ and/or $\mathrm{C}_{2}$ ); $139.16\left(\mathrm{C}_{12}\right) ; 149.05\left(\mathrm{C}_{5}\right) ; 153.0\left(\mathrm{C}_{8}\right) ; 156.27\left(\mathrm{C}_{18}\right) ; 169.22$ $\left(\mathrm{C}_{13}\right)$. IR (ATR): $3065\left(\mathrm{v}_{\mathrm{Csp} 2-\mathrm{H}}\right), 2922,2797\left(\mathrm{v}_{\mathrm{Csp} 3-\mathrm{H}}\right), 1751$ $\left(v_{\mathrm{c}=\mathrm{O}}\right), 1614,1515\left(\mathrm{v}_{\mathrm{C}=\mathrm{C}}\right), 1223\left(\mathrm{v}_{\mathrm{N}}^{+} \mathrm{O}^{-}\right), 844\left(\delta_{\mathrm{Csp2} 2 \mathrm{H} \mathrm{p} \text {-disubst }}\right)$. LRMS (ESI, CV = 45): $336.31(50)[\mathrm{M}+\mathrm{H}]^{+}, 358.25(100)$, $[\mathrm{M}+\mathrm{Na}]^{+}$. HRMS: calcd. for $\mathrm{C}_{20} \mathrm{H}_{17} \mathrm{NO}_{4} \quad[\mathrm{M}+\mathrm{Na}]^{+}$ (358.10498); found (358.10503).

\subsubsection{2-((2-chlorophenyl)(4-chlorophenyl)methyl)pyridine 1-oxide $8 b$}

From $5 \mathbf{b}(157 \mathrm{mg}, 0.5 \mathrm{mmol})$. Reaction time $3 \mathrm{~h}$. Yield 8b: $138 \mathrm{mg}, 84 \%$, white solid, $\mathrm{Mp}=202-203{ }^{\circ} \mathrm{C}, R_{f}=0.32$,
$\mathrm{SiO}_{2},\left(\right.$ EtOAc) ${ }^{1} \mathrm{H}$ NMR $\left(\mathrm{CDCl}_{3}, 400 \mathrm{MHz}\right) \delta=6.52(\mathrm{~s}, 1 \mathrm{H}$, $\left.\mathrm{H}_{1}\right) ; 6.82\left(\mathrm{dd}, 1 \mathrm{H}, \mathrm{J}_{3-4}=7.6 \mathrm{~Hz}, \mathrm{~J}_{3-5}=1.8 \mathrm{~Hz}, \mathrm{H}_{3}\right) ; 6.85(\mathrm{dd}$, $\left.1 \mathrm{H}, J_{9-10}=7.6 \mathrm{~Hz}, J_{9-11}=2.4 \mathrm{~Hz}, \mathrm{H}_{9}\right) ; 7.03\left(\mathrm{~d}, 2 \mathrm{H}, J_{\mathrm{ortho}}=8.3\right.$ $\left.\mathrm{Hz}, \mathrm{H}_{3}, \mathrm{H}_{7}\right) ; 7.15-7.25\left(\mathrm{~m}, 4 \mathrm{H}, \mathrm{H}_{11}, \mathrm{H}_{10}, \mathrm{H}_{5}, \mathrm{H}_{4}\right) ; 7.30(\mathrm{~d}$, $\left.2 \mathrm{H}, J_{\text {ortho }}=8.3 \mathrm{~Hz}, \mathrm{H}_{4}, \mathrm{H}_{6}\right) ; 7.42\left(\mathrm{dd}, 1 \mathrm{H}, J_{6-5}=7.8 \mathrm{~Hz}, J_{6-4}\right.$ $\left.=1.5 \mathrm{~Hz}, \mathrm{H}_{6}\right) ; 8.28\left(\mathrm{dd}, 1 \mathrm{H}, J_{12-11}=6.4 \mathrm{~Hz}, J_{12-10}=1.4 \mathrm{~Hz}\right.$, $\left.\mathrm{H}_{12}\right) ;{ }^{13} \mathrm{C}$ NMR $\left(\mathrm{CDCl}_{3}, 100 \mathrm{MHz}\right): \delta=47.8\left(\mathrm{C}_{1}\right) ; 124.4$ $\left(\mathrm{C}_{11}\right) ; 125.4\left(\mathrm{C}_{10}\right) ; 126.5\left(\mathrm{C}_{9}\right) ; 127.0$ and $128.8\left(\mathrm{C}_{5}\right.$ and $\left.\mathrm{C}_{4}\right)$; $129.2\left(\mathrm{C}_{3}, \mathrm{C}_{7}\right) ; 129.8\left(\mathrm{C}_{3}\right) ; 130.4\left(\mathrm{C}_{6}\right) ; 130.8\left(\mathrm{C}_{4}, \mathrm{C}_{6}\right)$; $133.4\left(\mathrm{C}_{5}\right) ; 135.1\left(\mathrm{C}_{7}\right) ; 137.2\left(\mathrm{C}_{2}\right) ; 137.9\left(\mathrm{C}_{2}\right) ; 140.0\left(\mathrm{C}_{12}\right)$; $152.7\left(\mathrm{C}_{8}\right)$. HPLC purity: 91\%, (heptane/ AcOEt $2 / 8,1 \mathrm{~mL}$ $\left.\min ^{-1}, \lambda=276 \mathrm{~nm}, R_{t}=11.39 \mathrm{~min}\right)$. IR (ATR): 3080, 3048, $3008\left(v_{\mathrm{Csp} 2-\mathrm{H}}\right), 2917,2850\left(v_{\mathrm{Csp} 3-\mathrm{H}}\right), 1482,1465,1429\left(v_{\mathrm{C}=\mathrm{C}}\right)$, $1238,\left(v_{\mathrm{N}-{ }^{-}}\right), 823\left(\delta_{\text {Csp2-H p-disubst }}\right), 755\left(\delta_{\text {Csp2-H o-disubst }}\right)$. LRMS $(\mathrm{ESI}, \mathrm{CV}=45): 330.18[\mathrm{M}+\mathrm{H}]^{+}(96), 352.13[\mathrm{M}-\mathrm{H}+\mathrm{Na}]^{+}$ (100); HRMS: calcd. for $\mathrm{C}_{18} \mathrm{H}_{13} \mathrm{Cl}_{2} \mathrm{NO} \quad[\mathrm{M}+\mathrm{Na}]^{+}$ (352.02664); found (352.02674).

\subsubsection{2-(bis(4-acetylaminophenyl)methyl)pyridine 1-oxide 9 and 2-((4-acetylaminophenyl)(4-nitrophenyl)methyl) pyridine 1-oxide 10}

From 6 (1.8 g, 5 mmol, 1 equiv). Reaction time 4.5 h. After workup the crude product was purified by FCC on silica gel $\left(\mathrm{CH}_{2} \mathrm{Cl}_{2} / \mathrm{MeOH}\right.$ 95/5). Yield 9: $839 \mathrm{mg}, 45 \%$ (and 10: $376 \mathrm{mg}, 21 \%)$. 2-(bis(4-acetylaminophenyl) methyl) pyridine 1-oxide 9: yellowish solid, $\mathrm{Mp}=261-262^{\circ} \mathrm{C}, R_{f}=0.26$, $\mathrm{SiO}_{2},\left(\mathrm{CH}_{2} \mathrm{Cl}_{2} / \mathrm{MeOH}, 9 / 1\right) ;{ }^{1} \mathrm{H}$ NMR $\left(\mathrm{CD}_{3} \mathrm{OD}, 400 \mathrm{MHz}\right) \delta$ $=2.10\left(\mathrm{~s}, 6 \mathrm{H}, \mathrm{CH}_{3}\right) ; 6.17\left(\mathrm{~s}, 1 \mathrm{H}, \mathrm{H}_{1}\right) ; 6.98\left(\mathrm{~d}, 4 \mathrm{H}, J_{\text {ortho }}=8.6\right.$ $\left.\mathrm{Hz}, \mathrm{H}_{3}, \mathrm{H}_{3}, \mathrm{H}_{7}, \mathrm{H}_{7}{ }^{\prime}\right) ; 7.07\left(\mathrm{dd}, 1 \mathrm{H}, J_{9-10}=7.8 \mathrm{~Hz}, J_{9-11}=2.0\right.$ $\left.\mathrm{Hz}, \mathrm{H}_{9}\right) ; 7.31-7.43\left(\mathrm{~m}, 2 \mathrm{H}, \mathrm{H}_{11}, \mathrm{H}_{10}\right) ; 7.49\left(\mathrm{~d}, 4 \mathrm{H}, J_{\text {ortho }}=8.6\right.$ $\left.\mathrm{Hz}, \mathrm{H}_{4}, \mathrm{H}_{4}, \mathrm{H}_{6}, \mathrm{H}_{6}\right) ; 8.26\left(\mathrm{~d}, 1 \mathrm{H}, J_{12-11}=6.0 \mathrm{~Hz}, \mathrm{H}_{12}\right) ;{ }^{13} \mathrm{C}$ NMR $\left(\mathrm{CD}_{3} \mathrm{OD}, 100 \mathrm{MHz}\right): \delta=23.8\left(\mathrm{CH}_{3}\right) ; 50.2\left(\mathrm{C}_{1}\right) ; 121.0$ $\left(\mathrm{C}_{3}, \mathrm{C}_{3}, \mathrm{C}_{7}, \mathrm{C}_{7}\right) ; 125.4\left(\mathrm{C}_{11}\right) ; 128.2\left(\mathrm{C}_{9}\right) ; 129.1\left(\mathrm{C}_{10}\right) ; 130.1$ $\left(\mathrm{C}_{4}, \mathrm{C}_{4}, \mathrm{C}_{6}, \mathrm{C}_{6}\right) ; 136.1\left(\mathrm{C}_{5}, \mathrm{C}_{5}\right) ; 138.3\left(\mathrm{C}_{2}, \mathrm{C}_{2}\right) ; 140.3$ $\left(\mathrm{C}_{12}\right) ; 155.2\left(\mathrm{C}_{8}\right) ; 171.0\left(\mathrm{C}_{13}\right)$. HPLC purity: 100\% (Hypersil ODS C18, methanol $\left./ \mathrm{H}_{2} \mathrm{O} 9 / 1, \lambda=255 \mathrm{~nm}, R_{t}=2.05 \mathrm{~min}\right)$. IR (ATR): $3329\left(v_{\mathrm{NH}}\right) 3117\left(v_{\text {Csp2-H }}\right), 2921,2859\left(v_{\text {Csp3-H }}\right)$, $1676\left(v_{\mathrm{c}=0}\right), 1609,1550,1510,1479\left(v_{\mathrm{C}=\mathrm{C}}\right), 1226,\left(v_{\mathrm{N}}{ }^{+}{ }^{-}\right)$,

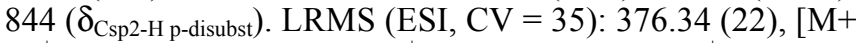
$\mathrm{H}]^{+}, 398.30$ (100), [M+ Na $]^{+}, 414.33$ (25), [M+K $\left.{ }^{+}\right]$, HRMS: calcd. for $\mathrm{C}_{22} \mathrm{H}_{21} \mathrm{~N}_{3} \mathrm{O}_{3} \quad[\mathrm{M}+\mathrm{Na}]^{+} \quad(398.14751)$; found (398.14770). 2-[(4-acetylaminophenyl)(4-nitrophenyl) methyl]pyridine 1-oxide 10 lemon yellow solid, $\mathrm{Mp}=261$ $262{ }^{\circ} \mathrm{C}, R_{f}=0.49, \mathrm{SiO}_{2},\left(\mathrm{CH}_{2} \mathrm{Cl}_{2} / \mathrm{MeOH}, 9 / 1\right) ;{ }^{1} \mathrm{H} \mathrm{NMR}$ (DMSO-d6, $400 \mathrm{MHz}) \delta=2.00\left(\mathrm{~s}, 3 \mathrm{H}, \mathrm{CH}_{3}\right) ; 6.15(\mathrm{~s}, 1 \mathrm{H}$, $\left.\mathrm{H}_{1}\right) ; 6.95\left(\mathrm{dd}, 1 \mathrm{H}, J_{9-10}=7.9 \mathrm{~Hz}, J_{9-11}=2.0 \mathrm{~Hz}, \mathrm{H}_{9}\right) ; 7.02(\mathrm{~d}$, $\left.2 \mathrm{H}, J_{\text {ortho }}=8.6 \mathrm{~Hz}, \mathrm{H}_{3}, \mathrm{H}_{7}\right) ; 7.26-7.32\left(\mathrm{~m}, 3 \mathrm{H}, \mathrm{H}_{3}, \mathrm{H}_{7}, \mathrm{H}_{10}\right)$; 7.36 (ddd, $1 \mathrm{H}, \mathrm{J}_{11-10}=7.7 \mathrm{~Hz}, \mathrm{~J}_{11-12}=6.5 \mathrm{~Hz}, \mathrm{~J}_{11-9}=2.0 \mathrm{~Hz}$, $\left.\mathrm{H}_{11}\right) ; 7.52\left(\mathrm{~d}, 2 \mathrm{H}, J_{\text {ortho }}=8.6 \mathrm{~Hz}, \mathrm{H}_{4}, \mathrm{H}_{6}\right) ; 8.15\left(\mathrm{~d}, 2 \mathrm{H}, J_{\text {ortho }}\right.$ $\left.=8.8 \mathrm{~Hz}, \mathrm{H}_{4}, \mathrm{H}_{6}\right) ; 8.26\left(\mathrm{dd}, 1 \mathrm{H}, J_{12-11}=6.4 \mathrm{~Hz}, J_{12-10}=0.8\right.$ $\left.\mathrm{Hz}, \mathrm{H}_{12}\right) ;{ }^{13} \mathrm{C}$ NMR (DMSO-d6, $100 \mathrm{MHz}$ ): $\delta=24.0\left(\mathrm{CH}_{3}\right)$; $49.4\left(\mathrm{C}_{1}\right) ; 119.4\left(\mathrm{C}_{4}, \mathrm{C}_{6}\right) ; 123.7\left(\mathrm{C}_{4}, \mathrm{C}_{6}\right) ; 124.9\left(\mathrm{C}_{10}\right) ; 125.1$ $\left(\mathrm{C}_{11}\right) ; 126.7\left(\mathrm{C}_{9}\right) ; 129.6\left(\mathrm{C}_{3}, \mathrm{C}_{7}\right) ; 129.8\left(\mathrm{C}_{3}, \mathrm{C}_{7}\right) ; 133.7$ $\left(\mathrm{C}_{5}\right) ; 138.5\left(\mathrm{C}_{2}\right) ; 139.3\left(\mathrm{C}_{12}\right) ; 146.2\left(\mathrm{C}_{5}\right) ; 148.8\left(\mathrm{C}_{2}\right) ; 151.6$ $\left(\mathrm{C}_{8}\right) ; 168.3\left(\mathrm{C}_{13}\right)$. HPLC purity: 100\% (Hypersil ODS C18, methanol $\left./ \mathrm{H}_{2} \mathrm{O} 9 / 1, \lambda=257 \mathrm{~nm}, R_{t}=9.72 \mathrm{~min}\right)$. IR (ATR): $3255\left(v_{\mathrm{NH}}\right) 3183,3052\left(v_{\mathrm{Csp} 2-\mathrm{H}}\right), 2929\left(v_{\mathrm{Csp} 3-\mathrm{H}}\right), 1670\left(v_{\mathrm{c}=\mathrm{o}}\right)$, $1599,1511,1486\left(v_{\mathrm{C}=\mathrm{C}}\right), 1228\left(\mathrm{v}_{\mathrm{N}}^{+}{ }^{+}{ }^{-}\right)$. LRMS (ESI, CV $=$ 35): 364.31 (19), $[\mathrm{M}+\mathrm{H}]^{+}, 386.25$ (100), $[\mathrm{M}+\mathrm{Na}]^{+}, 387.26$ (23), [M+H+Na], HRMS: calcd. for $\mathrm{C}_{20} \mathrm{H}_{17} \mathrm{~N}_{3} \mathrm{O}_{4}[\mathrm{M}+\mathrm{Na}]^{+}$ (386.11144); found (386.11113). 


\subsubsection{2-(bis(4-aminophenyl)methyl)pyridine 1-oxide 11}

2-[Bis(4-acetylaminophenyl)methyl]pyridine 1-oxide 9 (204 mg, $0.54 \mathrm{mmol}, 1$ equiv) was dissolved in of $1.2 \mathrm{M} \mathrm{HCl}$ $(10 \mathrm{~mL})$ and the reaction mixture stirred for $2 \mathrm{~h}$ under reflux. The reaction progress may be monitored by TLC $\left(\mathrm{CH}_{2} \mathrm{Cl}_{2} / \mathrm{MeOH}, 9 / 1\right)$. After reaction completion, the reaction mixture was cooled to RT, then neutralized with saturated $\mathrm{Na}_{2} \mathrm{CO}_{3}$ solution ( $\mathrm{pH}$ 7-8) and extracted with dichloromethane three times. The combined organic phases were dried over anhydrous $\mathrm{Mg}_{2} \mathrm{SO}_{4}$, filtered and concentrated. The crude residue was purified by FCC on silica gel $\left(\mathrm{CH}_{2} \mathrm{Cl}_{2} / \mathrm{MeOH} 100 / 0\right.$ to $\left.95 / 5\right)$. Yield 11: $134 \mathrm{mg}, 85 \%$, yellow solid, $\mathrm{Mp}=229-230 \quad{ }^{\circ} \mathrm{C}, \quad R_{f}=0.45, \quad \mathrm{SiO}_{2}$, $\left(\mathrm{CH}_{2} \mathrm{Cl}_{2} \mathrm{MeOH}, 9 / 1\right) ;{ }^{1} \mathrm{H}$ NMR $\left(\mathrm{CDCl}_{3}, 400 \mathrm{MHz},\right) \delta=5.71$ (s, $\left.1 \mathrm{H}, \mathrm{H}_{1}\right) ; 6.34\left(\mathrm{~d}, 4 \mathrm{H}, J_{\text {ortho }}=8.5 \mathrm{~Hz}, \mathrm{H}_{4}, \mathrm{H}_{4}, \mathrm{H}_{6}, \mathrm{H}_{6}\right.$ ); $6.50\left(\mathrm{~d}, 4 \mathrm{H}, J_{\text {ortho }}=8.3 \mathrm{~Hz}, \mathrm{H}_{3}, \mathrm{H}_{3}, \mathrm{H}_{7}, \mathrm{H}_{7}\right) ; 6.78(\mathrm{dd}, 1 \mathrm{H}$, $\left.J_{9-10}=7.7 \mathrm{~Hz}, J_{9-11}=2.0 \mathrm{~Hz}, \mathrm{H}_{9}\right) ; 6.98\left(\mathrm{ddd}, 1 \mathrm{H}, J_{11-10}=7.7\right.$ $\left.\mathrm{Hz}, J_{11-12}=6.5 \mathrm{~Hz}, J_{11-9}=2.0 \mathrm{~Hz}, \mathrm{H}_{11}\right) ; 7.05$ (ddd, $1 \mathrm{H}, J_{10-9}=$ $\left.J_{10-11}=7.7 \mathrm{~Hz}, J_{10-12}=1.2 \mathrm{~Hz}, \mathrm{H}_{10}\right) ; 7.91\left(\mathrm{dd}, 1 \mathrm{H}, J_{12-11}=6.4\right.$ $\left.\mathrm{Hz}, J_{12-10}=0.8 \mathrm{~Hz}, \mathrm{H}_{12}\right) ;{ }^{13} \mathrm{C} \mathrm{NMR}\left(\mathrm{CDCl}_{3}, 100 \mathrm{MHz},\right): \delta=$ $48.5\left(\mathrm{C}_{1}\right) ; 115.3\left(\mathrm{C}_{4}, \mathrm{C}_{4}, \mathrm{C}_{6}, \mathrm{C}_{6}\right) ; 123.7\left(\mathrm{C}_{11}\right) ; 127.0\left(\mathrm{C}_{9}\right)$; $127.8\left(\mathrm{C}_{10}\right) ; 129.4\left(\mathrm{C}_{3}, \mathrm{C}_{3^{\prime}}, \mathrm{C}_{7}, \mathrm{C}_{7}\right) ; 129.7\left(\mathrm{C}_{2}, \mathrm{C}_{2}\right) ; 139.0$ $\left(\mathrm{C}_{12}\right) ; 145.2\left(\mathrm{C}_{5}, \mathrm{C}_{5}\right) ; 155.3\left(\mathrm{C}_{8}\right)$. HPLC purity: $99 \%$ (Hypersil ODS C18, methanol $/ \mathrm{H}_{2} \mathrm{O} 9 / 1, \lambda=260 \mathrm{~nm}, R_{t}=$ $9.72 \mathrm{~min})$. IR (ATR): 3415, $3322\left(v_{\mathrm{NH} 2}\right) 3191,3021\left(v_{\mathrm{Csp} 2-\mathrm{H}}\right)$, 2923, $2852\left(v_{\text {Csp3-H }}\right), 1642,1612,1513,1483\left(v_{\mathrm{C}=\mathrm{C}}\right), 1282$ $\left(v_{\mathrm{C}-\mathrm{N}}\right), 1219\left(v_{\mathrm{N}-\mathrm{O}^{-}}^{+}\right), 844\left(\delta_{\text {Csp2-H p-disubst }}\right)$. LRMS (ESI, CV $=$ 25): 292.29 (100), $[\mathrm{M}+\mathrm{H}]^{+}, 314.30(8),[\mathrm{M}+\mathrm{Na}]^{+}$. HRMS: calcd. for $\mathrm{C}_{18} \mathrm{H}_{17} \mathrm{~N}_{3} \mathrm{O} \quad[\mathrm{M}+\mathrm{H}]^{+} \quad(292.14444) ;$ found (292.14505).

\subsection{In Vivo Biological Evaluation}

\subsubsection{LPS-enhanced Leukocyte Migration Assay in Zebrafish}

General: All chemicals were first dissolved in dimethylsulfoxide (DMSO) 99.5\% (GC; Sigma-Aldrich), and then diluted in $0.3 \mathrm{X}$ Danieau's medium $(17.4 \mathrm{mM} \mathrm{NaCl}, 0.21$ $\mathrm{mM} \mathrm{KCl}, 0.18 \mathrm{mM} \mathrm{Ca}\left(\mathrm{NO}_{3}\right)_{2}, 0.12 \mathrm{mM} \mathrm{MgSO}_{4}, 1.5 \mathrm{mM}$ Hepes pH 7.6 and 0.03 M methylene blue). The final concentration of DMSO did not exceed $1 \%$ in order to avoid damage to the animal [48]. During the course of the experiments, transgenic zebrafish of the line fli-1: EGFP were used. Once the fertilized eggs were collected, they were reared in $0.3 \mathrm{X}$ Danieau's medium at $28{ }^{\circ} \mathrm{C}$. At $20 \mathrm{~h}$ post fertilization, larvae were exposed to $0.2 \mathrm{mM}$ 1-phenyl-2thiourea (PTU; Sigma- Aldrich) to suppress melanization [49].

Toxicological evaluation: The Maximum Tolerated Concentration (MTC) was defined as the maximum concentration at which no death or sign of toxicity in the zebrafish larvae at doses evaluated. The MTC was initially determined by incubating 4-dpf zebrafish larvae with different concentrations of the compounds. During $24 \mathrm{~h}, 5$ larvae per well were placed in a 24-well culture plate and incubated with concentrations of $3 \mu \mathrm{M}, 10 \mu \mathrm{M}, 30 \mu \mathrm{M}$ and $100 \mu \mathrm{M}$. Larvae were then observed under a light microscope (Carl Zeiss Stemi 2000C) each hour during a period of eight hours and a final evaluation at $24 \mathrm{~h}$ post-incubation. Changes in the normal morphology and physiology of larvae were considered as signs of toxicity. All the toxicological assays were performed by duplicate using a different group of zebrafish larvae per set of experiments.

Anti-inflammatory procedure. The anti-inflammatory activity was determined by in vivo LPS-enhanced leukocyte migration assay in zebrafish. Ten 4-dpf larvae were used per experimental group and controls. The positive control (indomethacin) and negative control (DMSO 1\%) were tested per set of samples. Larvae were incubated in a total volume of $1 \mathrm{~mL}$. After an initial incubation of $1 \mathrm{~h}$, were anesthetized by immersion in $0.3 \mathrm{X}$ Danieau's medium containing 70 $\mu \mathrm{g} / \mathrm{mL}$ tricaine (ethyl 3-aminobenzoate; Sigma-Aldrich) to perform a complete tail transaction in the tail tip region. Once tails were cut, larvae were subjected to further incubation for $7 \mathrm{~h}$ at $28^{\circ} \mathrm{C}$. At this stage, the inclusion of $10 \mu \mathrm{g} / \mathrm{mL}$ of LPS from Salmonella typhosa (ATCC 10749, SigmaAldrich), ensures stimulation of the leukocyte migration to the damaged zone. When the incubation time was completed, larvae were fixed overnight in $4 \%$ formaldehyde (SigmaAldrich) at $4{ }^{\circ} \mathrm{C}$. A series of washes with Phosphate Buffer Saline Plus 1\% Tween 20 (PBST) followed the fixation process. Posteriorly, the colouring reaction tookplace using 1 $\mathrm{mL}$ of staining solution of Leucognost POX ${ }^{\circledR}$ (Merck) for 15 min. Microscopic evaluation of each larva for every group allowed a semi-quantitative analysis, where values of Relative Leukocyte Migration (RLM) were expressed for every sample [50].

\subsubsection{TPA-induced Mouse Ear Oedema in Mice}

Animals: Housing condition and experimental protocols were approved by the Institutional Ethics Committee of the University of Valencia, (Spain) in accordance with the guidelines established by the European Union on Animal Care (CEE Council 86/609). Female BALB/c mice weighing 25-30 g (Harlan Interfauna Iberica, Barcelona, Spain) were used. All animals were fed a standard diet ad libitum and housed under a $12 \mathrm{~h}$ light/dark cycle at $22{ }^{\circ} \mathrm{C}$ and $60 \% \mathrm{hu}$ midity.

Anti-inflammatory experimental test: Topical antiinflammatory activity of the compounds was studied using the method described by Young and De Young [51] and modified by Payá et al. [52]. Oedema was induced on the right ear by topical application of $2.5 \mu \mathrm{g} / \mathrm{ear}$ of $\mathrm{O}$ tetradecanoylphorbol-13-acetate (TPA) (Sigma- Aldrich) in $20 \mu \mathrm{L}$ acetone (Panreac) $(10 \mu \mathrm{L} /$ side). Vehicle-control animals received the same volume of acetone applied to the right ear. BSL and indomethacin (Sigma-Aldrich) at a dose of $0.5 \mathrm{mg} / \mathrm{ear}$ were dissolved in $20 \mu \mathrm{L}$ acetone and applied topically to right ear simultaneously with TPA. Indomethacin was used as a reference drug. Oedema was measured $4 \mathrm{~h}$ post the inflammation induction, the animals were sacrificed by cervical dislocation and right and left ear punches $(6 \mathrm{~mm}$ diameter) were taken from each mouse. The oedema was measured as an increase in ear thickness due to the TPA agent application by difference in weight between both ears. Tissues were frozen and stored at $-80{ }^{\circ} \mathrm{C}$ until use. Details of the method have been previously described [53]. Values were expressed as the increase in ear weight $(\Delta \mathrm{W})$ standard error of the mean, $\mathrm{n}=3$. Significance was determined by ANOVA followed by Dunett's test. 
Myeloperoxidase (MPO) assay in mouse ear oedema tissues: We used the method described by Young and De Young with some modifications [51]. Ear sections were placed in a tube and homogenized in $1.5 \mathrm{~mL}$ of $80 \mathrm{mM}$ sodium phosphate buffer (PBS, $\mathrm{pH} 5.4$ ) containing $0.5 \%$ hexadecyltrimethylammonium bromide. After centrifugation, PBS $(100 \mu \mathrm{L}), 22 \mathrm{mM}$ monopotassium phosphate/disodium phosphate buffer $(85 \mu \mathrm{L})$ and $0.017 \% \mathrm{H}_{2} \mathrm{O}_{2}(15 \mu \mathrm{L})$ were added to $30 \mu \mathrm{L}$ of the supernatant. MPO activity was assessed by measuring the $\mathrm{H}_{2} \mathrm{O}_{2}$-dependent oxidation of 3,3',5,5'-tetramethyl-benzidine (TMB). The reaction was stopped by addition of $30 \mu \mathrm{L} 1.46 \mathrm{M} \mathrm{NaOAc}(\mathrm{pH} 3.0)$. Enzyme activity was determined by measuring absorbance at $630 \mathrm{~nm}$. MPO activity was expressed as the amount of enzyme required to convert $1 \mu \mathrm{mol}$ of $\mathrm{H}_{2} \mathrm{O}_{2}$ to water in $1 \mathrm{~min}$ at $25^{\circ} \mathrm{C}$, expressed per gram of wet weight of tissue.

\subsubsection{Carrageenan-induced Paw Oedema in Rats}

Animals: Wistar male rats (180-200 g) from Centro para la Producción de Animales de Laboratorio (CENPALAB, La Habana, Cuba) were used. They were kept in a temperature controlled environment $\left(23{ }^{\circ} \mathrm{C}\right)$ with a $12 \mathrm{~h}$ light-dark cycle, relative humidity $40-70 \%$, with food and water ad libitum and fasted overnight $(18 \mathrm{~h})$ before the day of the experiments.

Procedure: Inflammation was produced by injecting 100 $\mu \mathrm{L}$ of a $1 \%$ carrageenan solution in normal saline into the right hind paw of the rats, according to the method of Winter et al. [54] Rats were divided into 5 groups with each consisting of six rats. The reference drug indomethacin $(10 \mathrm{mg} / \mathrm{kg})$ and BSL (10, 20 and $40 \mathrm{mg} / \mathrm{kg}$ ) were administered intraperitoneally (ip) $30 \mathrm{~min}$ before the carrageenan injection. Animals in the control group received vehicle only. The development of paw oedema was measured plethysmographically (7140 plethysmometer, Ugo Basile) up to $5 \mathrm{~h}$ after the carrageenan injection at intervals of $60 \mathrm{~min}$. The percentage of inhibition of inflammation was calculated as follow:

Percent inhibition: $\mathrm{I}=100 \times[1-(\mathrm{a}-\mathrm{x}) /(\mathrm{b}-\mathrm{y})]$ where, $\mathrm{x}$ is the mean paw volume of rats before the administration of carrageenan and test compounds or reference compound (test group), a is the mean paw volume of rats after the administration of carrageenan in the test group (drug treated), $b$ is the mean paw volume of rats after the administration of carrageenan in the control group and y is the mean paw volume of rats before the administration of carrageenan in the control group.

\subsection{In Vitro Evaluations}

\subsubsection{Cell Culture}

The RAW 264.7 cell line was obtained from the ECACC (Salisbury, UK). The cells were cultured in Dulbecco Modified Eagle's Medium (DMEM) supplemented with 10\% fetal bovine serum, penicillin $(100 \mathrm{U} / \mathrm{mL})$ and streptomycin sulfate $(100 \mu \mathrm{g} / \mathrm{mL})$ in a humidified $5 \% \mathrm{CO}_{2}$ atmosphere at 37 ${ }^{\circ} \mathrm{C}$.

BSL was dissolved in DMSO, to give a stock concentration of $10 \mathrm{mM}$ and were stored at $-20^{\circ} \mathrm{C}$. DMSO was used as a vehicle control in all experiments and the amount of DMSO $(0.1 \%, v / v)$ used was standardized in all samples.

\subsubsection{Cell Viability Assay}

The effect on cell viability of BSL was evaluated with the 3-[4,5-dimethylthiazol-2-yl]-2,5- diphenyltetrazol bromide (MTT; Sigma-Aldrich) assay. Thus, murine RAW 264.7 macrophages were exposed to BSL at 50 and $100 \mu \mathrm{M}$ in a 96-well microplate during $24 \mathrm{~h}$ at $37{ }^{\circ} \mathrm{C}$ and $5 \% \mathrm{CO}_{2}$ atmosphere after which $100 \mu \mathrm{L}$ per well of a $0.5 \mathrm{mg} / \mathrm{mL}$ solution of MTT (Sigma-Aldrich) was added, incubated at $37^{\circ} \mathrm{C}$ until blue deposits were visible and dissolved in 100 $\mu \mathrm{L}$ DMSO. Absorbance was measured at $490 \mathrm{~nm}$ with a VICTOR $^{\text {TM }} X 3$ Multilabel Plate Reader (PerkinElmer, Shelton, USA). The results were expressed in absolute absorbance readings; a decrease in absorbance indicated a reduction in cell viability. Concentrations where cell viability was inferior to $90 \%$ were considered toxic.

\subsubsection{Nitric Oxide (NO) Determination and Prostaglandin $E_{2}\left(P G E_{2}\right)$ Assay}

The RAW 264.7 cells were seeded at $5 \times 10^{5}$ cells per well in 24-well microplates. BSL (50 and $100 \mu \mathrm{M})$ was added to the culture medium $1 \mathrm{~h}$ before stimulation with 1 $\mathrm{mg} / \mathrm{mL}$ LPS. The presence of nitrites $\left(\mathrm{NO}_{2}{ }^{-}\right)$, assessed as the index of NO generation, was determined in the culture supernatant by mixing with an equal volume of Griess reagent (1\% sulfanilamide and $0.1 \%$ naphthylenediamine in $5 \%$ phosphoric acid). Absorbance at $550 \mathrm{~nm}$ was measured, and concentrations were calculated against a sodium nitrite standard curve. In the $\mathrm{PGE}_{2}$ assay, the cultured supernatant was collected $24 \mathrm{~h}$ after LPS stimulation and $\mathrm{PGE}_{2}$ production was quantified by immunoenzymatic assay kit (Cayman Chemicals, San Diego, CA, USA) according to the manufacturer's instructions.

\subsubsection{Measurement of Cytokine Production}

The RAW 264.7 cells were seeded at $5 \times 10^{5}$ cells per well in 24-well microplates, treated with BSL at concentration of $100 \mu \mathrm{M}$ and $1 \mathrm{~h}$ later stimulated with $1 \mu \mathrm{g} / \mathrm{mL}$ LPS. After $24 \mathrm{~h}$ the supernatants were collected and TNF- $\alpha$ and IL-6 production was quantified using an ELISA kit (eBioscience, San Diego, CA, USA) according to the manufacturer's instructions.

\subsubsection{Western Blot Analysis for COX-2 and Inducible Ni- tric Oxide Synthase (iNOS)}

RAW 264.7 macrophages $\left(1.5 \times 10^{6}\right.$ cells per well $)$ were co-incubated in a 6 -well culture plate $(1 \mathrm{~mL})$ with $1 \mu \mathrm{g} / \mathrm{mL}$ of LPS at $37{ }^{\circ} \mathrm{C}$ for $24 \mathrm{~h}$ in the presence of BSL or vehicle. Cellular lysates were obtained with lysis buffer (1\% Triton $\mathrm{X}-1001 \mathrm{~mL}, 1 \%$ deoxycholic acid $1 \mathrm{~g}, 20 \mathrm{mM} \mathrm{NaCl} 120$ mg, $25 \mathrm{mM}$ Tris-HCl $390 \mathrm{mg}$, and 1 tablet of complete Mini EDTA-free protease inhibitor cocktail from Roche). After centrifugation $(10,000 \times \mathrm{g}, 10 \mathrm{~min})$, the proteins present in the supernatants were quantified. Equal amounts of protein (30 $\mu \mathrm{g})$ were then loaded onto $10 \%$ sodium dodecyl sulfate polyacrylamide electrophoresis gel and were transferred onto polyvinylidene difluoride membranes at $125 \mathrm{~mA}$ for $90 \mathrm{~min}$. The membranes were then blocked in PBS-Tween 20 containing $3 \% \mathrm{w} / \mathrm{v}$ defatted milk for $1 \mathrm{~h}$ and then incubated with anti-iNOS (1/1000 dilution) and anti-COX-2 (1/2000 dilution) polyclonal antibodies (both from Cayman, Ann Arbor, MI, USA). For $\beta$-actin, used as a loading control, the mem- 
branes were incubated with anti- $\beta$-actin polyclonal antibody (1/12000) (Sigma-Aldrich). The blots were washed and incubated with peroxidase-conjugate anti-rabbit immunoglobulin $G(1 / 20000$ dilution). The immunoreactive bands were visualized with the aid of an enhanced chemiluminiscence system (GE Healthcare, Fairfield, CT, USA).

Software: Images for Western blot experiments were acquired with the image analysis system LAS-3000 mini (Fujifilm, Tokyo, Japan). Digital images were processed and band density measurements were made with the aid of a Multi Gauge V3.0 software package (Fujifilm).

Statistical analysis: Statistical analysis was performed by means of one-way analysis of variance (ANOVA) and Dunnett's t-test. The results are presented as the mean \pm S.E.M. GraphPad Prism 4.0 software (GraphPad Software Inc., San Diego, CA, USA) was used for all calculations.

\section{RESULTS AND DISCUSSION}

\subsection{Modelling Anti-Inflammatory Activity}

With the purpose of guarantying the molecular and pharmacological diversity, a dataset with a great number of molecular entities reported as anti-inflammatory and a whole group of compounds with other pharmacological reported uses were captured [24, 25]. The dataset used here was conformed and published by us in a previous study.[15] This dataset contains 1213 organic-chemicals having a great degree of structural variability. From them, 919 were chosen at random to form the training set (TS), 443 out of them being actives, and 476 inactives. The remaining sub-series, composed of 144 anti-inflammatory compounds and 150 compounds with different biological properties, were used as test sets (PS) for the validation of the models. The 587 antiinflammatory compounds, considered in this study, have different anti-inflammatory action modes and their chemical structures are shown as supporting information (Table SI1).

It is remarkable that the wide structural variability of active compounds in the training and prediction sets assures adequate extrapolation power [55] and increases the possibilities of the discovery of new leads with anti-inflammatory activity.

Two atom-based TOMOCOMD-CARDD MD families (NS $\left[{ }^{\mathrm{AP}} b_{\mathrm{k}}(\bar{x}, \bar{y})\right]$ and $\mathrm{SS}\left[{ }^{\mathrm{APs}} b_{\mathrm{k}}(\bar{x}, \bar{y})\right]$ bilinear indices) [29-34] were used. These bilinear maps use a complete scheme of atomic properties (AP), which characterizes a specific aspect of the atomic structure (and $k$ mean order, $k=$ 1-15). The weights (atomic-labels) used in this work are: atomic mass $(\mathrm{AP}=\mathrm{M})$, atomic polarizability $(\mathrm{AP}=\mathrm{P})$, atomic Mullinken electronegativity $(\mathrm{AP}=\mathrm{K})$ plus Van der Waals atomic volume $(\mathrm{AP}=\mathrm{V})$. All bilinear indices were calculated taken into account all $\mathrm{H}$-atoms in the molecule, $i . e .{ }^{\mathrm{AP}} b_{\mathrm{k}}{ }^{\mathrm{H}}$ $(\bar{x}, \bar{y})$ and ${ }^{\mathrm{APs}} b_{\mathrm{k}}{ }^{\mathrm{H}}(\bar{x}, \bar{y})$ for NS bilinear indices and their SS counterparts, respectively. Two local (L) atom-group indices for heteroatoms (group $=$ heteroatoms $(\mathrm{E}): \mathrm{E}=\mathrm{S}, \mathrm{N}, \mathrm{O}$ ), not considering $\left[{ }^{\mathrm{AP}} b_{\mathrm{kL}}\left(\bar{x}_{E}, \bar{y}_{E}\right)\right]$ and considering $\left[{ }^{\mathrm{AP}} b_{\mathrm{kL}}\right.$ $\left.\left(\bar{x}_{E}, \bar{y}_{E}\right)\right] \mathrm{H}$-atoms in the molecule, were computed too.

Using the total and group-local NS and SS bilinear indices, as independent variables, several classification functions were developed, that is 15 LDA-based QSAR models were obtained. The first seven models were developed using the NS total and local atom-based bilinear indices (Eqs. 1-7). Seven corresponding equations were achieved by using the SS MDs (Eqs. 8-14). The last one was obtained by mixing one another the sets of MDs, for instance NS and SS bilinear indices (Eq. 15). All equations of these classification models are shown in Table $\mathbf{1}$.

The model validation is other key features in good QSAR practice regarding the diagnostic of developed models [5660 ]. Predictivity can be claimed only if the model is successfully applied to the prediction of the external test series chemicals (Table 2). The robustness of all models was demonstrated with the adequate values of the rather-good classifications above 85\%. Equations $\mathbf{7}$ and $\mathbf{1 4}$ showed $86.39 \%$ and $88.78 \%$ of accuracy in the prediction series. Table 3 also depicts the principal statistical parameters for the PS. The best LDA-based QSAR is Eq. 14, with an accuracy of $88.78 \%$ vs $87.76 \%$ depicted by model 15 . Tables SI4 and Table SI5 of the Supporting Information summarize the results of the classifications of every compound (active and inactive, respectively), for all models represented by Equations 1-15.

\subsection{In Silico Repurposing: Identification of New Virtual Lead}

One of the main aims of the present report was to develop a model for predicting anti-inflammatory activity at early stages of drug discovery. LBVS of large databases using such models has emerged as an interesting strategy [12, 61, 62]

In this experiment, 30 compounds for each kind of pharmacological activity were evaluated in silico in order to see if they will exhibit virtual anti-inflammatory activity. Every individual classifier $\left(\boldsymbol{C}_{\mathrm{I}}\right)$ is fused into an ensemble classifier, $\boldsymbol{C}_{\mathbf{E}}$, through a voting system, where the individual output of $\boldsymbol{C}_{\mathrm{I}}$ are used like input of $\boldsymbol{C}_{\mathrm{E}}$, which will have a voting score for the query molecules. Finally, eight potential antiinflammatory leads (virtual hits) were selected from a large data reported in the databases Merck Index [24] and Negwer Handbook [25].

Initially, we decided to select only the compounds that show $C_{\mathrm{E}} \geq 10\left(\sim 70 \%\right.$ of $\left.C_{I} s\right)$ by "wet" evaluation. In addition, the following criteria were used for the hits' selection: a) compounds were selected as hits if the value of posterior probability of possessing anti-inflammatory activity exceeded $50 \%(\Delta \mathrm{P}>50 \%)$ by all LDA-based QSAR models (fusion approach or multi-classification system), b) If, among the compounds selected (or that will be obtained), too many similar compounds satisfied criterion 1 , then only several representative structures were selected, and c) compounds selected for experimental corroboration should be rather structurally dissimilar to well-known anti-inflammatory drugs in order to found new leads. Using this strategy, we selected Bisacodyl (BSL) as potential anti-inflammatory lead-like among eight structural-diverse compounds (Fig. 1).

BSL showed a good agreement between the in silico predictions and in vivo assays in several tests (see below). The values of $\Delta \mathrm{P} \%$ for this subset are depicted in Table 4 . BSL is 
Table 1. Discriminant models obtained with total and local non-stochastic and stochastic bilinear indices.

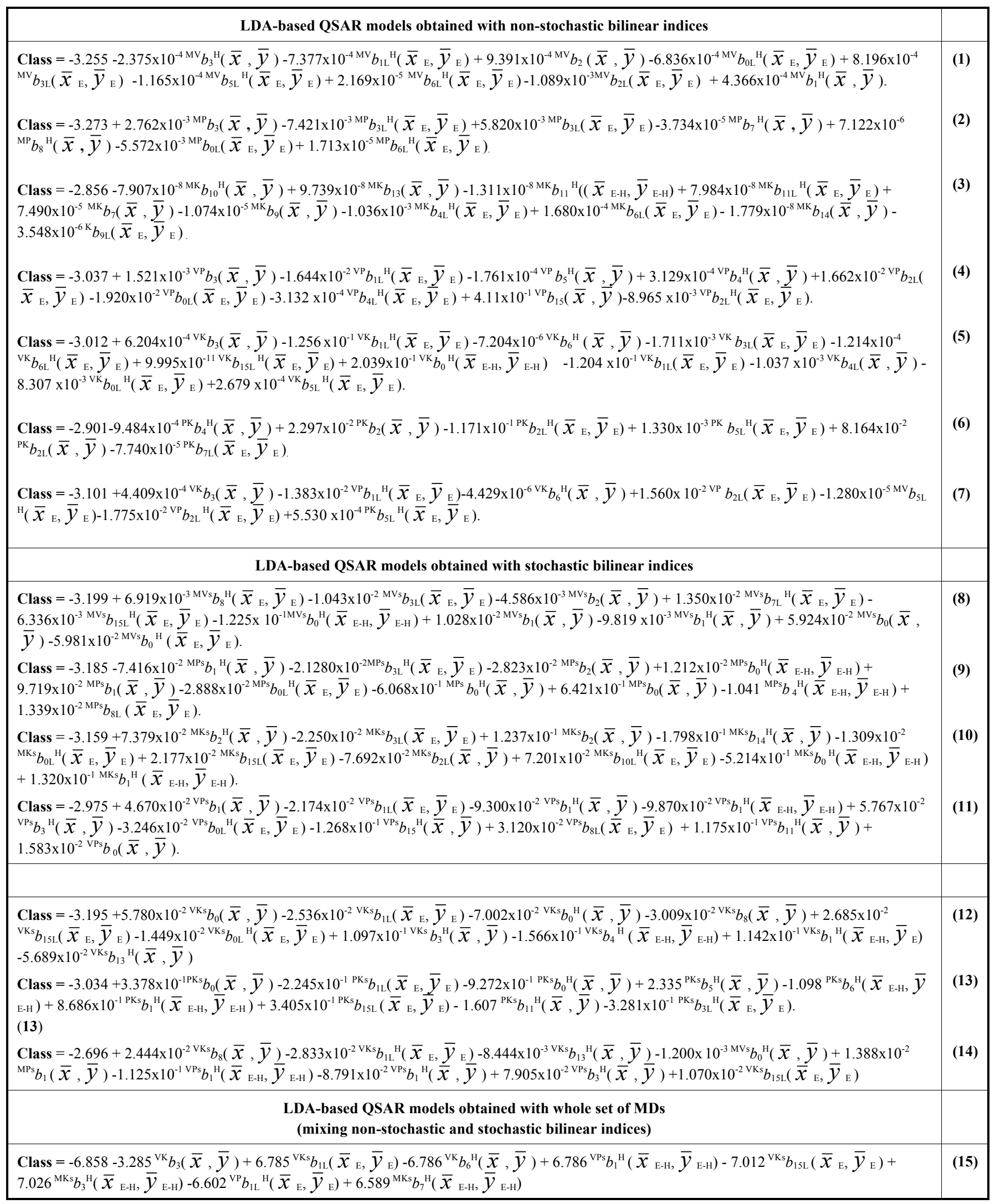


Table 2. Prediction performance and statistical parameters for LDA-based QSAR models in the training set.

\begin{tabular}{|c|c|c|c|c|c|c|c|c|}
\hline \multicolumn{9}{|c|}{ LDA-based QSAR models obtained with non-stochastic bilinear indices } \\
\hline [Eq. $2(7)]$ & 0.74 & 86.83 & 85.78 & 86.76 & 12.2 & 0.47 & 144.5 & 4.44 \\
\hline [Eq. 3(10)] & 0.73 & 86.40 & 85.33 & 86.30 & 12.6 & 0.47 & 102.2 & 4.49 \\
\hline$[$ Eq. $4(9)]$ & 0.75 & 87.49 & 87.79 & 86.00 & 11.1 & 0.46 & 118.5 & 4.69 \\
\hline [Eq. $6(6)]$ & 0.71 & 85.64 & 84.79 & 85.55 & 14.3 & 0.48 & 165.5 & 4.35 \\
\hline [Eq. 7 (7)] & 0.75 & 87.70 & 87.84 & 86.46 & 11.1 & 0.46 & 151.2 & 4.64 \\
\hline \multicolumn{9}{|c|}{ LDA-based QSAR models obtained with simple stochastic bilinear indices } \\
\hline [Eq. $8(10)]$ & 0.74 & 87.05 & 86.65 & 86.46 & 12.4 & 0.44 & 114.5 & 5.04 \\
\hline [Eq. $9(10)]$ & 0.73 & 86.72 & 86.56 & 85.78 & 12.4 & 0.47 & 101.2 & 4.45 \\
\hline [Eq.10(10)] & 0.73 & 86.62 & 86.87 & 85.10 & 12.0 & 0.47 & 92.1 & 4.50 \\
\hline [Eq. 14 (9)] & 0.74 & 87.05 & 87.16 & 85.78 & 11.8 & 0.47 & 115.5 & 4.59 \\
\hline \multicolumn{9}{|c|}{$\begin{array}{c}\text { LDA-based QSAR models obtained with whole set of MDs } \\
\text { (mixing non-stochastic and stochastic bilinear indices) }\end{array}$} \\
\hline [Eq. 15 (8)] & 0.75 & 87.70 & 87.84 & 86.46 & 11.1 & 0.44 & 143.0 & 5.02 \\
\hline
\end{tabular}

${ }^{\mathrm{a}}$ Values in parentheses indicate the quantity of variables of the models. ${ }^{\mathrm{b}}$ Matthew's correlation coefficient. ${ }^{\mathrm{c}}$ Fisher ratio. ${ }^{\mathrm{d}}$ The square Mahalanobis distance.

a well-known laxative drug indicated for the symptomatic treatment of constipation or in preparation for endoscopic examinations of the rectum and colon. It is partly absorbed by the small intestine where it undergo deacetylation and hepatic glucuronidation before returning to the digestive tract, its action is mainly engaged in the colon. Up to now, BSL 1 showed no toxicity. Some mutagenicity tests in mammalian showed no genotoxic potential [63].

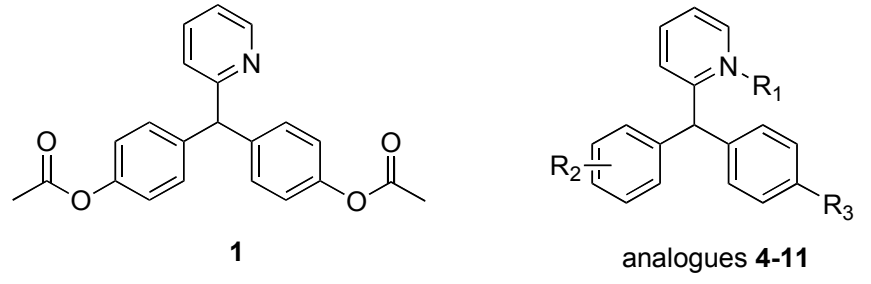

Fig. (1). Molecular structure of Bisacodyl 1 and analogues 4-11 (See Scheme 1 and Table 8) 
Table 3. Prediction performance and statistical parameters for LDA-based QSAR models in the test set.

\begin{tabular}{|c|c|c|c|c|c|}
\hline Models $^{\mathrm{a}}$ & $C^{b}$ & $\begin{array}{l}\text { Accuracy } \\
\text { “QTotal” (\%) }\end{array}$ & $\begin{array}{l}\text { Specificity } \\
\quad(\%)\end{array}$ & $\begin{array}{l}\text { Sensitivity } \\
\text { "hit rate" (\%) }\end{array}$ & $\begin{array}{c}\text { False } \\
\text { positive rate }(\%)\end{array}$ \\
\hline \multicolumn{6}{|c|}{ LDA-based QSAR models obtained with non-stochastic bilinear indices } \\
\hline [Eq. $2(7)]$ & 0.74 & 87.07 & 87.86 & 85.42 & 11.3 \\
\hline [Eq. 3(10)] & 0.72 & 86.07 & 85.62 & 84.65 & 11.5 \\
\hline [Eq. 5(11)] & 0.76 & 87.76 & 87.50 & 87.5 & 12.0 \\
\hline [Eq. $6(6)]$ & 0.71 & 85.71 & 85.42 & 85.42 & 14.0 \\
\hline [Eq. 7 ( 7)] & 0.73 & 86.39 & 86.62 & 85.42 & 12.7 \\
\hline \multicolumn{6}{|c|}{ LDA-based QSAR models obtained with stochastic bilinear indices } \\
\hline [Eq.11(10)] & 0.73 & 86.39 & 84.03 & 87.68 & 11.3 \\
\hline$[\mathrm{Eq} .12(10)]$ & 0.75 & 87.41 & 86.81 & 87.41 & 12.0 \\
\hline [Eq.13 (9) ] & 0.73 & 86.39 & 86.11 & 86.11 & 13.3 \\
\hline [Eq. 14(9)] & 0.78 & 88.78 & 88.81 & 88.19 & 10.7 \\
\hline \multicolumn{6}{|c|}{$\begin{array}{l}\text { LDA-based QSAR models obtained with whole set of MDs } \\
\text { (mixing non-stochastic and stochastic bilinear indices) }\end{array}$} \\
\hline [Eq. 15(8)] & 0.76 & 87.76 & 88.03 & 86.81 & 11.3 \\
\hline
\end{tabular}

${ }^{a}$ Values in parentheses indicate the quantity of variables of the models. ${ }^{b}$ Matthew's correlation coefficient.

Table 4. Results of ligand-based in silico screening of Bisacodyl (BSL), Carbamazepine (CBZ), Cyclocumarol (CCL), Dicumarol (DCL), Mepartricin (MPT), by using $C_{I}$.

\begin{tabular}{|c|c|c|c|c|c|c|c|c|}
\hline$\Delta \mathrm{P} \%$ & $\begin{array}{l}\text { Bisacodyl } \\
\text { (BSL 1) }\end{array}$ & $\begin{array}{c}\text { Carbamazepine } \\
\text { (CBZ) }\end{array}$ & $\begin{array}{l}\text { Cyclocumarol } \\
\text { (CCL) }\end{array}$ & $\begin{array}{l}\text { Dicumarol } \\
\text { (DCL) }\end{array}$ & $\begin{array}{l}\text { Mepartricin } \\
\text { (MPT) }\end{array}$ & $\begin{array}{l}\text { Phenolphthalein } \\
\text { (PPT) }\end{array}$ & $\begin{array}{l}\text { Protriptyline } \\
\text { hydrochloride } \\
\text { (PTH) }\end{array}$ & $\begin{array}{c}\text { Tetrandrine } \\
\text { TTD }\end{array}$ \\
\hline Eq. 1 & 98.42 & 76.65 & 96.06 & 99.19 & 99.42 & 98.74 & 80.03 & 99.85 \\
\hline Eq. 2 & 97.38 & 70.96 & 95.24 & 99.48 & 97.35 & 98.79 & 77.48 & 99.89 \\
\hline Eq. 3 & 96.58 & 74.34 & 92.02 & 99.20 & 94.86 & 98.92 & 74.22 & 99.79 \\
\hline Eq. 4 & 98.28 & 61.63 & 97.01 & 99.61 & 99.39 & 98.95 & 80.15 & 99.90 \\
\hline Eq. 5 & 99.11 & 82.90 & 97.62 & 99.73 & 98.46 & 99.43 & 87.08 & 99.90 \\
\hline Eq. 6 & 98.86 & 79.13 & 94.85 & 98.99 & 98.13 & 98.79 & 74.50 & 98.87 \\
\hline Eq. 7 & 99.09 & 78.61 & 96.90 & 99.42 & 98.50 & 99.04 & 69.53 & 99.67 \\
\hline Eq. 8 & 99.50 & 81.94 & 95.61 & 99.43 & 99.89 & 99.46 & 52.32 & 96.24 \\
\hline Eq. 9 & 99.34 & 86.02 & 94.58 & 99.04 & 99.89 & 99.24 & 70.21 & 93.55 \\
\hline Eq. 10 & 99.20 & 66.30 & 94.57 & 99.41 & 99.99 & 99.21 & 74.48 & 98.52 \\
\hline Eq. 11 & 98.25 & 90.29 & 96.10 & 99.86 & 97.94 & 99.84 & 61.32 & 99.41 \\
\hline Eq. 12 & 99.33 & 87.31 & 95.32 & 99.42 & 99.77 & 99.52 & 60.03 & 93.73 \\
\hline Eq. 13 & 99.71 & 92.38 & 95.74 & 99.35 & 99.77 & 99.33 & 71.90 & 96.70 \\
\hline Eq. 14 & 98.03 & 79.89 & 96.05 & 99.78 & 94.95 & 99.63 & 61.54 & 98.15 \\
\hline Eq. 15 & 98.20 & 91.50 & 96.69 & 99.51 & 90.68 & 99.41 & 68.49 & 99.12 \\
\hline
\end{tabular}

${ }^{\mathrm{a}} \Delta \mathrm{P} \%=\left[\mathrm{P}(\right.$ Active $)-\mathrm{P}($ Inactive) $] x 100$ of each compounds in this screening set (see experimental section). Classification of each compounds using every obtained $\boldsymbol{C}_{\boldsymbol{I}}$ models in the following order: Eqs. 1-15. Here, to consider every query molecule as active chemical, we used $\Delta \mathrm{P} \%>50 \%$ (by $70 \%$ of models), because with this cut-off we avoid the unclassified example as well as the risk that false active can be less. Carbamazepine (CBZ). 
Table 5. Activity and toxicity of substances tested in anti-inflammatory assay in zebrafish.

\begin{tabular}{|c|c|c|c|c|c|}
\hline \multirow{2}{*}{ Compound } & \multicolumn{4}{|c|}{$\begin{array}{c}\text { Relative leukocyte migration (RLM) } \\
\text { Concentration }(\mu M)\end{array}$} & \multirow[t]{2}{*}{$\begin{array}{r}\text { MTC }^{\mathrm{a}} \\
(\mu \mathrm{M})\end{array}$} \\
\hline & 3 & 10 & 30 & 100 & \\
\hline Bisacodyl 1 & - & $0.44 \pm 0.02$ & $0.11 \pm 0.03$ & $\mathrm{~T}^{\mathrm{b}}$ & $30^{*}$ \\
\hline Carbamazepine & - & - & - & $0.60 \pm 0.04$ & $>100$ \\
\hline Dicumarol & $0.45 \pm 0.01$ & $\mathrm{~T}$ & $\mathrm{~T}$ & $\mathrm{~T}$ & $3 *$ \\
\hline Mepartricin & $0.71 \pm 0.01$ & $\mathrm{~T}$ & $\mathrm{~T}$ & $\mathrm{~T}$ & $3 *$ \\
\hline Phenolphthalein & - & - & - & $0.48 \pm 0.05$ & $>100$ \\
\hline Protriptyline hydrochloride & - & - & $0.67 \pm 0.10$ & $\mathrm{~T}$ & $30^{*}$ \\
\hline
\end{tabular}

${ }^{\mathrm{a}} \mathrm{MTC}=$ Maximum Tolerated Concentration. ${ }^{\mathrm{b}} \mathrm{T}=$ Toxic concentration for zebrafish. $*$ Maximum concentration tested due to problems of solubility in Danieau's medium.

\subsection{Proof-of-Concept: In Vivo Evaluation}

Danio rerio (zebrafish) has become a widely used model organism because of its fecundity, its morphological and physiological similarity to mammals, the existence of many genomic tools and the ease with which large, phenotypebased screens can be performed [64].

On the contrary of enzymatic assays, in vivo animal models offer the possibility to screen for biomedical relevant bioactivities in normal condition [65]. In this regard, the amenability of using zebrafish embryos and larvae allows early in vivo analysis of chemically synthesized and naturally derived small-molecule compounds using microgram scale in dependence of the potency of compounds [66]. Also, the possibility of rapid screening represents another excellent benefit of using zebrafish as a model organism over other higher vertebrates [50,67]. In the case of LPS-enhanced leukocyte migration assay, the recruitment of heterophils to a site of injury performed by a transaction of the tail in zebrafish larvae is induced [50]. This phenomenon is representative for blood cells involved in inflammatory processes, which move about to the damage zone $[68,69]$.

Before running the test, the toxicological evaluation at dose of assay was carried out for eight compounds selected with potential anti-inflammatory activity by LBVS, namely Carbamazepine (CBZ), Cyclocumarol (CCL), Dicumarol (DCL), Mepartricin (MPT), Phenolphthalein (PPT), Protriptyline hydrochloride (PTH) and Tetrandrine (TTD). In this sense, the substances were tested at dose where no changes in the normal morphology and physiology of larvae, pericardial oedema, normal posture and death were observed. The relative leukocyte migration (RLM) to the injury zone is shown in Table 5.

The observed RLM for the positive control indomethacin at $30 \mu \mathrm{M}(0.15)$ is in correspondence with potent antiinflammatory activity of this drug. This drug inhibits the cyclooxygenases (COX), enzymes responsible of prostaglandine $(\mathrm{PG})$ production involved in inflammatory process- es. Analyzing the RLM for each substances, it is appreciable that BSL at $30 \mu \mathrm{M}$ showed the best result with 0.11 , indicating an anti-inflammatory activity of $89 \%$ (anti-inflammatory activity is indicated by the formule: $(1-\mathrm{RLM}) * 100$. This result is superior to the value obtained by positive control $(85 \%)$.

Having into account the result of this test, BSL was selected to be evaluated in other in vivo acute antiinflammatory assays including rodents (Fig. 2).

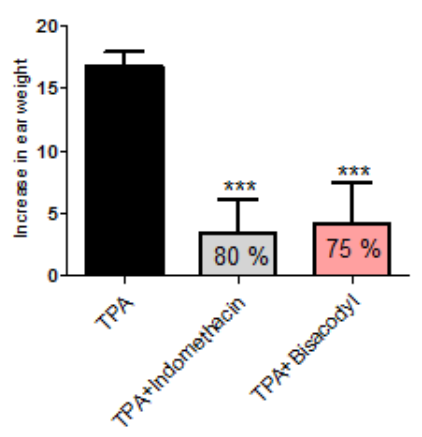

A

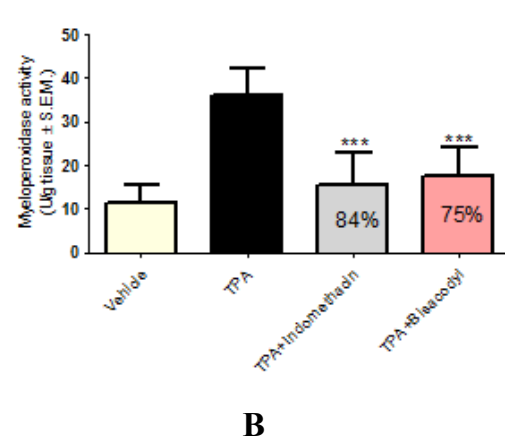

Fig. (2). A (top): Effects of BSL on TPA-induced mouse ear oedema. *** $\mathrm{P}$ $<0.01$; B (bottom): Effects of BSL on changes in MPO activity in TPAinduced mouse ear oedema. $* * * \mathrm{P}<0.001$. vs TPA-treated group. Values inside boxes represent percent inhibition. 
Table 6. Anti-inflammatory activity of Bisacodyl in the Carrageenan-induced paw oedema test in rats.

\begin{tabular}{|c|c|c|c|c|c|c|}
\hline \multirow{2}{*}{ Compound } & \multirow{2}{*}{$\begin{array}{c}\text { Dose } \\
\text { (mg/kg) }\end{array}$} & \multicolumn{5}{|c|}{ Oedema size (\% inhibition) } \\
\hline & & $1 \mathrm{~h}$ & $2 \mathrm{~h}$ & $3 \mathrm{~h}$ & $4 \mathrm{~h}$ & $5 \mathbf{h}$ \\
\hline None & - & $0.28 \pm 0.14$ & $0.47 \pm 0.21$ & $0.73 \pm 0.17$ & $0.79 \pm 0.28$ & $0.77 \pm 0.29$ \\
\hline \multirow{2}{*}{ Indomethacin } & \multirow{2}{*}{10} & $0.22 \pm 0.06$ & $0.35 \pm 0.13$ & $0.45 \pm 0.13^{*}$ & $0.49 \pm 0.08^{*}$ & $0.40 \pm 0.10^{*}$ \\
\hline & & $(22.40)$ & $(26.50)$ & $(38.52)$ & $(37.84)$ & $(47.70)$ \\
\hline \multirow{6}{*}{ Bisacodyl 1} & \multirow{2}{*}{10} & $0.15 \pm 0.06$ & $0.50 \pm 0.10$ & $0.65 \pm 0.06$ & $0.61 \pm 0.12 *$ & $0.40 \pm 0.16^{*}$ \\
\hline & & $(45.38)$ & ( NI ) & $(10.45)$ & (22.07) & $(47.99)$ \\
\hline & \multirow{2}{*}{20} & $0.16 \pm 0.07$ & $0.31 \pm 0.09$ & $0.46 \pm 0.10^{*}$ & $0.43 \pm 0.10^{*}$ & $0.38 \pm 0.10^{*}$ \\
\hline & & $(43.22)$ & $(35.10)$ & $(37.53)$ & $(45.26)$ & $(50.43)$ \\
\hline & \multirow{2}{*}{40} & $0.10 \pm 0.04 *$ & $0.18 \pm 0.01 *$ & $0.20 \pm 0.05^{*}$ & $0.21 \pm 0.06^{*}$ & $0.17 \pm 0.01 *$ \\
\hline & & $(65.23)$ & $(61.72)$ & $(72.23)$ & $(73.48)$ & (77.99) \\
\hline
\end{tabular}

${ }^{a}$ Each value represents the mean $\pm \mathrm{SD}(\mathrm{n}=6)$. Significance levels ${ }^{*} p<0.05$ as compared with the respective control. (ANOVA One way. Post Hoc Test-Tukey's Test). Values in parenthesis represent percent inhibition of oedema. NI: non inhibition. * Maximum concentration tested due to problems of solubility in Danieau's medium.

The TPA-induced mouse ear oedema model is useful for screening prospective topical anti-inflammatory compounds or botanical extracts that act at a variety of levels. TPA applied topically to mouse ears promotes mast cell infiltration with release of mediators that increase vascular permeability and promote neutrophil influx [70]. Products of arachidonic acid metabolism such as PGI2 and leucotriene (LT)B4 increase vascular permeability leading to oedema during the inflammatory response, and compounds inhibiting COX and LOX enzymes have been shown to inhibit TPA induced inflammation [71,72]. An early hallmark of skin irritation and local inflammation in the TPA model is thickening within 1$4 \mathrm{~h}$ due to increased vascular permeability, oedema and swelling within the dermis. Topical application of BSL $(0.5$ $\mathrm{mg} / \mathrm{ear}$ ) significantly inhibited ear oedema at $4 \mathrm{~h}$ after TPA treatment respect to control group, vehicle-TPA (Fig. 2A). Secondarily, polymorphonuclear leukocytes migrate to the dermis at $4 \mathrm{~h}$ was estimated by the myeloperoxidase assay. In this case, $75 \%$ of MPO activity inhibition was obtained (Fig. 2B). BSL reduced oedema and inhibited leukocyte infiltration comparable to indomethacin $(0.5 \mathrm{mg} / \mathrm{ear})$. Considering all results, the anti-inflammatory activity of BSL was evaluated by carrageenan-induced paw oedema test in rats [54]. The administration of a solution of sulfate mucopolysaccharide, extracted of the marine alga Chondrus crispus, in the level of the plantar aponeurosis of the rat, causes a reaction of sharp inflammatory character specific and of twophase type [73]. The initial phase is attributed to the histamine and serotonine liberation while in the second phase, prevails prostaglandins PGE1, PGE2 and PGF2 [74,75]. Oedema maintained between the first and second phase is due to the bradykinin and diverse factors of the complement implied in the inflammation acting as amplifiers of the reponse [76]. Another important mediator is nitric oxide (NO), which is synthesized by constituent and inducible oxide nitric synthase enzymes, in the first phase and second one of the inflammation, respectively [77].

These chemical mediators cause increased vascular permeability, fluid accumulation in tissues and consequently the development of oedema, which is used as variable for the evaluation of the anti-inflammatory activity in the experimental model [54].

The anti-inflammatory activity for BSL is reported in Table 6. The tested compound protected rats from carrageenan-induced inflammation at doses studied. At dose of 20 $\mathrm{mg} / \mathrm{kg}$, the substance showed equipotent anti-inflammatory activity when compared to the indomethacin, while the effect was higher at $40 \mathrm{mg} / \mathrm{kg}$.

\subsection{In Vitro Assays: Searching the Action Modes}

In addition to in vivo assays, some in vitro assays were carried out using murine RAW 264.7 macrophages where BLS at $100 \mu \mathrm{M}$ exhibited no cytotoxicity. Posteriorly, the effect on NO and PGE2 production was determined. At 100 $\mu \mathrm{M}$, a little non significant inhibition of nitrite levels was observed respect to control, (Fig. 3A). For another part, the PGE2 synthesis was not inhibited (Fig. 3B).

Unsatisfactory results were obtained concerning of inducible nitric oxide synthase (iNOS) and cyclooxygenase-2 (COX-2) enzymes expression (Fig. 4).

The data given are the mean \pm S.E.M. of the mean taken from 3 independent experiments for each enzyme. ns $p>0.05$ vs LPS-treated group. The histograms represent the data derived from the Western blots following densitometry analysis. In Fig (4A and 4B), levels were normalized against $\beta$ actin. In Fig. (4A), only a weak diminution of iNOS expression was observed. However, no inhibition effect was observed for COX-2 (Fig. 4B).

Other mediators participating in acute inflammation are cytokines. It is widely recognized that the secretions of cytokines, particularly tumor necrosis factor alpha (TNF- $\alpha$ ) and interleukin (IL) 6, are key mediators of the inflammatory response. In this sense a significance reduction of IL- 6 release was observed in comparison with the group treated with LPS (Table 7). 

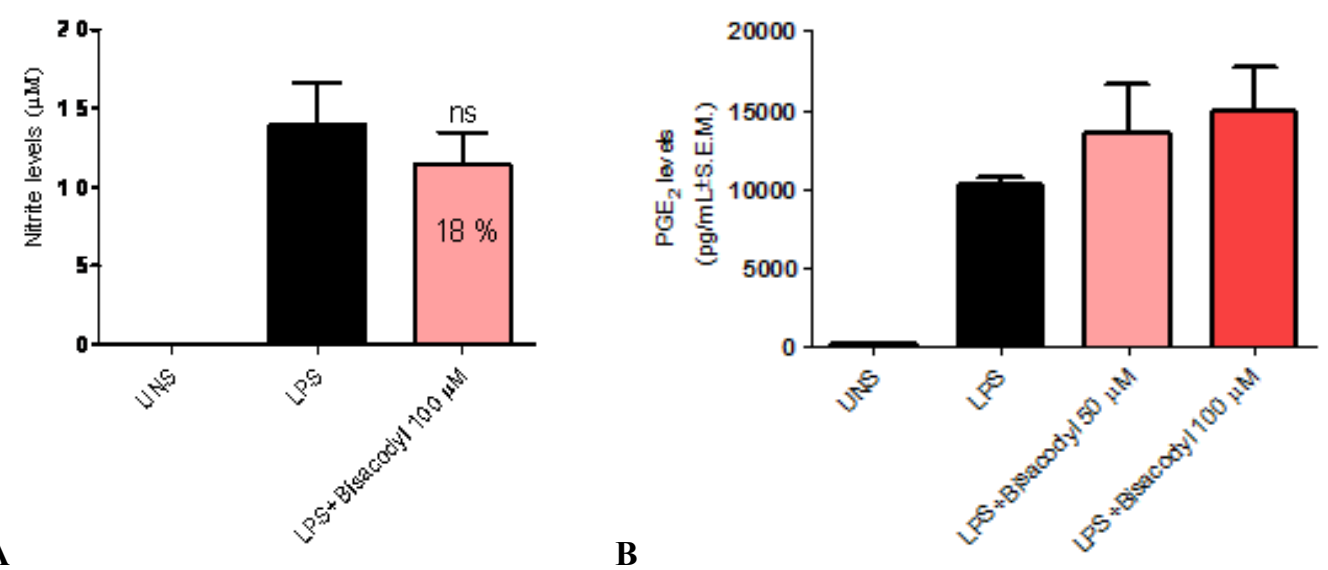

Fig. (3). Effect of BSL 1 on LPS-induced nitrite production in RAW 264.7 cells (A, Left; ${ }^{\mathrm{ns}} \mathrm{p}>0.05$ vs.) and on PGE 2 production in LPSstimulated RAW 264.7 (B, right). UNS: no substance.

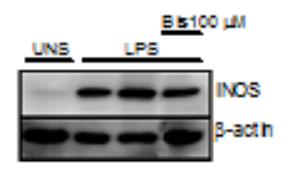

$\mathbf{A}$

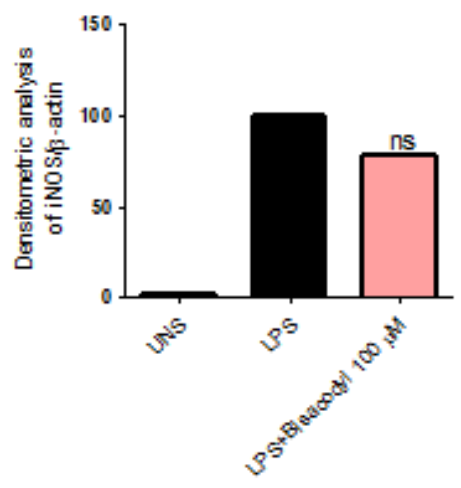

B
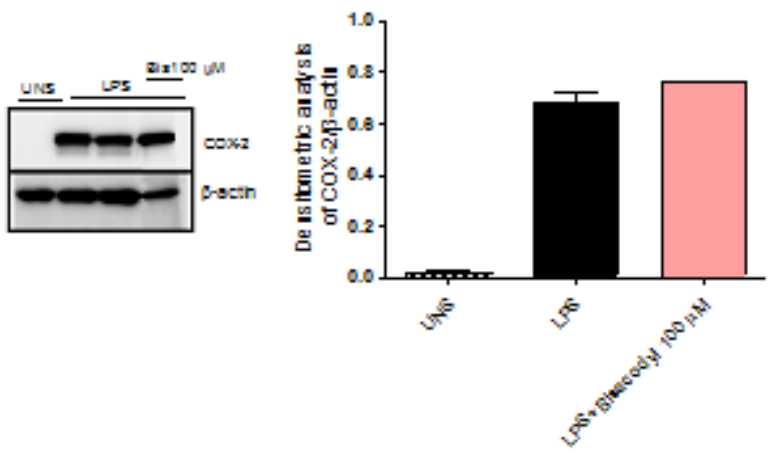

Fig. (4). Effect of $100 \mu \mathrm{M}$ BSL treatment on levels of A (left): iNOS and B (right): COX-2 in RAW 264.7 macrophages. UNS: no substance.

Table 7. Inhibitory effect of Bisacodyl on TNF- $\alpha$ and IL-6 release in RAW264.7 cells.

\begin{tabular}{|c|c|c|}
\hline Treatment & TNF- $\boldsymbol{\alpha}(\mathbf{p g} / \mathbf{m L})$ & IL-6 $^{(\mathbf{n g} / \mathbf{m L})}$ \\
\hline \hline UNS $^{\mathrm{a}}$ & $2143.13 \pm 1137.91$ & $21.53 \pm 1.67$ \\
\hline LPS & $74386.84 \pm 3343.84$ & $2310.52 \pm 42.91$ \\
\hline Bisacodyl & $78639.94 \pm 9060.81$ & $1107.99 \pm 291.97 * *$ \\
\hline
\end{tabular}

Values are expressed as the mean \pm SEM. Significance was determined by ANOVA followed by Dunnett's test.** $p<0.01 v s$. LPS group. ${ }^{a} \mathrm{UNS}$ : no substance.

Considering all biological results, we could suggest that anti-inflammatory activity of Bisacodyl observed in vivo assays may be related to the release of cytokines in particulary with IL-6. Another possibility to explore in the future is the 5-lypoxygenase pathway.

\subsection{Chemistry}

Taking into account the results obtained in the evaluation of the anti-inflammatory activity of BSL, we decided to synthesize some analogues to evaluate their anti-inflammatory potential.
Compounds prepared for this study are shown in Scheme 1 and Table 8. The synthesis of the diarylmethylpyridines $N$ oxides 7 was carried out following the synthetic pathways represented in Scheme 1. First, synthesis of corresponding diarylmethyl-pyridines has been achieved by Friedel-Crafts hydroxy-alkylation reactions of 2-pyridinecarboxaldehyde $\mathbf{2}$ with aromatic compounds under superelectrophilic activation using a mixture of TFSA and TFA. The $p, p$ regioisomers were obtained in a range of 37 to $78 \%$ yield in high purity (HPLC, generally $>92 \%$ ). The formation of $o, p$ regioisomers 5 was observed and, when possible, they were isolated and caracterized. In some cases the synthesis of corresponding diarylmethylpyridines was accomplished using concentrated sulfuric (compounds $\mathbf{4 e}$ and $\mathbf{4 f}$ ) or chlorhydric acid (compound $\mathbf{4 g}$ ) as brønsted acids. In general, the reaction was very simple and clean, avoiding the use of organic solvents and other expensive acidic systems. Compound 6 was synthesized from compound $\mathbf{4 g}$, previously obtained by Friedel-Crafts reaction as reported in Scheme 1.

During the oxidation of the bis (4-acetylaminophenyl)-2pyridylmethane 6 and the BLS the formation of some byproducts was observed. These compounds were isolated and characterized. Compound $\mathbf{1 1}$ was prepared starting from 9, by amidolysis in $1.2 \mathrm{M} \mathrm{HCl}$ in $85 \%$ yield. 


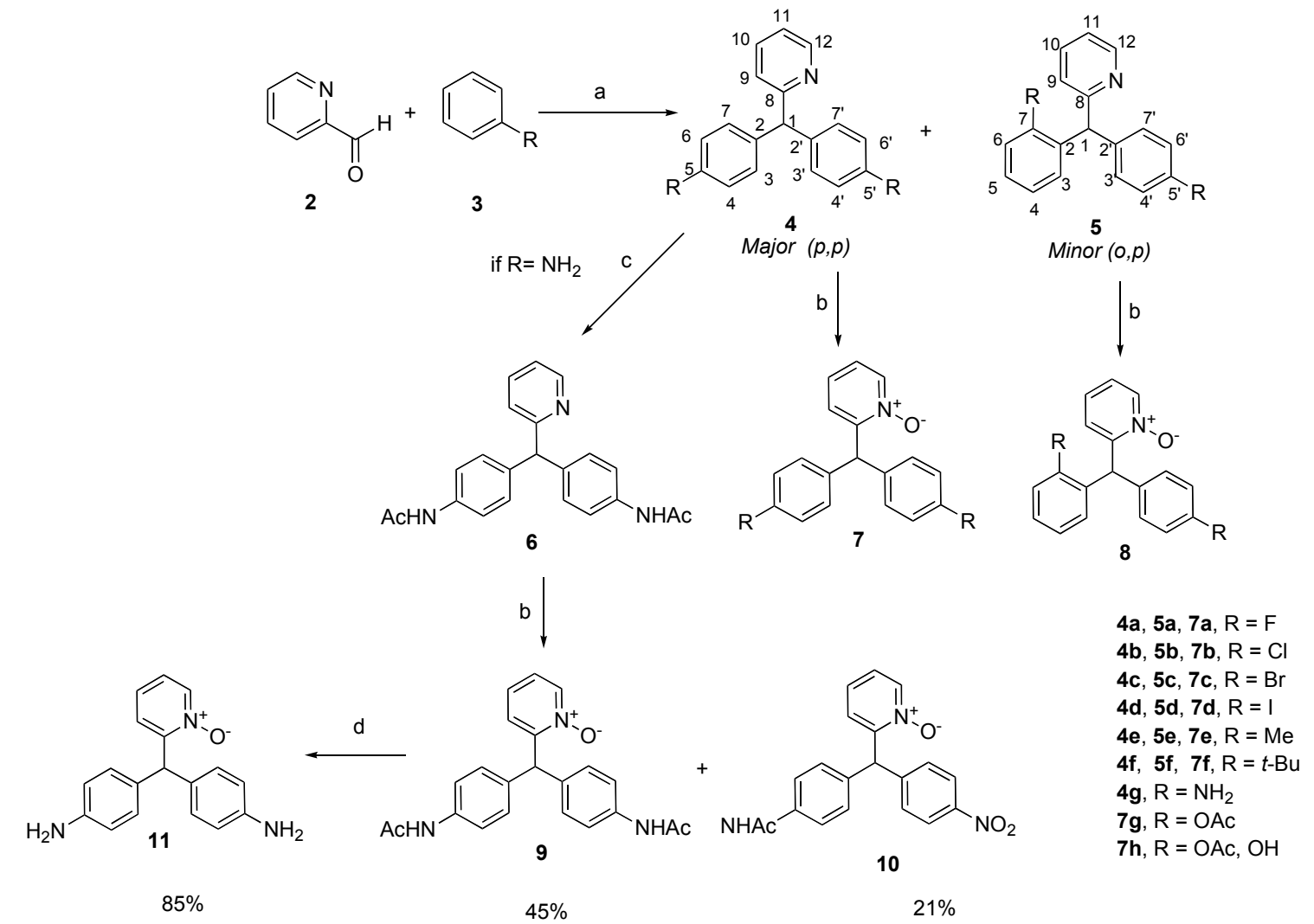

Scheme 1. Reagent and conditions: (a) for $\mathrm{R}=\mathrm{F}, \mathrm{Cl}$, Br, I: TFSA/TFA (5:15), rt; for $\mathrm{R}=\mathrm{CH}_{3}$ or tert-Bu, $\mathrm{conc}_{2} \mathrm{H}_{2} \mathrm{SO}_{4}, \mathrm{RT}_{\text {, for } \mathrm{R}=\mathrm{NH}}$, $\mathrm{HCl}$, $150^{\circ} \mathrm{C}$; (b) $\mathrm{H}_{2} \mathrm{O}_{2} 35 \% 5$ equiv, $\mathrm{AcOH}$; (c) $\mathrm{Ac}_{2} \mathrm{O}, \mathrm{CH}_{2} \mathrm{Cl}_{2}$, $\mathrm{RT}$; (d) $\mathrm{HCl}, 1.2 \mathrm{M}$, reflux.

\subsection{In Vivo Wet Evaluation of Bisacodyl Analogues}

Compounds prepared in this study were evaluated in vivo, using LPS-enhanced leukocyte migration assay in zebrafish. The anti-inflammatory activity was evaluated according to the extent of leukocyte migration which was expressed by the relative leukocyte migration (RLM, $\mu \mathrm{M})$ to a site of injury. In this assay, we considered RLM values $\leq 0.5$ to represent significant anti-inflammatory activity. The MTC were also determined. The results obtained for 23 BSL analogues in the experimental assays (wet evaluation) are described in Table 8.

Based on the above-mentioned BSL properties, we used this known drug to develop novel anti-inflammatory agents with improved anti-inflammatory potency and pharmacological profile and reduced side effects. On these bases, our design strategy focused on the preparation of diarylmethylpyridines bearing a pyridine $N$-oxide moiety. It has been described in the literature that the introduction of a pyridine $N$ oxide group may play an important role in the biological activity. This function could increase the resistant to oxidation enhancing the metabolic stability or increase the bioavailability by improving the pharmacokinetic parameters. Sometimes, pyridine $N$-oxide group increases the affinity for the receptor site via the formation of hydrogen bonds. Further, the activity of certain prodrugs bearing pyridine ring, is largely attributed to their $N$-oxide metabolites formed in vivo. [78-82] In this study, for comparison, when possible, we evaluated the anti-inflammatory activity of pyridine derivatives and their corresponding $N$-oxides.
The structure-activity study showed that the introduction of a bulky alkyl substituent such as tert-butyl group was disadvantageous to anti-inflammatory activity as demonstrated by compound $4 \mathbf{f}$ which displayed RLM values of 0.86 at 10 $\mu \mathrm{M}$. Similarly, the $N$-oxide analogue $7 \mathbf{f}$ was found to be inactive with RLM values of 0.95 at $3 \mu \mathrm{M}$ corresponding to a low inhibition $(5 \%)$ of migrating leukocytes. In addition, this compound exhibited solubility problems during biological studies. In contrast, activity increased dramatically when a methyl group was introduced. Compound 4e showed an activity comparable with indomethacin with RLM values of 0.48 and 0.31 when tested at 3 and $10 \mu \mathrm{M}$, respectively.

When a pyridine oxide moiety was introduced, the resulting compound 7e was substantially more active than its counterpart displaying RLM values of $0.16,0.14$ and 0.10 at 3,10 and $30 \mu \mathrm{M}$, respectively. These results suggested that in the case of alkyl substituted diarylmethylpyridines, the introduction of the $\mathrm{N}$-oxide moiety could play an important role in the anti-inflammatory activity.

In the halogenated pyridyl series, whereas fluoro, chloro, bromo and iodo $p$, $p$-regioisomers derivatives $4 \mathbf{a}, \mathbf{4 b}, \mathbf{4 c}$ and 4d respectively, were equipotent (RLMs of $0.17,0.14,0.13$ and 0.11 at $10 \mu \mathrm{M}$ ), the corresponding $N$-oxide compounds 7a, 7b, 7c and 7d were less potent (RLMs of $0.31,0.48,0.25$ and 0.23 respectively at $10 \mu \mathrm{M}$ ). The same behaviour was observed for these compounds when tested at $3 \mu \mathrm{M}$. These observations could indicate that electronic and/or hydrophobic effects may be at play in the SAR of the diarylmethylpyridines analogues. On the other hand, both fluorine pyridyl 
Table 8. Anti-inflammatory activity (RLM assay) and toxicity (MTD) of BSL analogues 4-11 tested in zebrafish.

\begin{tabular}{|c|c|c|c|c|c|c|c|c|c|}
\hline \multicolumn{4}{|c|}{ Compound Substituant } & \multicolumn{5}{|c|}{ RLM at various concentrations $(\mu \mathrm{M})^{\mathbf{a}}$} & \multirow{2}{*}{$\begin{array}{l}\text { MTC } \\
(\mu \mathrm{M})\end{array}$} \\
\hline $\mathbf{N}^{\circ}$ & $\mathbf{R}_{1}$ & $\mathbf{R}_{2}$ & $\mathbf{R}_{3}$ & 1 & 3 & 10 & 30 & 100 & \\
\hline $4 a$ & - & $p-\mathrm{F}$ & $\mathrm{F}$ & - & $0.17 \pm 0.03$ & $0.17 \pm 0.00$ & $0.19 \pm 0.04$ & $\mathrm{~T}$ & 30 \\
\hline $4 b$ & - & $p-\mathrm{Cl}$ & $\mathrm{Cl}$ & $0.50 \pm 0.08$ & $0.45 \pm 0.03$ & $0.14 \pm 0.01$ & $\mathrm{~T}$ & $\mathrm{~T}$ & 10 \\
\hline $5 \mathbf{b}$ & - & $o-\mathrm{Cl}$ & $\mathrm{Cl}$ & $0.57 \pm 0.21$ & $0.22 \pm 0.11$ & $0.14 \pm 0.03$ & $\mathrm{~T}$ & $\mathrm{~T}$ & 10 \\
\hline $4 c$ & - & $p-\mathrm{Br}$ & $\mathrm{Br}$ & $0.24 \pm 0.11$ & $0.13 \pm 0.05$ & $0.13 \pm 0.05$ & $\mathrm{~T}$ & $\mathrm{~T}$ & 10 \\
\hline $5 c$ & - & $o-\mathrm{Br}$ & $\mathrm{Br}$ & $0.32 \pm 0.11$ & $0.16 \pm 0.02$ & $0.08 \pm 0.03$ & $\mathrm{~T}$ & $\mathrm{~T}$ & 10 \\
\hline $4 d$ & - & $p$-I & I & $0.43 \pm 0.25$ & $0.15 \pm 0.08$ & $0.11 \pm 0.04$ & - & - & $10^{*}$ \\
\hline $5 d$ & - & $o-\mathrm{I}$ & I & $0.52 \pm 0.18$ & $0.33 \pm 0.02$ & $0.12 \pm 0.00$ & - & - & $10^{*}$ \\
\hline $4 e$ & - & $p$-Me & $\mathrm{Me}$ & $0.54 \pm 0.02$ & $0.48 \pm 0.02$ & $0.31 \pm 0.13$ & $\mathrm{~T}$ & $\mathrm{~T}$ & 10 \\
\hline $4 f$ & - & $p-t-\mathrm{Bu}$ & $t-\mathrm{Bu}$ & - & - & $0.86 \pm 0.08$ & $0.82 \pm 0.10$ & $0.80 \pm 0.07$ & $>100$ \\
\hline $4 \mathrm{~g}$ & - & $p-\mathrm{NH}_{2}$ & $\mathrm{NH}_{2}$ & - & - & $0.73 \pm 0.07$ & $0.73 \pm 0.07$ & $0.50 \pm 0.03$ & $>100$ \\
\hline 6 & - & $p$-NHAc & NHAc & - & - & $0.64 \pm 0.06$ & $0.62 \pm 0.07$ & $0.62 \pm 0.04$ & $>100$ \\
\hline $7 \mathbf{a}$ & $\mathrm{O}^{-}$ & $p-\mathrm{F}$ & $\mathrm{F}$ & - & - & $0.31 \pm 0.07$ & $0.28 \pm 0.09$ & $0.14 \pm 0.07$ & $>100$ \\
\hline $7 b$ & $\mathrm{O}^{-}$ & $p-\mathrm{Cl}$ & $\mathrm{Cl}$ & - & $0.50 \pm 0.02$ & $0.48 \pm 0.03$ & $0.24 \pm 0.05$ & $\mathrm{~T}$ & 30 \\
\hline $7 \mathrm{c}$ & $\mathrm{O}^{-}$ & $p-\mathrm{Br}$ & $\mathrm{Br}$ & $0.64 \pm 0.13$ & $0.42 \pm 0.04$ & $0.25 \pm 0.11$ & $\mathrm{~T}$ & $\mathrm{~T}$ & 10 \\
\hline $7 d$ & $\mathrm{O}^{-}$ & $p$-I & I & - & $0.21 \pm 0.01$ & $0.23 \pm 0.01$ & $0.22 \pm 0.05$ & - & $30^{*}$ \\
\hline $7 e$ & $\mathrm{O}^{-}$ & $p-\mathrm{Me}$ & $\mathrm{Me}$ & - & $0.16 \pm 0.02$ & $0.14 \pm 0.00$ & $0.10 \pm 0.02$ & $\mathrm{~T}$ & 30 \\
\hline $7 f$ & $\mathrm{O}^{-}$ & $p-t-\mathrm{Bu}$ & $t-\mathrm{Bu}$ & $1.00 \pm 0.06$ & $0.95 \pm 0.05$ & - & - & - & $10^{*}$ \\
\hline 11 & $\mathrm{O}^{-}$ & $p-\mathrm{NH}_{2}$ & $\mathrm{NH}_{2}$ & - & - & $0.49 \pm 0.10$ & $0.36 \pm 0.09$ & $0.16 \pm 0.02$ & $>100$ \\
\hline 9 & $\mathrm{O}^{-}$ & $p$-NHAc & NHAc & - & - & $0.57 \pm 0.02$ & $0.51 \pm 0.01$ & $0.49 \pm 0.04$ & $>100$ \\
\hline $7 g$ & $\mathrm{O}^{-}$ & $p$-OAc & OAc & - & - & $0.38 \pm 0.00$ & $0.43 \pm 0.04$ & $0.37 \pm 0.01$ & $>100$ \\
\hline 10 & $\mathrm{O}^{-}$ & $p$-NHAc & $\mathrm{NO}_{2}$ & - & - & $0.60 \pm 0.24$ & $0.47 \pm 0.06$ & $0.61 \pm 0.26$ & $>100$ \\
\hline $7 \mathrm{~h}$ & $\mathrm{O}^{-}$ & $p$-AcO & $\mathrm{OH}$ & - & - & $0.47 \pm 0.03$ & $0.28 \pm 0.03$ & $0.22 \pm 0.10$ & $>100$ \\
\hline $8 b$ & $\mathrm{O}^{-}$ & $o-\mathrm{Cl}$ & $\mathrm{Cl}$ & - & $0.48 \pm 0.07$ & $0.29 \pm 0.03$ & $0.22 \pm 0.04$ & $\mathrm{~T}$ & 30 \\
\hline \multicolumn{4}{|c|}{ Indomethacin } & $0.50 \pm 0.01$ & $0.37 \pm 0.00$ & $0.20 \pm 0.01$ & $0.15 \pm 0.02$ & $0.13 \pm 0.01$ & $>100$ \\
\hline
\end{tabular}

Values are expressed as the mean $\pm \mathrm{SEM} . \mathrm{T}=$ Toxic concentration for zebrafish. *Maximum concentration tested due to problems of solubility in Danieau's medium.

compound 4a and $N$-oxide derivative 7a showed low toxicity compared with other halogenated compounds (MTC 30 and $>100 \mu \mathrm{M}$ respectively).

In a similar manner, in the halogenated pyridyl series, the chloro and iodo $o, p$-regioisomers $\mathbf{5 b}$ and $\mathbf{5 d}$, showed comparable activities with RLMs of 0.14 and 0.12 respectively at $10 \mu \mathrm{M}$ in comparison with the corresponding $p$, $p$-analogues 4b and 4d. This could mean that, the introduction of a halogen atom either at the para or ortho position of one phenyl ring did not significantly alter the anti-inflammatory activity. In contrast, the insertion of a bromo function at the ortho position leading to the $o, p$ regioisomer $\mathbf{5 c}$, led to a dramatic improvement of biological activity (RLM of 0.08 at $10 \mu \mathrm{M}$ ).

On the other hand, the chloro $o, p$ regioisomer $N$-oxide $\mathbf{8 b}$ showed good activities, comparable to the indomethacin, albeit weaker than the pyridyl analogue $\mathbf{5 b}$. The chloro compound $o, p N$-oxide $\mathbf{8 b}$ displayed RLM values of 0.48 and 0.29 at 3 and $10 \mu \mathrm{M}$, respectively while chloro $o, p$ substituted pyridyl analogue 5b showed RLM values of 0.22 and 0.14 respectively, at the same concentrations.

The pyridine amino derivative, compound 4g was substantially less active than the corresponding counterpart compound $N$-oxide 11. The compound 4g displayed RLM values of 0.73 when tested at 10 and $30 \mu \mathrm{M}$, and RLM value of 0.5 at $100 \mu \mathrm{M}$. The amino substituted $N$-oxide analogue $4 \mathrm{~g}$ showed RLM values of $0.49,0.36$ and 0.16 respectively, at the same concentration. These results suggested once more the influence of the $\mathrm{N}$-oxide moiety in the anti-inflammatory activity. 
Reducing the basicity of the amino function by forming acetamide 6 resulted in greatly increased activity, i.e. at 10, 30 and $100 \mu \mathrm{M}$ compound $\mathbf{6}$ exhibited RLM values around of 0.62 compared with compound $4 \mathrm{~g}$. (RLM of 0.73 ). Moreover, as it was previously observed for the amino derivative, the acetamide substituted compound bearing the pyridine $\mathrm{N}$ oxide function was substantially more active than its pyridyl analogue. However, the introduction of a nitro function, compound 10, obtained following the oxidation of acetamide 6 did not alter significantly the anti-inflammatory activity (RLM value of $0.60,0.47$ and 0.61 at 10,30 and $100 \mu \mathrm{M}$, respectively).

At this purpose, it has to be underlined that electrondonating or -withdrawing groups and steric interactions may significantly modulate the reactivity of diarylmethylpyridines and, consequently, the pharmacological properties. In fact, while the substituents such as methyl or halogen displayed an anti-inflammatory activity comparable with indomethacin, a decrease of activity was observed when they were replaced by the electron-donating groups such as amino and acetamide or by bulky functions like tert-butyl. However, these latter compounds proved to be less toxic (MTC > $100 \mu \mathrm{M})$.

When BSL was oxidized, the resulting compound $\mathbf{7 g}$ showed a slight improvement in the anti-inflammatory activity when tested at $10 \mu \mathrm{M}$ (RLM of $0.38 v s$ RLM of 0.44 for BSL). Nonetheless, a different result was observed when the $N$-oxide analogue was tested at $30 \mu \mathrm{M}$ (RLM of $0.43 v s$ RLM of 0.11 for BSL). It should be noted that despite this decrease in activity, the introduction of an oxide function decreases toxicity of BSL. The monohydroxyl $\mathrm{N}$-oxide analogue compound $\mathbf{7 h}$ was less active than the lead compound but also less toxic.

\section{CONCLUSION}

This report led to the discovery of potent antiinflammatory agents structurally related to Bisacodyl, a known drug currently used in therapy for its laxative properties. The design strategy was focused foremost on exploration the potential of TOMOCOMD-CARDD methods to discover anti-inflammatory drug-like compounds from a heterogeneous series of molecules. The theoretical models obtained here, were used to identify the first anti-inflammatory lead-like by using LBVS of commercially drugs. The in silico prediction was confirmed experimentally in different biological tests.

The second part of our research focused on the development of polyaromatic compounds analogues of BSL with high anti-inflammatory activity. Exploiting this new pharmacophore, potential anti-inflammatories were synthesized, characterized and evaluated in vivo, using LPS-enhanced leukocyte migration assay in zebrafish.

In summary, various BSL derivatives and their corresponding $N$-oxides have been synthesized by Friedel-Crafts hydroxylalkylation with no more than two steps from the corresponding commercially available reagents. The development of these compounds was characterized by a limited number of synthetic steps and by low cost of goods. These compounds have been screened for their anti-inflammatory activities. Compound $\mathbf{5 c}$ and $\mathbf{7 e}$ exhibited the best antiinflammatory activities at $10 \mu \mathrm{M}$ in the pyridyl series and $N$ oxide respectively. In most series of BSL analogues it was demonstrated that the $N$-oxide functionality generally improved the anti-inflammatory activity and decrease toxicity. Moreover, this function could improve the pharmacokinetics profiles of these compounds.

These encouraging results confirm our enthusiasm to search new functionalized compounds with improved antiinflammatory potential. Eighteen compounds synthesized in this work were not reported so far and the synthesis of a new series of other functionalized derivatives is currently under development in our laboratory and will be reported in due course.

\section{LIST OF ABBREVIATIONS (AND UNITS)}

If abbreviations are used in the text either they should be defined in the text where first used, or a list of abbreviations can be provided.
BSL
$=$ Bisacodyl
CBZ
$=$ Carbamazepine
CCL
= Cyclocumarol
Cy
$=$ Cyclohexane
DCL
$=$ Dicumarol
Equiv
$=$ Equivalent
FCC
$=$ Flash column chromatography
HRMS
$=$ High-resolution mass spectra
Ip
LBVS
$=$ Intraperitoneally
LPS
LRMS
$=$ Ligand-Based Virtual Screening
MD
MPO
MPT
MTC
NS
ppm
p-disubst
$=$ Lipopolysaccharide
PPT
PTH
= Low-resolution mass spectra
$=$ Molecular descriptor
RLM
RT
SS
TFA
TFSA
$=$ Myeloperoxidase
$=$ Mepartricin
TPA
TTD
$=$ Maximum Tolerated Concentration
$=$ Non-stochastic
$=$ Parts per million
$=$ Para-disubstituted
$=$ Phenolphthalein
$=$ Protriptyline hydrochloride
$=$ Relative leukocyte migration
$=$ Room temperature
$=$ Simple Stochastic
$=$ Trifluoroacetic acid
$=$ Trifluoromethanesulfonic acid
$=$ O-tetradecanoylphorbol-13-acetate
$=$ Tetrandrine 
TOMOCOMD-

$\begin{aligned} \text { CARDD }= & \text { TOpological MOlecular COMputational } \\ & \text { Design-Computer-Aided Rational Drug } \\ & \text { Design }\end{aligned}$

UNS $\quad=$ No substance

ETHICS APPROVAL AND CONSENT TO PARTICIPATE

Not applicable.

\section{HUMAN AND ANIMAL RIGHTS}

No Animals/Humans were used for studies that are base of this research.

\section{CONSENT FOR PUBLICATION}

Not applicable.

\section{CONFLICT OF INTEREST}

M.S-IV and Y.M. P. designed experiment, analyzed data and wrote the paper, D.S. M, L. V. M and M. M. accomplished biological experiments, Y. R. G. performed computational studies, V. L performed chemical synthesis. P. A. M de W. and A. D. C. and R. M. G. supervised and analyzed biological studies. V J. A. and F. D. supervised and analyzed chemical characterization. C. F. analyzed chemical data and supervised the project.

All authors contributed to the drafting and revision of the article and approved the final version.

\section{ACKNOWLEDGEMENTS}

Dany Siverio-Mota acknowledges the Laboratory for Molecular Biodiscovery, Department of Pharmaceutical and Pharmacological Sciences, University of Leuven (Belgium) for kind hospitality and VLIR (Vlaamse InterUniversitaire Raad, Flemish Interuniversity Council, Belgium) under the IUC Program VLIR-UCLV for in part financial support of this work. Yovani Marrero Ponce thanks the program 'Estades Temporals per a Investigadors Convidats' for a fellowship to work at Universitat de València, Spain. The spanish Ministry of Science and Innovation (project SAF200910399) and LabEx LERMIT (ANR-10-LABX-33) are also acknowlwdged for the financial support.

Authors thanks Prof. José L. Rios for his availability to experimental tests at Universitat de València and Mrs. Annie Falguières for her technical assistance during HPLC analyses at the Conservatoire national des arts et métiers, Paris, France.

\section{SUPPLEMENTARY MATERIAL}

The complete list of compounds used in training and test sets, the results of the classification according to obtained models, the ensemble algorithm as well as additional NMR analysis is available free of charge via the Internet at http://xxxx.yyyy.zz

Supplementary material is available on the publisher's web site along with the published article.

\section{REFERENCES}

[1] Wermuth, C.G. Selective optimization of side activities: another way for drug discovery. J. Med. Chem., 2004, 47(6), 1303-1314.

[2] Rauwerda, H.; Roos M.; Hertzberger B.O.; Breit T.M. The promise of a virtual lab in drug discovery. Drug Discov. Today, 2006, 11, 228-236.

[3] Ashburn, T.T.; Thor, K.B. Drug repositioning: identifying and developing new uses for existing drugs. Nat. Rev. Drug Discov., 2004, 3, 673-683.

[4] Check, W.A.; Kaliner M.A. Pharmacology and pharmacokinetics of topical corticoid derivatives used for asthma therapy. Am. Rev. Respir. Dis., 1990, 141, 44-51.

[5] Geddes, D.M. Inhaled corticoids: benefit and risks. Thorax, 1992, 47, 404-407.

[6] Schäcke, H.; Döcke, W.D.; Asadullah, K. Mechanisms involved in the side effects of glucocorticoids. Pharmacol. Ther., 2002, 96, 2343.

[7] Yasuhiro, T.; Hioko O.; Hiroshi, T. Glucocorticoid-induced osteoporosis. Biomed. Pharmacother., 2004, 58, 500-504.

[8] DiMasi, J.A.; Hansen, R.W.; Grabowski, H.G.J. The price of innovation: new estimates of drug development costs. Health Econ., 2003, 22, 151-185.

[9] Warne P.; Page C. Is there a best strategy for drug discovery? Drug News Perspect., 2003, 16, 177-182.

[10] Weisman J.L.; Liou, A.P.; Shelat A.A.; Cohen F.E.; Guy R.K.; DeRisi J.L. Searching for new antimalarial therapeutics amongst known drugs. Chem. Biol. Drug Des., 2006, 67, 409-416.

[11] Dakshanamurthy S.; Issa N. T.; Assefnia S.; Seshasayee A.; Peters O.J.; Madhavan S.; Uren A.; Brown M.L.; Byers S. W. Predicting new indications for approved drugs using a proteochemometric method. J. Med. Chem., 2012, 55, 6832-6848.

[12] Seifert, M.H.J.; Wolf, K.; Vitt, D. Virtual high-throughput in silico screening. Biosilico, 2003, 1, 143-149.

[13] Martins Alho, M.A.; Marrero-Ponce, Y.; Barigye, S.J.; MenesesMarcel, A.; Machado Tugores, Y.; Montero-Torres, A.; GomezBarrio, A.; Nogal, J.J.; Garcia-Sanchez, R.N.; Vega, M.C.; Rolon, M.; Martinez-Fernandez, A.R.; Escario, J.A.; Perez-Gimenez, F.; Garcia-Domenech, R. n.; Rivera, N.; Mondragon, R.; Mondragon, M. ; Ibarra-Velarde, F. ; Lopez-Arencibia, A.; Martin-Navarro, C.; Lorenzo-Morales, J.; Cabrera-Serra, M.G.; Pinero, J.; Tytgat, J.; Chicharro, R.; Aran, V.J. Antiprotozoan lead discovery by aligning dry and wet screening: Prediction, synthesis, and biological assay of novel quinoxalinones. Bioorg. Med. Chem., 2014, 22, 15681585.

[14] Duart M.J.; Anton-Fos, G.M.; Aleman, P.A.; Gay-Roig, J.B.; Gonzalez-Rosende, M.E.; Galvez J.; Garcia-Domenech, R. new potential antihistaminic compounds. virtual combinatorial chemistry, computational screening, real synthesis, and pharmacological evaluation. J. Med. Chem., 2005, 48, 1260-1264.

[15] Marrero-Ponce, Y.; Siverio-Mota D.; Gálvez-Llompart M.; Recio, M.C, Giner, R.M.; García-Domènech, R.; Torrens, F.; Arán, V.J., Cordero-Maldonado, M.L.; Esguerra, C.V.; de Witte, P.A.M.; Crawford A.D. Discovery of novel anti-inflammatory drug-like compounds by aligning in silico and In vivo screening: The nitroindazolinone chemotype. Eur. J. Med. Chem., 2011, 46, 57365753 .

[16] Kubinyi, H. Chemical similarity and biological activities. J. Braz. Chem. Soc., 2002, 13, 717-726.

[17] Duart, M. J.; Garcia-Domenech, R.; Galvez, J.; Aleman, P.A.; Martin-Algarra, R.V.; M. Anton-Fos, G. Application of a mathematical topological pattern of antihistaminic activity for the selection of new drug candidates and pharmacology assays. J. Med. Chem., 2006, 49, 3667-3673.

[18] Eckert H.; Bajorath J.; Molecular similarity analysis in virtual screening: foundations, limitations and novel approaches. Drug Discov. Today, 2007, 12, 225-236.

[19] Gálvez-Llompart, M.; Giner, R.M.; Recio, M.C.; Candeletti, S.; Garcia-Domenech, R. Application of molecular topology to the search of novel NSAIDs. Experimental validation of activity. Lett. Drug Des. Disc., 2010, 7, 438-445.

[20] García-Domenech, R.; Gálvez, J.; de Julian-Ortiz, J.V.; Pogliani, L. Some new trends in chemical graph theory. Chem. Rev., 2008, 108, 1127-1169.

[21] Marrero-Ponce, Y.; Romero, V. TOMOCOMD software, Central University of Las Villas (UCLV): TOMOCOMD (TOpological 
MOlecular COMputational Design) for Windows, version 1.0 is a preliminary experimental version; in future a professional version can be obtained upon request to Y. Marrero-Ponce: yovanimp@uclv.edu.cu or ymarrero77@yahoo.es. 2002

[22]. STATISTICA. (Data Analysis Software System) version 6. StatSoft Inc, 2001. www.statsoft.com

[23] van de Waterbeemd H. Discriminant analysis for activity prediction. in: H. van de Waterbeemd (Ed), Chemometric Methods in Molecular Design. VCH Publishers, Weinheim, 1995, pp 265-328.

[24] The Merck Index. In 12 ${ }^{\text {th }}$ ed.; Chapman and Hall: 1996.

[25] M. Negwer. Organic-Chemical Drugs and their Synonyms. Akademie: Berlin, 1987.

[26] Karelson, M. Molecular Descriptors in QSAR/QSPR. John Wiley \& Sons: New York, 2000

[27] Katritzky, A.R.; Gordeeva, E.V. Traditional topological indices vs electronic, geometrical, and combined molecular descriptors in QSAR/QSPR research. J. Chem. Inf. Comput. Sci., 1993, 33, 835857.

[28] Van de Waterbeemd, H.; Costantino, G.; Clementi, S.; Cruciani, G.; Valigi, R. Experimental design in synthesis planning and structure-property correlations. Disjoint principal properties of organic substituents. In Chemometric methods in molecular design, H. van de Waterbeemd, Editor. VCH Publishers: New York, 1995 pp 103112 .

[29] Marrero-Ponce, Y.; Torrens, F.; García-Domenech, R.; OrtegaBroche, S.E.; Romero Zaldivar, V. Novel 2D TOMOCOMDCARDD molecular descriptors: atom-based stochastic and nonstochastic bilinear indices and their QSPR applications. J. Math. Chem., 2008, 44, 650-673.

[30] Marrero-Ponce, Y.; Ortega-Broche, S.E.; Díaz, Y.E. Alvarado, Y.J.; Cubillan, N.; Cardoso, G.C.; Torrens, F.; Pérez-Giménez F. Nucleotide's bilinear indices: Novel bio-macromolecular descriptors for bioinformatics studies of nucleic acids. I. Prediction of paromomycin's affinity constant with HIV-1 [Psi]-RNA packaging region. J. Theor. Biol., 2009, 259, 229-241.

[31] Castillo-Garit, J.A.; Martinez-Santiago O.; Marrero-Ponce Y.; Casañola-Martín G.M.; Torrens F. Atom-based non-stochastic and stochastic bilinear indices: Application to QSPR/QSAR studies of organic compounds. Chem. Phys. Lett., 2008, 464, 107-112.

[32] Marrero-Ponce Y.; Khan M.T.H.; Casañola-Martin G.M.; Ather, A.; Sultankhodzhaev, M.N.; Torrens F.;Rotondo, R. Prediction of tyrosinase inhibition spectra for chemicals using novel atom-based bilinear indices. ChemMedChem, 2007, 2, 449-478.

[33] Castillo-Garit J.A.; Marrero-Ponce, Y.; Torrens F.; Rotondo, R. Atom-based stochastic and non-stochastic 3D-chiral bilinear indices and their applications to central chirality codification. J. Mol. Graph. Model., 2007, 26, 32-47.

[34] Marrero-Ponce Y.; Meneses-Marcel, A.; Castillo-Garit, J.A.; Machado-Tugores, Y.; Escario, Gomez Barrio A.; Montero Pereira D.; Nogal-Ruiz J.J.; Arán, V.J., Martínez-Fernández, A.R.; Torrens, F. Rotondo, R.; Ibarra-Velarde, F.; Alvarado, Y.J., Predicting antitrichomonal activity: A computational screening using atombased bilinear indices and experimental proofs. Bioorg. Med. Chem., 2006, 14, 6502-6524.

[35] Julián-Ortiz, J.V.; Gálvez, J.; Muñoz-Collado, C.; GarcíaDomenech, R.; Gimeneo-Cardona, C. Virtual combinatorial synthesis and computational screening of new potential anti-herpes compounds. J. Med. Chem., 1999, 42, 3308-3314.

[36] Klein, D.J. Graph Theoretically Formulated Electronic-Structure Theory. Internet Electron. J. Mol. Des., 2003, 2, 814-834.

[37] Marrero-Ponce, Y.; Linear Y. Indices of the "Molecular Pseudograph's Atom Adjacency Matrix": Definition, significanceinterpretation and application to QSAR analysis of flavone derivatives as HIV-1 integrase inhibitors. J. Chem. Inform. Model. 2004, 44, 2010-2026.

[38] Marrero-Ponce, Y.; Cabrera, M.A.; Romero, V.; González, D.H.; Torrens, F.A. New topological descriptors based model for predicting intestinal epithelial transport of drugs in caco-2 cell culture. $J$. Pharm. Sci., 2004, 7, 186-199.

[39] Marrero-Ponce, Y.; Castillo-Garit, J.A.; Olazabal, E.; Serrano, H.S.; Morales, A.; Castanedo, N.; Ibarra-Velarde, F.; HuescaGuillen, A.; Sanchez, A. M.; Torrens, F.; Castro, E.A. Atom, atomtype and total molecular linear indices as a promising approach for bioorganic and medicinal chemistry: theoretical and experimental assessment of a novel method for virtual screening and rational de- sign of new lead anthelmintic. Bioorg. Med. Chem., 2005, 13, 1005-1020.

[40] Marrero-Ponce, Y.; Medina-Marrero, R.; Castillo-Garit, J.A.; Romero-Zaldivar, V.; Torrens, F.; Castro, E.A. Protein linear indices of the "macromolecular pseudograph's r-carbon atom adjacency matrix" in bioinformatics. 1. prediction of protein stability effects of a complete set of alanine substitutions in arc repressor. Bioorg. Med. Chem., 2005, 13, 3003-3015.

[41] Marrero-Ponce, Y.; Medina-Marrero, R.; Torrens, F.; Martinez, Y.; Romero-Zaldivar, V.; Castro E.A. Atom, atom-type, and total nonstochastic and stochastic quadratic fingerprints: a promising approach for modeling of antibacterial activity. Bioorg. Med. Chem. 2005, 13, 2881-2899.

[42] Baldi, A.; Dragonetti, E. ; Battista, T.; Groeger, A.M.; Esposito, V.; Baldi, G.; Santini D. Detection of circulating malignant cells by RT-PCR in long-term clinically disease-free i stage melanoma patients. Anticancer Res., 2000, 20, 3923-3928.

[43] Atkinson, A.C. Plots, Transformations and Regression. Clarendon Press: Oxford, 1985.

[44] Papa, E.; Villa, F.; Gramatica, P. Statistically P. validated QSARs, based on theoretical descriptors, for modeling aquatic toxicity of organic chemicals in pimephales promelas (fathead minnow). $J$. Chem. Inf. Model., 2005, 45, 1256-1266.

[45] Prakash, G.K.S.; Paknia, F.; Chacko, S.; Mathew, T. Olah, G.A. Facile synthesis of diarylmethylpyridines/diarylmethylquinolines through superelectrophilic activation of pyridinecarboxaldehydes/ quinolinecarboxaldehydes with boron trifluoride monohydrate. Heterocycles., 2008, 76, 783-799.

[46] Schultz, B.E.; Gheller, S.F.; Muetterties, M.C.; Scott, M.J.; Holm R.H. Molybdenum-mediated oxygen-atom transfer: an improved analog reaction system of the molybdenum oxotransferases. $J . A m$. Chem. Soc., 1993, 115, 2714-2722.

[47] Kim, B.; Chinn, A.J.; Fandrick, D.R.; Senanayake, C.H.; Singer, R.A.; Miller, S.J. Distal stereocontrol using guanidinylated peptides as multifunctional ligands: Desymmetrization of diarylmethanes via Ullman cross-coupling. J. Am. Chem. Soc., 2016, 138, 79397945 .

[48] Maes, J. ; Verlooy, L.; Buenafe, O.E; de Witte, P.A.M.; Esguerra C.V.; Crawford A.D. Evaluation of 14 organic solvents and carriers for screening applications in zebrafish embryos and larvae. PLoS ONE, 2012, 7, e43850.

[49] Karlsson, J.; von Hofsten, J.; Olsson, P. Generating transparent zebrafish: A refined method to improve detection of gene expression during embryonic development. Mar. Biotechnol., 2001, 3, 522-527.

[50] Cordero-Maldonado, M. L.; Siverio-Mota, D.; Vicet-Muro, L.; Wilches- Arizábala I.M.; Esguerra, C.V.; de Witte, P.A.M.; Crawford, A.D. Optimization and pharmacological validation of a leukocyte migration assay in zebrafish larvae for the rapid "In Vivo" bioactivity analysis of anti-inflammatory secondary metabolites. PLOS ONE, 2013, 8, e75404.

[51] Young, J.M.; De Young, L.M. Cutaneous models of inflammation for the evaluation of topical and systemic pharmacological agents. Chang, J.Y. and A.J. Lewis, ed.; New York, New York, USA. Illus. 1989; Vol. 5.

[52] Payá, M.; Ferrándiz M., Sanz, M.J; Bustos, G.; Blasco, R; Ríos, J. L.; Alcaraz, M.J. Study of the antioedema activity of some seaweed and sponge extracts from the Mediterranean coast in mice. Phytother. Res., 1993, 7, 159-162.

[53] Giner, R.M.; Villalba, M.L.; Recio, M.C.; Máñez, S. Cerdá-Nicolás M.; Ríos J.L. Anti-inflammatory glycoterpenoids from Scrophularia auriculata. Eur. J. Pharmacol., 2000, 389, 243-252.

[54] Winter, C.A.; Risley, E.A.; Nuss, N.W. Carrageenin induced oedema in hinh paw of the rats an assay for antiinflammatory drug. Proc. Soc. Exp. Biol. Med., 1962, 111, 544-547.

[55] Tropsha, A.G. Predictive QSAR modeling workflow, model applicability domains, and virtual screening. Curr. Pharm. Des., 2007, 13, 3494-3504.

[56] Golbraikh, A.; Shen M.; Xiao, Z.; Xiao, Y.D.; Lee; K.H. Tropsha, A. Rational selection of training and test sets for the development of validated QSAR models. J. Comput. Aided Mol. Des., 2003, 17, 241-253.

[57] Golbraikh, A.; Tropsha, A. Beware of q2! J. Mol. Graph. Model., 2002, 20, 269-276.

[58] Shen, M.; Xiao, Y.; Golbraikh, A.; Gombar, V.K. Tropsha, A. Development and validation of k-nearest-neighbor QSPR models 
of metabolic stability of drug candidates. J. Med. Chem., 2003, 46, 3013-3020.

[59] Van Damme, S.; Langenaeker, W.; Bultinck, P. Prediction of blood-brain partitioning: A model based on ab initio calculated quantum chemical descriptors. J. Mol. Graphics Model., 2008, 26, 1223-1236.

[60] Van Damme, S.; Bultinck, P., Software news and update a new computer program for QSAR-Analysis: ARTE-QSAR. J. Comput. Chem., 2007, 28, 1924-1928.

[61] Estrada, E.; Uriarte, E.; Montero, A.; Teijeira, M.; Santana, L.; De Clercq, E. A novel approach for virtual screening and rational design of anticancer compounds. J. Med. Chem., 2000, 43, 19751985.

[62] Estrada, E.; Vilar, S.; Uriarte, E; Gutierrez, Y. In Silico studies toward the discovery of new anti-HIV nucleoside compounds with the use of TOPS-MODE and 2D/3D connectivity indices. 1. Pyrimidyl Derivatives. J. Chem. Inf. Comput. Sci., 2002, 42, 1194-1203.

[63] Stoll, R.E.; Blanchard, K.T.; Stoltz, J.H.; Majeska, J.B.; Furst, S. Lilly, P.D.; Mennear, J.H. Phenolphthalein and Bisacodyl: assessment of genotoxic and carcinogenic responses in heterozygous p53 $(+/-)$ mice and syrian hamster embryo (SHE). Assay. Toxicol. Sci., 2006, 90, 440-450.

[64] Zon, L.I.; Peterson R.T. In vivo drug discovery in the zebrafish. Nat. Rev. Drug Discov., 2005, 4, 35-44.

[65] Crawford, A.D.; Esguerra, C.V.; de Witte, P.A.M. Fishing for drugs from nature: Zebrafish as a technology platform for natural product discovery. Planta Med., 2008, 74, 624-632.

[66] Crawford, A.D.; Challal, S.; Buenafe, O.E.; Harvey, A.L.; Esguerra, C.V.; de Witte, P.A.M. Wolfender, J.L. Microgram-scale, In vivo natural product discovery using zebrafish bioassays, UHPLCTOF-MS and microflow NMR: Identification of anticonvulsants in the Philippine medicinal plant Solanum torvum. Planta Med., 2011, 77, 1348-1349.

[67] Delvecchio, C.; Tiefenbach, J.; Krause, H.M. The Zebrafish: A powerful platform for In Vivo, HTS drug discovery. Assay Drug Dev. Tecnh., 2011, 9, 354-361.

[68] Renshaw, S.; Loynes, C.; Trushell, D.; Elworthy, A.; Ingham, P. Whyte, M. A transgenic zebrafish model of neutrophilic inflammation. Blood, 2006, 108, 3976-3978.

[69] Lieschke, G.; Oates, A.; Crowhurst, M.; Ward, A.; Layton, J. Morphologic and functional characterization of granulocytes and macrophages in embryonic and adult zebrafish. Blood, 2001, 98, 30873096.

[70] Opas, E.E.; Bonney, R.J.; Humes, J.L. Prostaglandin and leukotriene synthesis in mouse ears inflamed by arachidonic acid. J. Invest. Dermatol., 1985, 84, 253-256.

[71] Carlson, R.P.; O'Neill-Davis, L.; Chang, J.; Lewis, A.J. Modulation of mouse ear oedema by cyclooxygenase and lipoxygenase inhibitors and other pharmacologic agents. Agents Actions, 1985, 17, 197-204.
[72] Bralley, E.E.; Greenspan, P.; Hargrove, J.L.; Wicker, L.; Hartle, D.K. Topical anti-inflammatory activity of Polygonum cuspidatum extract in the TPA model of mouse ear inflammation. J Neuroinflammation, 2008, 5, 1-7.

[73] Vinegar, R.; Schreiber, W.; Hugo, R. Biphasic development of carrageenan in rats. J. Pharmacol. Exp.Ther., 1969, 166, 96-103.

[74] Dirosa, M.; Giround, J. Studies of the mediators of acute inflammatory response induced in rats in different sites by carrageenan and turpentine. J. Phatol., 1971, 104, 15-29.

[75] Heller, A.; Koch, T.; Schmeck, J.; Acker, V. Lipid mediators in inflammatory disorders. Drugs, 1998, 55, 487-496.

[76] Burch, R.; DeHaas, C. A bradykinin antagonist inhibits carrageenan oedema in rats. Naunyn Schmiedebergs, Arch. Pharmacol., 1990, 342, 189-193.

[77] Salvemini, D.; Seibert, K.; Marino, M.H. PG release, as a consequence of NO-driven COX activation contributes to the proinflammatory effects of NO. Drugs News Perspect., 1996, 4, 204219.

[78] Seto, M.; Aramaki, Y.; Okawa, T.; Miyamoto, N.; Aikawa, K.; Kanzaki, N.; Niwa, S.; Iizawa, Y.; Baba, M.; Shiraishi, M. Orally active CCR5 antagonists as anti-HIV-1 agents: synthesis and biological activity of 1-benzothiepine 1,1-dioxide and 1-benzazepine derivatives containing a tertiary amine moiety. Chem. Pharm. Bull., 2004, 52, 577-590.

[79] Alexander, R.P.; Warrellow, G.J.; Eaton, M.A.; Boyd, E.C.; Head, J.C.; Porter, J.R.; Brown, J.A.; Reuberson, J.T.; Hutchinson, B.; Turner, P.; Boyce, B.; Barnes, D.; Mason, B.; Cannell, A.; Taylor R.J.; Zomaya, A.; Millican, A.; Leonard Morphy, J.R.; Wales, M.; Perry, M.; Allen, R.A.; Gozzard, N.; Hughes, B.; Higgs, G. CDP840. A prototype of a novel class of orally active antiinflammatory phosphodiesterase 4 inhibitors. Bioorg. Med. Chem. Lett., 2002, 12, 1451-1456.

[80] Guay, D.; Hamel, P.; Blouin, M.; Brideau, C.; Chan, C.C.; Chauret, N.; Ducharme, Y.; Huang, Z.; Girard, M.; Jones, T.R; Laliberte, F.; Masson, P.; McAuliffe, M.; Piechuta, H.H.; Silva, J.; Young, R.N.; Girard, Y. Discovery of L-791,943: a potent, selective, non-emetic and orally active phosphodiesterase- 4 inhibitor. Bioorg. Med. Chem. Lett., 2002, 12, 1457-1461.

[81] Frenette, R.; Blouin, M.; Brideau, C.; Chauret, N.; Ducharme, Y. Friesen, R.W.; Hamel, P.P.; Jones, T.R.; Laliberte F.; Li, C.; Masson, P.; McAuliffe, M.; Girard, Y. Substituted 4-(2,2diphenylethyl)pyridine-N-oxides as phosphodiesterase-4 inhibitors: SAR study directed toward the improvement of pharmacokinetic parameters. Bioorg. Med. Chem. Lett., 2002, 12, 3009-3013.

[82] Haginoya, N.; Kobayashi, S.; Komoriya, S.; Yoshino, T.; Nagata, T.; Hirokawa, Y.; Nagahara, T. Design, synthesis, and biological activity of non-amidine factor Xa inhibitors containing pyridine $\mathrm{N}$ oxide and 2-carbamoylthiazole units. Bioorg. Med. Chem., 2004, 12, 5579-5586. 